\title{
Are Generalized Cut-Set Bounds Tight for the Deterministic Interference Channel?
}

\author{
Mehrdad Kiamari and A. Salman Avestimehr \\ Department of Electrical Engineering, \\ University of Southern California \\ Emails: kiamari@usc.edu and avestimehr@ee.usc.edu
}

\begin{abstract}
We propose the idea of extended networks, which is constructed by replicating the users in the two-user deterministic interference channel (DIC) and designing the interference structure among them, such that any rate that can be achieved by each user in the original network can also be achieved simultaneously by all replicas of that user in the extended network. We demonstrate that by carefully designing extended networks and applying the generalized cut-set (GCS) bound to them, we can derive a tight converse for the two-user DIC. Furthermore, we generalize our techniques to the three-user DIC, and demonstrate that the proposed approach also results in deriving a tight converse for the three-user DIC in the symmetric case.
\end{abstract}

Index Terms-Deterministic Interference Channel, Generalized Cut-Set Bound, Converse.

\section{INTRODUCTION}

A common tool for deriving outer bounds on the capacity region of communication networks is the cut-set bound [1]. It is well-known that the cut-set bound is tight in several general problems, such as the unicast and multicast wireline networks [2]-[3]. Furthermore, it can also approximate the capacity of Gaussian relay networks to within a constant gap [4]. However, once we go beyond the unicast and multicast problems, the cut-set bound is typically loose, even for the simplest case of the two-user deterministic interference channel (DIC) [5]. Therefore, to make progress in these problems, researchers have focused on obtaining new techniques to develop tighter outer bounds, such as the genie-aided bounds (see, e.g., [6]). Although these techniques enable progress, they are typically tailored to the specific problem setting that is considered, and unlike the cut-set bound they cannot be systematically applied to other problems.

As a result, there have recently been several efforts to generalize the cut-set bound in order to systematically obtain tighter outer bounds on the capacity region of communication networks, in particular the generalized network sharing (GNS) bound for wireline networks [7], the generalized cut-set (GCS) bound for deterministic networks [8], and its extension to noisy networks [9]. The key idea behind the GCS bound [8], which is the main focus of this paper, is to construct a serial concatenation of a network and derive a tighter bound by applying the cut-set bound to the concatenated network, with a special restriction on its allowed transmit signal distributions.
This bound has resulted in development of several new outer bounds on the deterministic $k$-unicast two-hop networks [8].

In this paper, we focus on the two-user DIC and investigate whether GCS bound can be systematically utilized for deriving a tight converse for this problem. While directly applying GCS bound on the two-user DIC does not lead to tight outer bounds, we introduce the idea of extended networks, and show that, quite interestingly, by carefully designing extended networks and applying the GCS bound to them, we are able to derive tight outer bounds on the capacity region of the two-user DIC. The main idea behind extended networks is to create several copied versions of each user in the two-user DIC, with an interference structure among them, such that all copied users in the extended network can simultaneously achieve the same rate as the original user by deploying the same coding scheme as the one used in the original network. Therefore, any sum-rate bound on the extended network can lead to a weighted sum-rate bound on the original network, where the coefficient of the corresponding rate weighted sum-rate bound depends on the number of copied versions of each user in the extended network. We demonstrate that by carefully designing the structure of extended networks and applying GCS bound to them, we can systematically derive all outer bounds not the capacity region of the two-user DIC.

The idea of extended networks is general, and can also be utilized to derive outer bounds on interference channels with more than two users. We consider the symmetric threeuser DIC [10], and demonstrate that all outer bounds on the capacity region of this problem can also be recovered by carefully constructing the corresponding extended network for each outer bounds and applying the GCS to it.

We start the paper by describing the system model of the two-user DIC and its capacity region in Section II, and providing an overview of the generalized cut-set (GCS) bound [8] in Section [III. We then formally introduce the idea of extended networks in Section IV] and demonstrate how all bounds on the capacity region of the two-user DIC can be derived by constructing extended networks and applying the GCS bound to them. We finally consider the three-user DIC in Section $\mathrm{V}$, and demonstrate how the ideas proposed in this paper can be generalized beyond two users to derive all outer bounds on the capacity region of this problem in the symmetric case. 


\section{System Model of the Two-User DIC}

The general system model of the two-user DIC is depicted in Fig. 1. This network, which is denoted by $\mathcal{N}$, consists of two source nodes $S_{1}$ and $S_{2}$ producing $W_{1} \in\left\{1,2, \ldots, M_{1}\right\}$ and $W_{2} \in\left\{1,2, \ldots, M_{2}\right\}$ respectively. Then, encoder 1 and encoder 2 maps their corresponding messages, i.e. $W_{1}$ and $W_{2}$, into $\mathbf{X}_{1}^{n}=\left(X_{1}[1], \ldots, X_{1}[n]\right)$ and $\mathbf{X}_{2}^{n}=\left(X_{2}[1], \ldots, X_{2}[n]\right)$ respectively. The received signals at destination 1 and destination 2 , i.e. $Y_{1}$ and $Y_{2}$ are deterministic functions of transmitted signals $X_{1}, X_{2}$, and interference signals $V_{1}$ and $V_{2}$ :

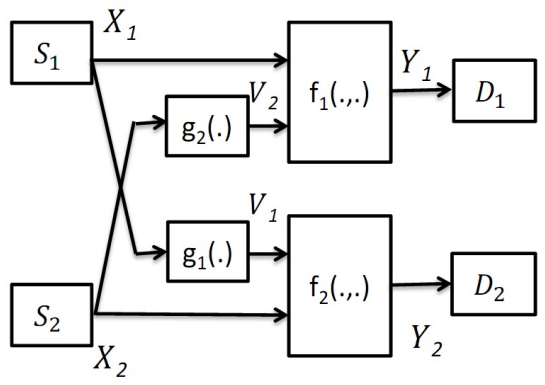

Fig. 1. System model of the two-user DIC.

$$
\begin{aligned}
& Y_{1}=f_{1}\left(X_{1}, V_{2}\right) \\
& Y_{2}=f_{2}\left(X_{2}, V_{1}\right) \\
& V_{1}=g_{1}\left(X_{1}\right) \\
& V_{2}=g_{2}\left(X_{2}\right)
\end{aligned}
$$

where $g_{1}($.$) and g_{2}($.$) are not generally invertible functions$ and $f_{1}(.,$.$) and f_{2}(.,$.$) satisfy the following conditions which$ is the same as [5]

$$
\begin{aligned}
& H\left(Y_{1} \mid X_{1}\right)=H\left(V_{2}\right) \\
& H\left(Y_{2} \mid X_{2}\right)=H\left(V_{1}\right)
\end{aligned}
$$

these conditions are equivalent to

$$
\begin{aligned}
& V_{1}=h_{2}\left(X_{2}, Y_{2}\right) \\
& V_{2}=h_{1}\left(X_{1}, Y_{1}\right)
\end{aligned}
$$

Theorem 1. The capacity region $C$ of the two-user DIC is the union of all set of rate tuple $\left(R_{1}, R_{2}\right)$ satisfying

$$
\begin{aligned}
R_{1} & \leq H\left(Y_{1} \mid V_{2}\right) \\
R_{2} & \leq H\left(Y_{2} \mid V_{1}\right) \\
R_{1}+R_{2} & \leq H\left(Y_{1}\right)+H\left(Y_{2} \mid V_{1}, V_{2}\right) \\
R_{1}+R_{2} & \leq H\left(Y_{2}\right)+H\left(Y_{1} \mid V_{1}, V_{2}\right) \\
R_{1}+R_{2} & \leq H\left(Y_{1} \mid V_{1}\right)+H\left(Y_{2} \mid V_{2}\right) \\
2 R_{1}+R_{2} & \leq H\left(Y_{1}\right)+H\left(Y_{2} \mid V_{2}\right)+H\left(Y_{1} \mid V_{1}, V_{2}\right) \\
2 R_{2}+R_{1} & \leq H\left(Y_{2}\right)+H\left(Y_{1} \mid V_{1}\right)+H\left(Y_{2} \mid V_{1}, V_{2}\right)
\end{aligned}
$$

over all product probability distributions $p\left(x_{1}\right) p\left(x_{2}\right)$.

Proof: The achievable scheme for this problem is based on Han-Kobayashi (HK) [11], and the converse has been derived by El Gamal and Costa [5].

As it is mentioned in introduction, our goal is to demonstrate all outer bounds (4a)-(4g) can be systematically derived by the idea of extended network and applying GCS bound to it. To that end, we next introduce GCS bound and extended networks. A simple version of extended networks idea, called side-way concatenation, was first introduced in [12]. However, with this side-way concatenation, one can only recover bounds (4f) and (4g)(See section 7.3.1 of [12]). The main contribution of this paper is to demonstrate that all bounds (4a)-(4f) can be recovered by the idea of extended networks and applying the GCS bound to them. Not only can this technique be utilized for the two-user DIC, but it can be applied for three-user DIC as well.

\section{Overview of Generalized Cut Set Bound}

Generalized Cut-Set bound is a generalization of classical cut-set bound introduced by [8] which allows for deriving upper bound on capacity region.

In particular, consider a K-unicast memoryless network $\mathcal{N}$ including a set of nodes $\mathcal{V}$ which has $K$ sources $\mathcal{S}=$ $\left[S_{1}, \ldots, S_{K}\right]$ and $K$ destinations $\mathcal{D}=\left[D_{1}, \ldots, D_{K}\right]$. At each time step $t, X_{v}[t]$ is the transmitted symbol from $v \in \mathcal{V}$ and $Y_{v}[t]$ is the received symbol at $v \in \mathcal{V}$. In a deterministic network, the received signals at time step $t$ is a function of transmitted signals which means $Y_{\mathcal{V}}[t]=F\left(X_{\mathcal{V}}[t]\right)$. We assume that source nodes do not receive any symbols and destination nodes do not transmit any symbols.

The coding scheme $\mathcal{C}_{n}$ with rate tuple $\left(R_{1}, \ldots, R_{K}\right)$ can be expressed as follow

1) Encoder $E_{i}:\left\{1,2, \ldots, 2^{n R_{i}}\right\} \longrightarrow \mathcal{X}_{S_{i}}^{n}$ for $i=1, \ldots, K$

2) Relaying node $r_{v}^{(t)}: r_{v}^{(t-1)} \longrightarrow \mathcal{X}_{v}$ for $v \in \mathcal{V} \backslash\{\mathcal{S} \cup \mathcal{D}\}$ and $t=1, \ldots, n$

3) Decoder $D_{i}: \mathcal{Y}_{D_{i}}^{n} \longrightarrow\left\{1,2, \ldots, 2^{n R_{i}}\right\}$ for $i=1, \ldots, K$ and error probability $P_{e}\left(\mathcal{C}_{n}\right)$ can be written as

$$
\begin{gathered}
P_{e}\left(\mathcal{C}_{n}\right)=\operatorname{Prob}\left\{W_{i} \neq g_{i}\left(Y_{D_{i}}[1], \ldots, Y_{D_{i}}[n]\right)\right. \\
\text { for some } i \in 1, \ldots, K\} .
\end{gathered}
$$

A rate tuple $\left(R_{1}, \ldots, R_{K}\right)$ is said to be achievable if for any $\epsilon>0$, there exists a coding scheme such that $P_{e}\left(\mathcal{C}_{n}\right) \leq \epsilon$ for some large $n$. The closure of all achievable rate tuples would specify the capacity region $C \subset \mathbb{R}_{+}^{K}$. [8] has derived an upper bound for sum-rate on $\mathcal{N}$ in the case of deterministic networks as follow

Theorem 2 ([8]). If a rate tuple $\left(R_{1}, \ldots, R_{K}\right)$ is achievable on a $K$-unicast deterministic network, then it can be shown that there exists a joint distribution $p\left(x_{V}\right)$ on sources such that

$$
\sum_{i=1}^{K} R_{i} \leq \sum_{j=1}^{l} I\left(X_{\Omega_{j}} ; Y_{\Omega_{j}^{c}} \mid X_{\Omega_{j}^{c}}, Y_{\Omega_{j-1}^{c}}\right)
$$

for all choices of $l$ node subsets $\Omega_{1}, \ldots ., \Omega_{l}$ such that $\mathcal{V}=$ $\Omega_{0} \supseteq \Omega_{1} \supseteq \ldots \supseteq \Omega_{l+1}=\emptyset$, and $d_{i} \in \Omega_{j} \Longleftrightarrow s_{i} \in \Omega_{j+1}$ for $i=1, \ldots, K$ and $j=0, \ldots, l$.

It should be noted that in the case of deterministic network, (6) can be written as follow

$$
\sum_{i=1}^{K} R_{i} \leq \sum_{j=1}^{l} H\left(Y_{\Omega_{j}^{c}} \mid X_{\Omega_{j}^{c}}, Y_{\Omega_{j-1}^{c}}\right) .
$$

As an example of GCS bound, we demonstrate that (4c) and (4d) can be derived by applying Theorem 2 on the two-user 
DIC. It is easy to verify that (4c) and (4d) cannot be found through classical cut-set bounds. If a rate tuple $\left(R_{1}, R_{2}\right)$ is achievable on $\mathcal{N}$, then there exists a joint distribution on the sources of $\mathcal{N}$ such that

$$
\begin{aligned}
& R_{1}+R_{2} \leq H\left(Y_{1}\right)+H\left(Y_{2} \mid V_{1}, V_{2}\right) \\
& R_{1}+R_{2} \leq H\left(Y_{2}\right)+H\left(Y_{1} \mid V_{1}, V_{2}\right)
\end{aligned}
$$

By utilizing GCS bound on the original network with the cuts depicted in Fig. 2, we have

$$
\begin{aligned}
R_{1}+R_{2} & \leq H\left(Y_{2}\right)+H\left(Y_{1} \mid X_{2}, Y_{2}\right) \\
& =H\left(Y_{2}\right)+H\left(Y_{1} \mid X_{2}, Y_{2}, g_{2}\left(X_{2}\right), h_{2}\left(X_{2}, Y_{2}\right)\right) \\
& =H\left(Y_{2}\right)+H\left(Y_{1} \mid X_{2}, Y_{2}, V_{2}, V_{1}\right) \\
& \leq H\left(Y_{2}\right)+H\left(Y_{1} \mid V_{1}, V_{2}\right) .
\end{aligned}
$$

Regarding bound (4d), it suffices to consider the original network. By applying GCS bound on the original network with the cuts depicted in Fig. 3, we have

$$
\begin{aligned}
R_{1}+R_{2} & \leq H\left(Y_{1}\right)+H\left(Y_{2} \mid X_{1}, Y_{1}\right) \\
& =H\left(Y_{1}\right)+H\left(Y_{2} \mid X_{1}, Y_{1}, g_{1}\left(X_{1}\right), h_{1}\left(X_{1}, Y_{1}\right)\right) \\
& \leq H\left(Y_{1}\right)+H\left(Y_{2} \mid V_{1}, V_{2}\right) .
\end{aligned}
$$

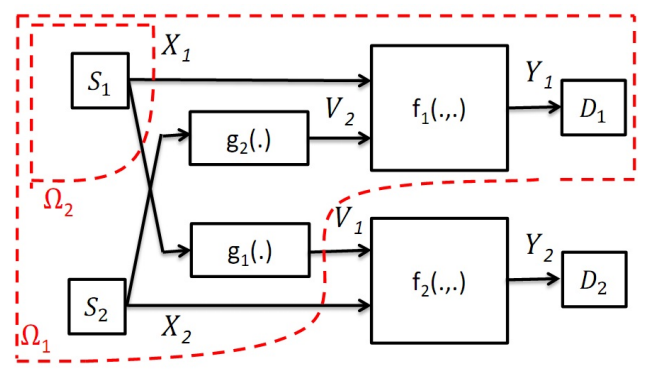

Fig. 2. Depicting the cuts for deriving bound (4c) of the two-user DIC

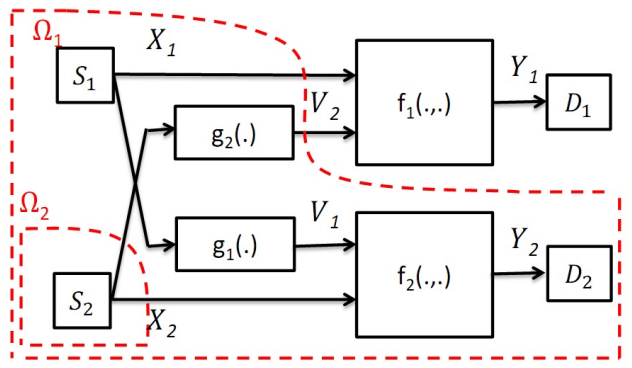

Fig. 3. Depicting the cuts for deriving bound (4d) of the two-user DIC

Although GCS method recovers two bounds, i.e. (4c)-(4d), it fails to recover the remaining five bounds. The question raised here is that whether we can obtain the rest bounds or not. We will show that performing GCS on extended network rather than the original network is a solution for finding the remaining bounds.

\section{CONVERSE FOR TWO-USER DIC}

We start by formally defining an extended network.
Definition Consider a two-user DIC, denoted by $\mathcal{N}$, as defined in section II. A $\left(k_{1}, k_{2}\right)$ extended version of $\mathcal{N}$, denoted by $N_{k_{1}, k_{2}}$ is constructed by considering $k_{1}$ and $k_{2}$ copied versions of user 1 and user 2, respectively, as depicted in Fig. 4 In this extended network, each receiver gets interference only from one of the copied versions of the other user. It should be noted that interference function, i.e. $g_{i}($.$) , and$ function $f_{i}(, .$,$) for the copied versions of user i$ are the same as the functions considered for the original user $i$ on the two-user DIC. The choice of interference pattern depends on the outer bound which is supposed to be derived. In Fig. 4. $S_{i}^{(j)}$ represents the source node of $j$ th copied version of $i$ th user. The message corresponded to source $S_{i(j)}$ is denoted by $W_{i(j)} \in\left[1: 2^{n R_{i}}\right]$. It should be noted that the messages of different sources are independent from each other even if the same coding scheme is utilized for them. In this extended network, encoder function $E_{i}:\left[1: 2^{n R_{i}}\right] \rightarrow \mathcal{X}_{S_{i}}^{n}$ and decoder function $D_{i}: \mathcal{Y}_{D_{i}}^{n} \rightarrow\left[1: 2^{n R_{i}}\right]$ for $i=1,2$ are applied to the copied versions of $i$ th user. Then, encoder function $E_{i}$ maps the corresponding message $W_{i(j)}$ into $X_{i(j)}^{n}=$ $\left[X_{i^{(j)}}[1], \ldots, X_{i^{(j)}}[n]\right]$. Furthermore, the received signal at each copy is a deterministic function of transmitted signal of its corresponding source and interference signal coming from the copied versions of the other user.

Lemma 1. If a rate tuple $\left(R_{1}, R_{2}\right)$ is achievable on the two-user DIC, $\mathcal{N}$, then rate tuple $\{\underbrace{R_{1}, \ldots, R_{1}}_{k_{1}}, \underbrace{R_{2}, \ldots, R_{2}}_{k_{2}}\}$ is achievable on any $\left(k_{1}, k_{2}\right)$ extended version of the network, $N_{k_{1}, k_{2}}$ by applying the same coding scheme as $S_{i}$ in $\mathcal{N}(i=$ $1,2)$ to all $S_{i}^{(j)}$ 's $\left(i=1,2, j=1, \ldots, k_{i}\right)$ in $\mathcal{N}_{k_{1}, k_{2}}$.

Proof: Consider a coding scheme that results in transmission of $X_{1}^{n}, X_{2}^{n}$ and reception of $Y_{1}^{n}, Y_{2}^{n}$ in $\mathcal{N}$. Consider a $\left(k_{1}, k_{2}\right)$ extended version of the network, $\mathcal{N}_{k_{1}, k_{2}}$, and assume the same coding scheme as $S_{i}$ in $\mathcal{N}(i=1,2)$ is applied to all $S_{i}^{(j)}$ 's $\left(i=1,2, j=1, \ldots, k_{i}\right)$ in $\mathcal{N}_{k_{1}, k_{2}}$. Now, note the following three points;

1) The coding scheme utilized on the $j$ th copied version of user $i$ on the extended network is the same as the coding scheme used on user $i$ on the original network

2) The interference function, i.e. $g_{i}($.$) , and function f_{i}(.,$. in the extended network are the same as the functions considered for user $i$ in the original network

3 ) user $i$ gets interference only from one of copied versions of user $\bar{i}$ for $i=1,2(\bar{i}=3-i)$

It is easy to see that these three points imply $I\left(X_{i^{(j)}}^{n}, Y_{i^{(j)}}^{n}\right)=$ $I\left(X_{i}^{n}, Y_{i}^{n}\right) \quad \forall j=1, \ldots, k_{i}, i=1,2$. Therefore, any rate tuple $\{\underbrace{R_{1}, \ldots, R_{1}}_{k_{1}}, \underbrace{R_{2}, \ldots, R_{2}}_{k_{2}}\}$ is achievable on any $\left(k_{1}, k_{2}\right)$ extended version of the network, $N_{k_{1}, k_{2}}$.

Therefore, any sum-rate upper bound on $\mathcal{N}_{k_{1}, k_{2}}$ would result in an upper bound on $k_{1} R_{1}+k_{2} R_{2}$ in the original network. In particular, by applying GCS bound on $N_{k_{1}, k_{2}}$, we can systematically derive a bound for $k_{1} R_{1}+k_{2} R_{2}$ on $\mathcal{N}$. 
In the remaining parts, we derive all remaining outer bounds, i.e. (4a),(4b),(4e),(4f), and (4g), by designing specific extended network for each of them and applying GCS to them.

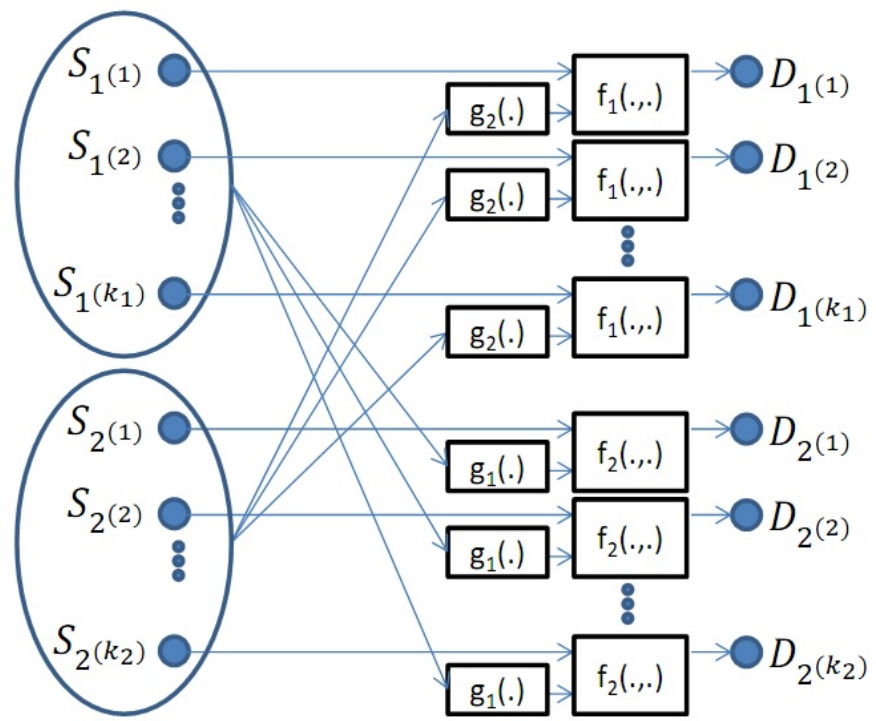

Fig. 4. System model of the extended network $\mathcal{N}_{k_{1}, k_{2}}$.

\section{A. Deriving Bounds (4a) and (4b)}

In this section, we demonstrate how to derive individual rate bound (4a) by applying GCS bound to an appropriate extended network. Due to symmetry, we only need to find the bound for user 1, i.e. (4a).

We utilize extended network $\mathcal{N}_{k, 1}$, as depicted in Fig. 5 for this bound. As you can note, in this network, second user imposes interference on all copied versions of first user.

According to Lemma 1 , if a rate tuple $\left(R_{1}, R_{2}\right)$ is achievable on $\mathcal{N}$, then rate tuple $(\underbrace{R_{1}, \ldots, R_{1}}_{k}, R_{2})$ is achievable on $\mathcal{N}_{k, 1}$.

Consider $\left\{\mathcal{C}_{n}\right\}$ as a sequence of coding scheme with block length $n$ that achieves sum rate $R_{\Sigma}$ on $\mathcal{N}_{k, 1}$. Therefore, by applying GCS bound on the extended network $\mathcal{N}_{k, 1}$ and picking cuts $\Omega_{1}, \ldots, \Omega_{k+1}$ depicted in Fig. 5. we have

$$
\begin{aligned}
n R_{\Sigma} & =n\left(R_{1}+R_{2}+(k-1) R_{1}\right) \\
& \stackrel{(a)}{\leq} I\left(W_{\mathcal{S}} ; Y_{\Omega_{1}^{c}}^{n}\right) \\
& +\sum_{l=2}^{k+1} I\left(W_{\mathcal{S} \cap \Omega_{l}} ; Y_{\Omega_{l}^{c}}^{n} \cap \Omega_{l-1} \mid W_{\mathcal{S} \backslash \Omega_{l}}, Y_{\Omega_{l-1}^{c}}^{n}\right)+n \epsilon_{n} \\
& \stackrel{(b)}{=} H\left(Y_{\Omega_{1}^{c}}^{n}\right)+\sum_{l=2}^{k+1} H\left(Y_{\Omega_{l}^{c}}^{n} \cap \Omega_{l-1} \mid W_{\mathcal{S} \backslash \Omega_{l}}, Y_{\Omega_{l-1}^{c}}^{n}\right)+n \epsilon_{n} \\
& \stackrel{(c)}{\leq} \sum_{i=1}^{n} H\left(Y_{1^{(1)}}[i]\right)+(k-1) \sum_{i=1}^{n} H\left(Y_{1^{(1)}}[i] \mid V_{2^{(1)}}[i]\right) \\
& +\sum_{i=1}^{n} H\left(Y_{2^{(1)}}[i] \mid V_{2^{(1)}}[i], V_{1^{(1)}}[i]\right)+n \epsilon_{n}
\end{aligned}
$$

where $(a)$ follows from Theorem 1 of [8] (in particular, inequality (6) in [8] in the proof of Theorem 1) on the extended network shown in Fig. 5. Step (b) follows from considering deterministic model. Finally, step $(c)$ follows from proof the presented in Appendix A.

By letting $k$ goes to infinity, we have

$$
n\left(R_{1}\right) \leq \sum_{i=1}^{n} H\left(Y_{1^{(1)}}[i] \mid V_{2^{(1)}}[i]\right)+n \epsilon_{n}
$$

which would result in bound (4a).

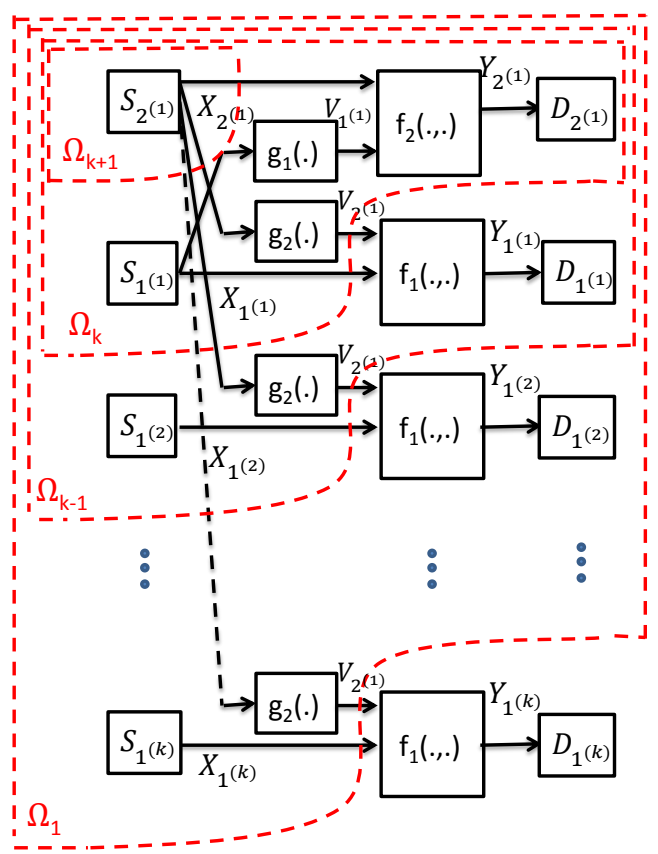

Fig. 5. Extended network and the cuts for deriving bound (4a) of the two-user DIC

\section{B. Deriving Bound (4e)}

In this section, we design the extended network $\mathcal{N}_{k, k}$ illustrated in Fig. 6 to derive bound (4e). Based on Lemma [1. we know that it a rate tuple $\left(R_{1}, R_{2}\right)$ is achievable on $\mathcal{N}$, then rate tuple $(\underbrace{R_{1}, \ldots, R_{1}}_{k}, \underbrace{R_{2}, \ldots, R_{2}})$ is achievable on $\mathcal{N}_{k, k}$. Assume $\left\{\mathcal{C}_{n}\right\}$ as a sequence of coding scheme with block length $n$ that achieves sum rate $R_{\Sigma}$ on $\mathcal{N}_{k, k}$. Therefore, by applying GCS bound on the extended network $\mathcal{N}_{k, k}$ and picking cuts $\Omega_{1}, \ldots, \Omega_{2 k}$ depicted in Fig. 6, we have

$$
\begin{aligned}
n R_{\Sigma} & =n\left(R_{1}+R_{2}+(k-1)\left(R_{1}+R_{2}\right)\right) \\
& \stackrel{(a)}{\leq} I\left(W_{\mathcal{S}} ; Y_{\Omega_{1}^{c}}^{n}\right) \\
& +\sum_{l=2}^{2 k} I\left(W_{\mathcal{S} \cap \Omega_{l}} ; Y_{\Omega_{l}^{c}}^{n} \cap \Omega_{l-1} \mid W_{\mathcal{S} \backslash \Omega_{l}}, Y_{\Omega_{l-1}^{c}}^{n}\right)+n \epsilon_{n} \\
& \stackrel{(b)}{=} H\left(Y_{\Omega_{1}^{c}}^{n}\right)+\sum_{l=2}^{2 k} H\left(Y_{\Omega_{l}^{c} \cap \Omega_{l-1}}^{n} \mid W_{\mathcal{S} \backslash \Omega_{l}}, Y_{\Omega_{l-1}^{c}}^{n}\right)+n \epsilon_{n}
\end{aligned}
$$




$$
\begin{aligned}
& \stackrel{(c)}{\leq} \sum_{i=1}^{n} H\left(Y_{2^{(1)}}[i]\right) \\
& \quad+k \sum_{i=1}^{n} H\left(Y_{1^{(1)}}[i] \mid V_{1^{(1)}}[i]\right) \\
& \quad+(k-1) \sum_{i=1}^{n} H\left(Y_{2^{(1)}}[i] \mid V_{2^{(1)}}[i]\right)+n \epsilon_{n}
\end{aligned}
$$

where $(a)$ follows from Theorem 1 of [8] (in particular, inequality (6) in [8]) on the extended network depicted in Fig. 6. Step (b) follows from considering deterministic model. Finally, step $(c)$ follow from the proof presented in Appendix A.

As $k$ goes to infinity, we have

$$
\begin{aligned}
n\left(R_{1}+R_{2}\right) \leq & \sum_{i=1}^{n} H\left(Y_{1^{(1)}}[i] \mid V_{1^{(1)}}[i]\right) \\
& +\sum_{i=1}^{n} H\left(Y_{2^{(1)}}[i] \mid V_{2^{(1)}}[i]\right)+n \epsilon_{n}
\end{aligned}
$$

which would result in bound (4e).

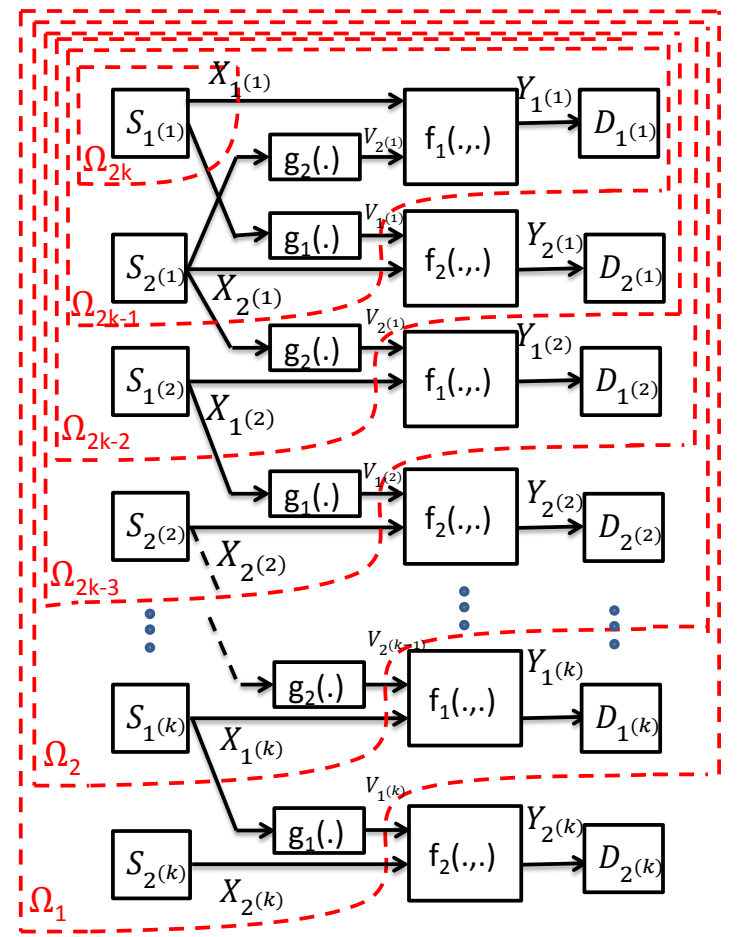

Fig. 6. Extended network and the cuts for deriving bound (4e) of the two-user DIC

\section{Deriving Bounds $(4 f)$ and $(4 g)$}

In this part, the extended network $\mathcal{N}_{2,1}$, depicted in Fig. 7. is utilized to derive bound (4f). By applying GCS bound on this extended network and picking cuts $\Omega_{1}, \Omega_{2}$ and $\Omega_{3}$ depicted in Fig. 7, we have

$$
\begin{aligned}
& n R_{\Sigma}=n\left(R_{1}+R_{2}+R_{1}\right) \\
& \stackrel{(a)}{\leq} I\left(W_{\mathcal{S}} ; Y_{\Omega_{1}^{c}}^{n}\right)+I\left(W_{\mathcal{S} \cap \Omega_{2}} ; Y_{\Omega_{2}^{c}}^{n} \cap \Omega_{1} \mid W_{\mathcal{S} \backslash \Omega_{2}}, Y_{\Omega_{1}^{c}}^{n}\right) \\
& +I\left(W_{\mathcal{S} \cap \Omega_{3}} ; Y_{\Omega_{3}^{c}}^{n} \cap \Omega_{2} \mid W_{\mathcal{S} \backslash \Omega_{3}}, Y_{\Omega_{2}^{c}}^{n}\right)+n \epsilon_{n} \\
& \stackrel{(b)}{=} H\left(Y_{\Omega_{1}^{c}}^{n}\right)+H\left(Y_{\Omega_{2}^{c}}^{n} \cap \Omega_{1} \mid W_{\mathcal{S} \backslash \Omega_{2}}, Y_{\Omega_{1}^{c}}^{n}\right) \\
& +H\left(Y_{\Omega_{3}^{c} \cap \Omega_{2}}^{n} \mid W_{\mathcal{S} \backslash \Omega_{3}}, Y_{\Omega_{2}^{c}}^{n}\right)+n \epsilon_{n} \\
& \stackrel{(c)}{\leq} \sum_{i=1}^{n} H\left(Y_{1^{(1)}}[i]\right)+\sum_{i=1}^{n} H\left(Y_{2^{(1)}}[i] \mid V_{2^{(1)}}[i]\right) \\
& +\sum_{i=1}^{n} H\left(Y_{1^{(1)}}[i] \mid V_{2^{(1)}}[i], V_{1^{(1)}}[i]\right)+n \epsilon_{n}
\end{aligned}
$$

where $(a)$ follows from Theorem 1 of [8] (in particular, inequality (6) in [8]) on the extended network depicted in Fig. 7 Step $(b)$ follows from considering deterministic model. Finally, step $(c)$ follow from the proof presented in Appendix A.

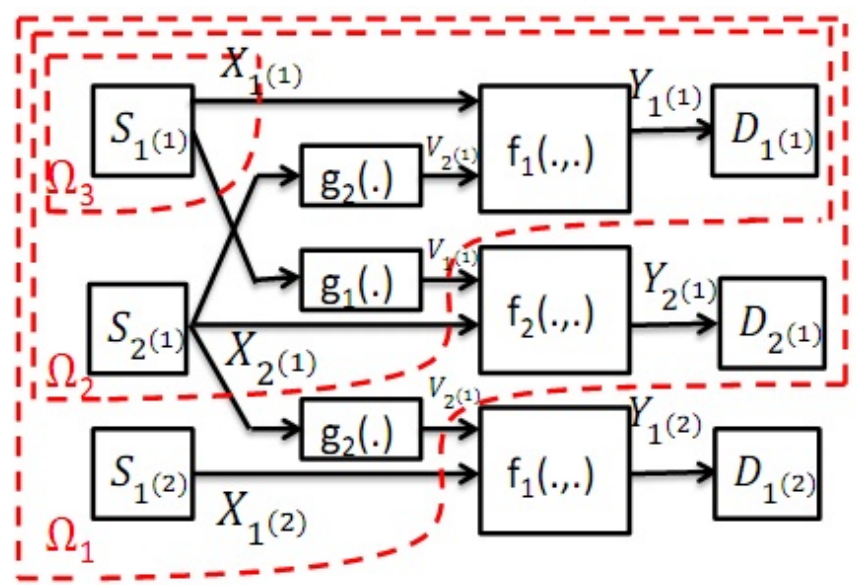

Fig. 7. Extended network and the cuts for deriving bound (4f) of the two-user DIC

Bound ( $4 \mathrm{~g}$ ) can be derived by considering its corresponding extended network, i.e. $\mathcal{N}_{1,2}$, and following the similar procedure.

Now, we focus on obtaining the outer bounds of the symmetric three-user DIC.

\section{Extension to the Symmetric Three-USer DIC}

In this section, we consider the symmetric three-user DIC whose capacity region is found in [10]. We demonstrate that all outer bounds can be systematically derived by the idea of the extended network and applying GCS bound to it. We start the derivation of outer bounds by defining the system model of the symmetric three-user DIC.

\section{A. System Model of the Symmetric Three-user DIC}

The system model of the symmetric three-user DIC is illustrated in Fig. 8. In this section, the outer bounds of the symmetric three-user DIC are obtained by applying GCS on different extended networks. Since the same type of choosing cuts have been utilized, the procedure of finding outer bounds 
are similar to the case of finding the outer bounds of the twouser DIC. The received signals at destination 1, destination 2, and destination 3, i.e. $Y_{1}, Y_{2}$, and $Y_{3}$ are deterministic functions of transmitted signals $X_{1}, X_{2}, X_{3}$, and interference signals $V_{1}, V_{2}$ and $V_{3}$ as follow:

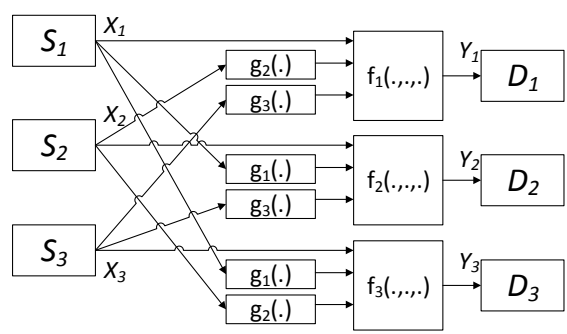

Fig. 8. System model of the symmetric three-user DIC

$$
\begin{aligned}
& Y_{1}=f_{1}\left(X_{1}, V_{2}, V_{3}\right) \\
& Y_{2}=f_{2}\left(X_{2}, V_{1}, V_{3}\right) \\
& Y_{3}=f_{3}\left(X_{3}, V_{1}, V_{2}\right) \\
& V_{1}=g_{1}\left(X_{1}\right) \\
& V_{2}=g_{2}\left(X_{2}\right) \\
& V_{3}=g_{3}\left(X_{3}\right)
\end{aligned}
$$

where $g_{1}(),. g_{2}($.$) , and g_{3}($.$) are not generally invertible$ functions and $f_{1}(.,),. f_{2}(.,$.$) , and f_{3}(.,$.$) satisfy the following$ conditions which is the same as [10]

$$
\begin{aligned}
& H\left(Y_{1} \mid X_{1}\right)=H\left(V_{2}, V_{3}\right) \\
& H\left(Y_{2} \mid X_{2}\right)=H\left(V_{1}, V_{3}\right) \\
& H\left(Y_{3} \mid X_{3}\right)=H\left(V_{2}, V_{3}\right)
\end{aligned}
$$

these conditions are equivalent to

$$
\begin{aligned}
& \left(V_{2}, V_{3}\right)=h_{1}\left(X_{1}, Y_{1}\right) \\
& \left(V_{1}, V_{3}\right)=h_{2}\left(X_{2}, Y_{2}\right) \\
& \left(V_{1}, V_{2}\right)=h_{3}\left(X_{3}, Y_{3}\right)
\end{aligned}
$$

The capacity region of the symmetric three-user DIC is characterized in [10]. It was shown by [10] that the capacity region is the union of six regions each of which is characterized by 28 linear bounds as follow

$$
\begin{gathered}
R_{1} \leq H\left(Y_{1} \mid V_{2} V_{3}\right) \\
R_{1}+R_{2} \leq H\left(Y_{1} \mid V_{1} V_{2} V_{3}\right)+H\left(Y_{2} \mid V_{3}\right) \\
R_{1}+R_{2} \leq H\left(Y_{1} \mid V_{1} V_{3}\right)+H\left(Y_{2} \mid V_{2} V_{3}\right) \\
2 R_{1}+R_{2} \leq H\left(Y_{1} \mid V_{3}\right)+H\left(Y_{1} \mid V_{1} V_{2} V_{3}\right)+H\left(Y_{2} \mid V_{2} V_{3}\right) \\
R_{1}+R_{2}+R_{3} \leq H\left(Y_{1} \mid V_{1}\right)+H\left(Y_{2} \mid V_{2} V_{3}\right)+H\left(Y_{3} \mid V_{1} V_{2} V_{3}\right) \\
R_{1}+R_{2}+R_{3} \leq H\left(Y_{1} \mid V_{1} V_{3}\right)+H\left(Y_{2} \mid V_{1} V_{2}\right)+H\left(Y_{3} \mid V_{2} V_{3}\right) \\
R_{1}+R_{2}+R_{3} \leq H\left(Y_{1} \mid V_{1} V_{2} V_{3}\right)+H\left(Y_{2} \mid V_{1}\right)+H\left(Y_{3} \mid V_{2} V_{3}\right) \\
R_{1}+R_{2}+R_{3} \leq H\left(Y_{1} \mid V_{1} V_{2} V_{3}\right)+H\left(Y_{2} \mid V_{1} V_{2} V_{3}\right)+H\left(Y_{3}\right) \\
2 R_{1}+R_{2}+R_{3} \leq H\left(Y_{1}\right)+H\left(Y_{1} \mid V_{1} V_{2} V_{3}\right)+H\left(Y_{2} \mid V_{2} V_{3}\right) \\
+H\left(Y_{3} \mid V_{1} V_{2} V_{3}\right)
\end{gathered}
$$

$$
\begin{aligned}
2 R_{1}+R_{2}+R_{3} & \leq H\left(Y_{1} \mid V_{1}\right)+H\left(Y_{1} \mid V_{1} V_{2} V_{3}\right)+H\left(Y_{2} \mid V_{2} V_{3}\right) \\
& +H\left(Y_{3} \mid V_{2} V_{3}\right) \\
2 R_{1}+R_{2}+R_{3} & \leq H\left(Y_{1} \mid V_{1} V_{3}\right)+H\left(Y_{1} \mid V_{2}\right)+H\left(Y_{2} \mid V_{1} V_{2} V_{3}\right) \\
& +H\left(Y_{3} \mid V_{2} V_{3}\right) \\
2 R_{1}+R_{2}+R_{3} & \leq H\left(Y_{1} \mid V_{1} V_{2} V_{3}\right)+H\left(Y_{1} \mid V_{3}\right)+H\left(Y_{2} \mid V_{1} V_{2}\right) \\
& +H\left(Y_{3} \mid V_{2} V_{3}\right) \\
2 R_{1}+R_{2}+R_{3} & \leq H\left(Y_{1} \mid V_{1} V_{2} V_{3}\right)+H\left(Y_{1} \mid V_{3}\right)+H\left(Y_{2} \mid V_{2}\right) \\
& +H\left(Y_{3} \mid V_{1} V_{2} V_{3}\right)
\end{aligned}
$$$$
2 R_{1}+R_{2}+R_{3} \leq H\left(Y_{1} \mid V_{1} V_{2} V_{3}\right)+H\left(Y_{1} \mid V_{1} V_{2}\right)+H\left(Y_{2} \mid V_{2} V_{3}\right)
$$$$
+H\left(Y_{3} \mid V_{3}\right)
$$$$
2 R_{1}+R_{2}+R_{3} \leq 2 H\left(Y_{1} \mid V_{1} V_{2} V_{3}\right)+H\left(Y_{2}\right)+H\left(Y_{3} \mid V_{2} V_{3}\right)
$$$$
2 R_{1}+R_{2}+R_{3} \leq 2 H\left(Y_{1} \mid V_{1} V_{2} V_{3}\right)+H\left(Y_{2} \mid V_{2}\right)+H\left(Y_{3} \mid V_{3}\right)
$$$$
3 R_{1}+R_{2}+R_{3} \leq 2 H\left(Y_{1} \mid V_{1} V_{2} V_{3}\right)+H\left(Y_{1}\right)+H\left(Y_{2} \mid V_{2} V_{3}\right)
$$$$
+H\left(Y_{3} \mid V_{2} V_{3}\right)
$$

$3 R_{1}+R_{2}+R_{3} \leq 2 H\left(Y_{1} \mid V_{1} V_{2} V_{3}\right)+H\left(Y_{1} \mid V_{2}\right)+H\left(Y_{2} \mid V_{2} V_{3}\right)$

$$
+H\left(Y_{3} \mid V_{3}\right)
$$

$$
\begin{aligned}
2 R_{1}+2 R_{2}+R_{3} & \leq H\left(Y_{1}\right)+H\left(Y_{1} \mid V_{1} V_{3}\right)+2 H\left(Y_{2} \mid V_{1} V_{2} V_{3}\right) \\
& +H\left(Y_{3} \mid V_{2} V_{3}\right)
\end{aligned}
$$

$$
2 R_{1}+2 R_{2}+R_{3} \leq H\left(Y_{1}\right)+H\left(Y_{1} \mid V_{1} V_{2} V_{3}\right)+H\left(Y_{2} \mid V_{1} V_{2} V_{3}\right)
$$$$
+H\left(Y_{2} \mid V_{2} V_{3}\right)+H\left(Y_{3} \mid V_{1} V_{3}\right)
$$

$$
\begin{aligned}
2 R_{1}+2 R_{2}+R_{3} & \leq H\left(Y_{1}\right)+H\left(Y_{1} \mid V_{1} V_{2} V_{3}\right)+2 H\left(Y_{2} \mid V_{1} V_{2} V_{3}\right) \\
& +H\left(Y_{3} \mid V_{3}\right)
\end{aligned}
$$

$2 R_{1}+2 R_{2}+R_{3} \leq H\left(Y_{1} \mid V_{1}\right)+H\left(Y_{1} \mid V_{1} V_{2} V_{3}\right)+H\left(Y_{2} \mid V_{1} V_{2} V_{3}\right)$

$$
+H\left(Y_{2} \mid V_{2} V_{3}\right)+H\left(Y_{3} \mid V_{3}\right)
$$

$2 R_{1}+2 R_{2}+R_{3} \leq 2 H\left(Y_{1} \mid V_{1} V_{3}\right)+H\left(Y_{2} \mid V_{1} V_{2} V_{3}\right)+H\left(Y_{2} \mid V_{2}\right)$

$$
+H\left(Y_{3} \mid V_{2} V_{3}\right)
$$

$3 R_{1}+2 R_{2}+R_{3} \leq 2 H\left(Y_{1} \mid V_{1} V_{2} V_{3}\right)+H\left(Y_{1}\right)+H\left(Y_{2} \mid V_{1} V_{2} V_{3}\right)$

$$
+H\left(Y_{2} \mid V_{2} V_{3}\right)+H\left(Y_{3} \mid V_{3}\right)
$$

$$
\begin{aligned}
3 R_{1}+2 R_{2}+R_{3} & \leq 2 H\left(Y_{1} \mid V_{1} V_{2} V_{3}\right)+H\left(Y_{1}\right)+2 H\left(Y_{2} \mid V_{2} V_{3}\right) \\
& +H\left(Y_{3} \mid V_{1} V_{3}\right)
\end{aligned}
$$

$3 R_{1}+2 R_{2}+R_{3} \leq 2 H\left(Y_{1} \mid V_{1} V_{2} V_{3}\right)+H\left(Y_{1} \mid V_{1}\right)+2 H\left(Y_{2} \mid V_{2} V_{3}\right)$ $+H\left(Y_{3} \mid V_{3}\right)$

$$
\begin{aligned}
3 R_{1}+2 R_{2}+R_{3} & \leq 3 H\left(Y_{1} \mid V_{1} V_{2} V_{3}\right)+H\left(Y_{2} \mid V_{2} V_{3}\right)+H\left(Y_{2}\right) \\
& +H\left(Y_{3} \mid V_{3}\right)
\end{aligned}
$$

$$
\begin{aligned}
4 R_{1}+2 R_{2}+R_{3} & \leq 3 H\left(Y_{1} \mid V_{1} V_{2} V_{3}\right)+H\left(Y_{1}\right)+2 H\left(Y_{2} \mid V_{2} V_{3}\right) \\
& +H\left(Y_{3} \mid V_{3}\right)
\end{aligned}
$$

In the rest of this section, we prove bounds 19 - 46 by constructing appropriate extended networks and applying GCS on them. 
Derivation of bound (19). To derive this bound, we design the extended network considered in Fig. 9. By applying GCS bound and picking cuts depicted in Fig. 9, we have

$$
\begin{aligned}
n R_{\Sigma} & =n\left(R_{1}+R_{2}+R_{3}+k R_{1}\right) \\
& \stackrel{(a)}{\leq} H\left(Y_{\Omega_{1}^{c}}^{n}\right)+\sum_{l=2}^{k+2} H\left(Y_{\Omega_{l}^{c}}^{n} \cap \Omega_{l-1} \mid W_{\mathcal{S} \backslash \Omega_{l}}, Y_{\Omega_{l-1}^{c}}^{n}\right)+n \epsilon_{n} \\
& \stackrel{(b)}{\leq} \sum_{i=1}^{n} k H\left(Y_{1}[i] \mid V_{2}[i] V_{3}[i]\right)+C+n \epsilon_{n}
\end{aligned}
$$

where $C \triangleq \sum_{i=1}^{n} H\left(Y_{1}[i]\right)+H\left(Y_{2}[i]\right)+H\left(Y_{3}[i]\right)$ and step (a) follows from GCS [8] for deterministic networks. Step (b) follows from the proof presented in Appendix B.

By letting $k$ goes to infinity, bound (19) will be recovered.

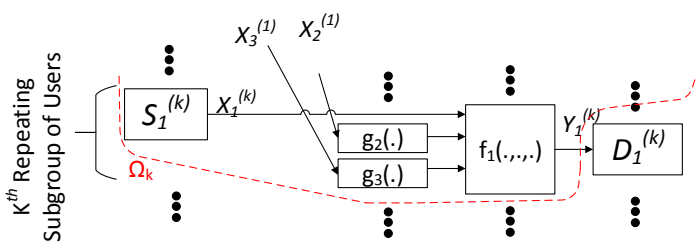

Fig. 9. Extended network and the cuts for deriving bound 19 for the three-user DIC

Derivation of bound (20). To derive this bound, we design the extended network considered in Fig. 10 By applying GCS bound and picking cuts depicted in Fig. 10, we have

$$
\begin{aligned}
n R_{\Sigma} & =n\left(R_{1}+R_{2}+R_{3}+k R_{1}+k R_{2}\right) \\
& \stackrel{(a)}{\leq} H\left(Y_{\Omega_{1}^{c}}^{n}\right)+\sum_{l=2}^{2 k+2} H\left(Y_{\Omega_{l}^{c}}^{n} \cap \Omega_{l-1} \mid W_{\mathcal{S} \backslash \Omega_{l}}, Y_{\Omega_{l-1}^{c}}^{n}\right)+n \epsilon_{n} \\
& \stackrel{(b)}{\leq} \sum_{i=1}^{n} k H\left(Y_{1}[i] \mid V_{1}[i] V_{2}[i] V_{3}[i]\right)+k H\left(Y_{2}[i] \mid V_{3}[i]\right) \\
& +C+n \epsilon_{n}
\end{aligned}
$$

where $(a)$ follows from GCS [8] for deterministic networks and step $(b)$ follows from the proof presented in Appendix B.

By letting $k$ goes to infinity, bound (20) will be recovered.

Derivation of bound (21). To derive this bound, we design the extended network considered in Fig. 11. By applying GCS bound and picking cuts depicted in Fig. II], we have

$$
\begin{aligned}
n R_{\Sigma} & =n\left(R_{1}+R_{2}+R_{3}+k R_{1}+k R_{2}\right) \\
& \stackrel{(a)}{\leq} H\left(Y_{\Omega_{1}^{c}}^{n}\right)+\sum_{l=2}^{2 k+2} H\left(Y_{\Omega_{l}^{c}}^{n} \cap \Omega_{l-1} \mid W_{\mathcal{S} \backslash \Omega_{l}}, Y_{\Omega_{l-1}^{c}}^{n}\right)+n \epsilon_{n} \\
& \stackrel{(b)}{\leq} \sum_{i=1}^{n} k H\left(Y_{1}[i] \mid V_{1}[i] V_{3}[i]\right)+k H\left(Y_{2}[i] \mid V_{2}[i] V_{3}[i]\right) \\
& +C+n \epsilon_{n}
\end{aligned}
$$

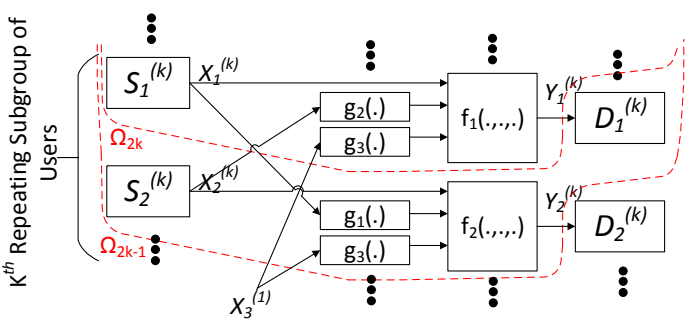

Fig. 10. Extended network and the cuts for deriving bound 20 for the three-user DIC

where $(a)$ follows from GCS [8] for deterministic networks and step $(b)$ follows from the proof presented in Appendix B.

By letting $k$ goes to infinity, bound 21] will be recovered.

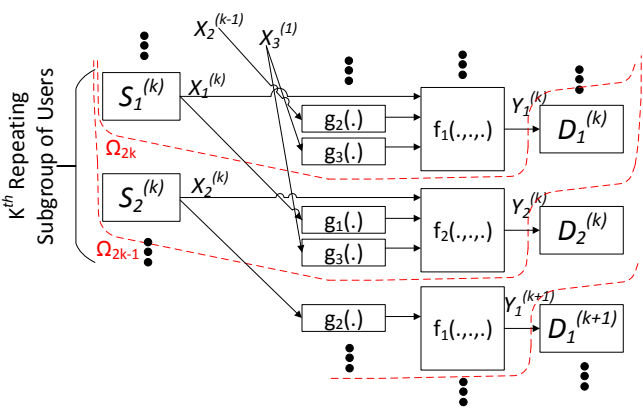

Fig. 11. Extended network and the cuts for deriving bound 21 for the three-user DIC

Derivation of bound (22). To derive this bound, we design the extended network considered in Fig. 12, By applying GCS bound and picking cuts depicted in Fig. 12, we have

$$
\begin{aligned}
n R_{\Sigma} & =n\left(R_{1}+R_{2}+R_{3}+2 k R_{1}+k R_{2}\right) \\
& \stackrel{(a)}{\leq} H\left(Y_{\Omega_{1}^{c}}^{n}\right)+\sum_{l=2}^{3 k+2} H\left(Y_{\Omega_{l}^{c}}^{n} \cap \Omega_{l-1} \mid W_{\mathcal{S} \backslash \Omega_{l}}, Y_{\Omega_{l-1}}^{n}\right)+n \epsilon_{n} \\
& \stackrel{(b)}{\leq} \sum_{i=1}^{n} k H\left(Y_{1}[i] \mid V_{3}[i]\right)+k H\left(Y_{1}[i] \mid V_{1}[i] V_{2}[i] V_{3}[i]\right) \\
& +\sum_{i=1}^{n} k H\left(Y_{2}[i] \mid V_{2}[i] V_{3}[i]\right) \\
& +C+n \epsilon_{n}
\end{aligned}
$$

where $(a)$ follows from GCS [8] for deterministic networks and step $(b)$ follows from the proof presented in Appendix B.

By letting $k$ goes to infinity, bound (22) will be recovered. Derivation of bound (23). To derive this bound, we design the extended network considered in Fig. 13. By applying GCS 


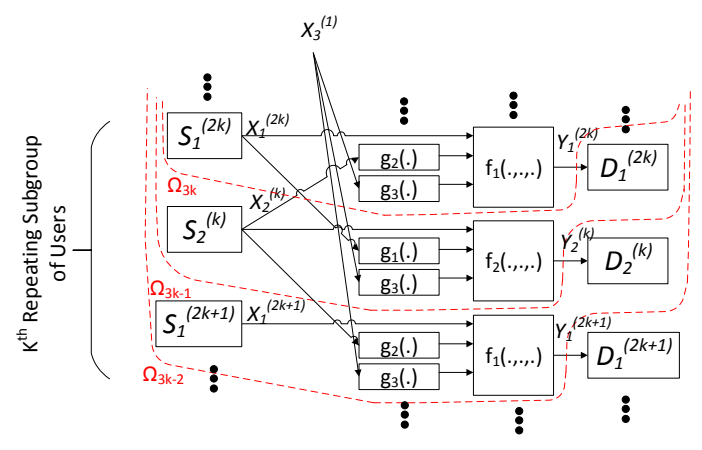

Fig. 12. Extended network and the cuts for deriving bound 22 for the three-user DIC

bound and picking cuts depicted in Fig. 13, we have

$$
\begin{aligned}
n R_{\Sigma} & =n\left(R_{1}+R_{2}+R_{3}+k R_{1}+k R_{2}+k R_{3}\right) \\
& \stackrel{(a)}{\leq} H\left(Y_{\Omega_{1}^{c}}^{n}\right)+\sum_{l=2}^{3 k+2} H\left(Y_{\Omega_{l}^{c} \cap \Omega_{l-1}}^{n} \mid W_{\mathcal{S} \backslash \Omega_{l}}, Y_{\Omega_{l-1}^{c}}^{n}\right)+n \epsilon_{n} \\
& \stackrel{(b)}{\leq} \sum_{i=1}^{n} k H\left(Y_{1}[i] \mid V_{1}[i]\right)+k H\left(Y_{2}[i] \mid V_{2}[i] V_{3}[i]\right) \\
& +\sum_{i=1}^{n} k H\left(Y_{3}[i] \mid V_{1}[i] V_{2}[i] V_{3}[i]\right) \\
& +C+n \epsilon_{n}
\end{aligned}
$$

where $(a)$ follows from GCS [8] for deterministic networks and step $(b)$ follows from the proof presented in Appendix B.

By letting $k$ goes to infinity, bound $(23)$ will be recovered. Derivation of bound (24). To derive this bound, we design the extended network considered in Fig. 14. By applying GCS

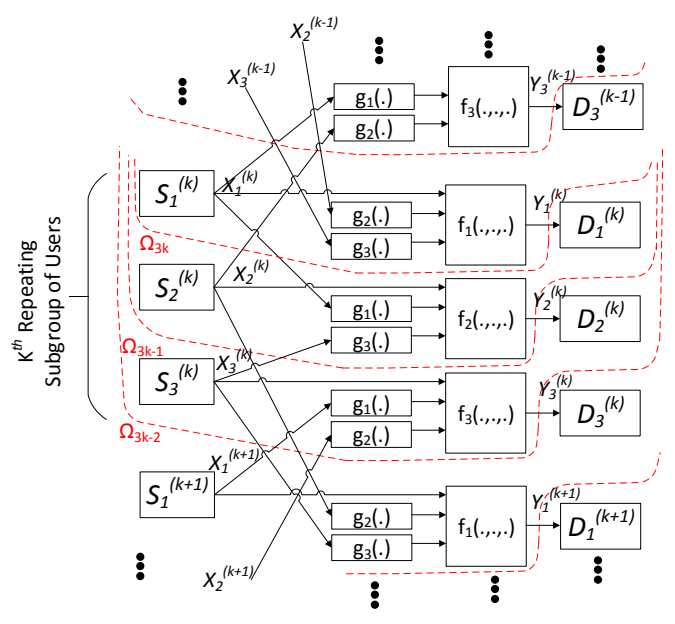

Fig. 13. Extended network and the cuts for deriving bound 23 for the three-user DIC

bound and picking cuts depicted in Fig. 14, we have

$$
\begin{aligned}
& n R_{\Sigma}=n\left(R_{1}+R_{2}+R_{3}+k R_{1}+k R_{2}+k R_{3}\right) \\
& \stackrel{(a)}{\leq} H\left(Y_{\Omega_{1}^{c}}^{n}\right)+\sum_{l=2}^{3 k+2} H\left(Y_{\Omega_{l}^{c}}^{n} \cap \Omega_{l-1} \mid W_{\mathcal{S} \backslash \Omega_{l}}, Y_{\Omega_{l-1}^{c}}^{n}\right)+n \epsilon_{n} \\
& \stackrel{(b)}{\leq} \sum_{i=1}^{n} k H\left(Y_{1}[i] \mid V_{1}[i] V_{3}[i]\right)+k H\left(Y_{2}[i] \mid V_{2}[i] V_{1}[i]\right) \\
& \quad+\sum_{i=1}^{n} k H\left(Y_{3}[i] \mid V_{2}[i], V_{3}[i]\right)+C+n \epsilon_{n}
\end{aligned}
$$

where $(a)$ follows from GCS [8] for deterministic networks and step $(b)$ follows from the proof presented in Appendix B.

By letting $k$ goes to infinity, bound (24) will be recovered.

Derivation of bound (25). To derive this bound, we design the extended network considered in Fig. 15. By applying GCS bound and picking cuts depicted in Fig. 15, we have

$$
\begin{gathered}
n R_{\Sigma}=n\left(R_{1}+R_{2}+R_{3}+k R_{1}+k R_{2}+k R_{3}\right) \\
\stackrel{(a)}{\leq} H\left(Y_{\Omega_{1}^{c}}^{n}\right)+\sum_{l=2}^{3 k+2} H\left(Y_{\Omega_{l}^{c}}^{n} \cap \Omega_{l-1} \mid W_{\mathcal{S} \backslash \Omega_{l}}, Y_{\Omega_{l-1}^{c}}^{n}\right)+n \epsilon_{n} \\
\stackrel{(b)}{\leq} \sum_{i=1}^{n} k H\left(Y_{1}[i] \mid V_{1}[i] V_{2}[i] V_{3}[i]\right)+k H\left(Y_{2}[i] \mid V_{1}[i]\right) \\
\quad+\sum_{i=1}^{n} k H\left(Y_{3}[i] \mid V_{2}[i] V_{3}[i]\right)+C+n \epsilon_{n}
\end{gathered}
$$

where $(a)$ follows from GCS [8] for deterministic networks and step $(b)$ follows from the proof presented in Appendix B.

By letting $k$ goes to infinity, bound (25) will be recovered. Derivation of bound (26). To derive this bound, we design the original network considered in Fig. 16 By applying GCS bound and picking cuts $\Omega_{1}, \Omega_{2}$, and $\Omega_{3}$ depicted in Fig. 16 


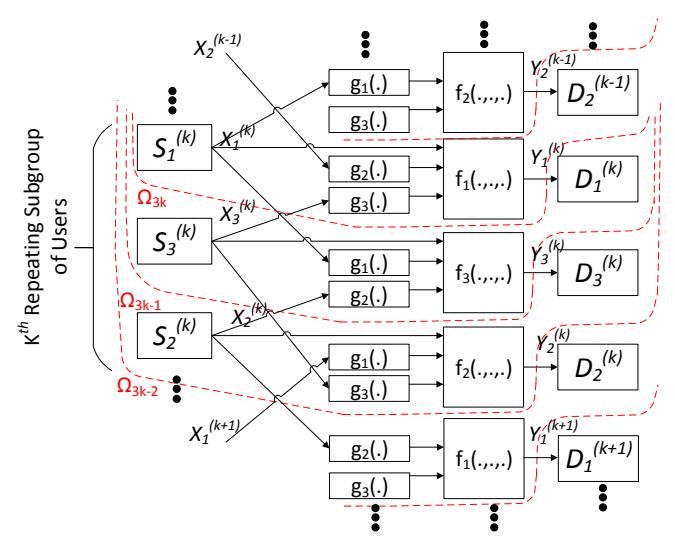

Fig. 14. Extended network and the cuts for deriving bound 24 for the three-user DIC

we have

$$
\begin{aligned}
n R_{\Sigma} & =n\left(R_{1}+R_{2}+R_{3}\right) \\
& \stackrel{(a)}{\leq} H\left(Y_{\Omega_{1}^{c}}^{n}\right)+\sum_{l=2}^{3} H\left(Y_{\Omega_{l}^{c}}^{n} \cap \Omega_{l-1} \mid W_{\mathcal{S} \backslash \Omega_{l}}, Y_{\Omega_{l-1}^{c}}^{n}\right)+n \epsilon_{n} \\
& \stackrel{(c)}{\leq} \sum_{i=1}^{n} H\left(Y_{1}[i] \mid V_{1}[i] V_{2}[i] V_{3}[i]\right)+\sum_{i=1}^{n} H\left(Y_{2}[i] \mid V_{1}[i] V_{2}[i] V_{3}[i]\right) \\
& +\sum_{i=1}^{n} H\left(Y_{3}[i]\right)+n \epsilon_{n}
\end{aligned}
$$

where $(a)$ follows from GCS [8] for deterministic networks and step $(b)$ follows from the proof presented in Appendix B.

Derivation of bound (27). To derive this bound, we design the extended network considered in Fig. 17. Consider $\left\{\mathcal{C}_{n}\right\}$ as a sequence of coding scheme with block length $n$ that achieves sum rate $R_{\Sigma}$ on this extended network. Therefore, by applying GCS bound on this extended network and picking cuts $\Omega_{1}, \Omega_{2}$, $\Omega_{3}$, and $\Omega_{4}$ depicted in Fig. 17, we have

$$
\begin{aligned}
n R_{\Sigma} & =n\left(R_{1}+R_{2}+R_{3}+R_{1}\right) \\
& \stackrel{(a)}{\leq} H\left(Y_{\Omega_{1}^{c}}^{n}\right)+\sum_{l=2}^{4} H\left(Y_{\Omega_{l}^{c}}^{n} \cap \Omega_{l-1} \mid W_{\mathcal{S} \backslash \Omega_{l}}, Y_{\Omega_{l-1}^{c}}^{n}\right)+n \epsilon_{n} \\
& \stackrel{(b)}{\leq} \sum_{i=1}^{n} H\left(Y_{1}[i]\right)+\sum_{i=1}^{n} H\left(Y_{1}[i] \mid V_{1}[i] V_{2}[i] V_{3}[i]\right) \\
& +\sum_{i=1}^{n} H\left(Y_{2}[i] \mid V_{2}[i] V_{3}[i]\right) \\
& +\sum_{i=1}^{n} H\left(Y_{3}[i] \mid V_{1}[i] V_{2}[i] V_{3}[i]\right)+n \epsilon_{n}
\end{aligned}
$$

where (a) follows from GCS [8] for deterministic networks and step $(b)$ follows from the proof presented in Appendix B. Derivation of bound (28). To derive this bound, we design the extended network considered in Fig. 18. By applying GCS

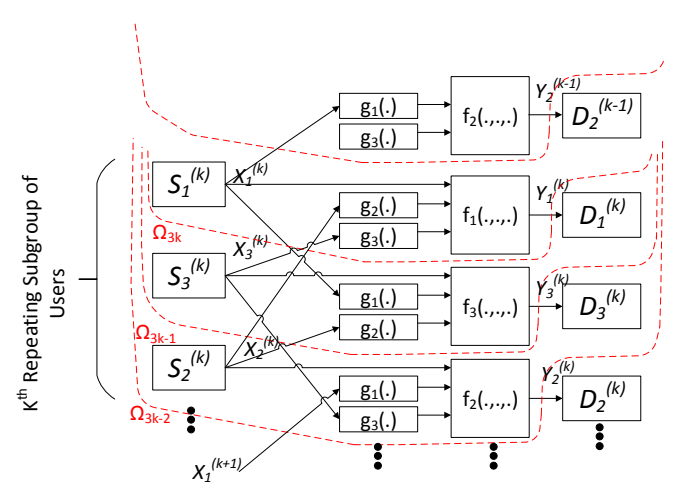

Fig. 15. Extended network and the cuts for deriving bound 25 for the three-user DIC

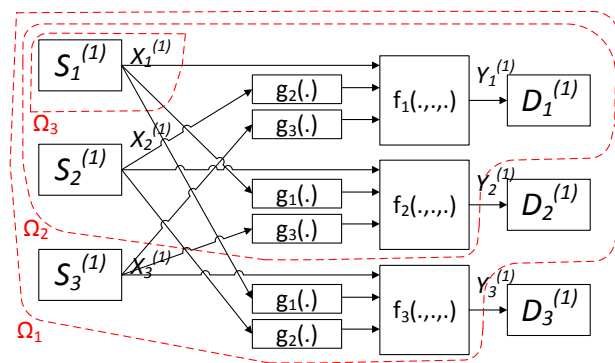

Fig. 16. Original network and the cuts for deriving bound 26 for the three-user DIC

bound and picking cuts depicted in Fig. 18 , we have

$$
\begin{aligned}
n R_{\Sigma} & =n\left(R_{1}+R_{2}+R_{3}+2 k R_{1}+k R_{2}+k R_{3}\right) \\
& \stackrel{(a)}{\leq} H\left(Y_{\Omega_{1}^{c}}^{n}\right)+\sum_{l=2}^{4 k+2} H\left(Y_{\Omega_{l}^{c}}^{n} \cap \Omega_{l-1} \mid W_{\mathcal{S} \backslash \Omega_{l}}, Y_{\Omega_{l-1}^{c}}^{n}\right)+n \epsilon_{n} \\
& \stackrel{(b)}{\leq} \sum_{i=1}^{n} k H\left(Y_{1}[i] \mid V_{1}[i]\right)+k H\left(Y_{1}[i] \mid V_{1}[i] V_{2}[i] V_{3}[i]\right) \\
& +\sum_{i=1}^{n} k H\left(Y_{2}[i] \mid V_{2}[i] V_{3}[i]\right)+k H\left(Y_{3}[i] \mid V_{2}[i] V_{3}[i]\right) \\
& +C+n \epsilon_{n}
\end{aligned}
$$




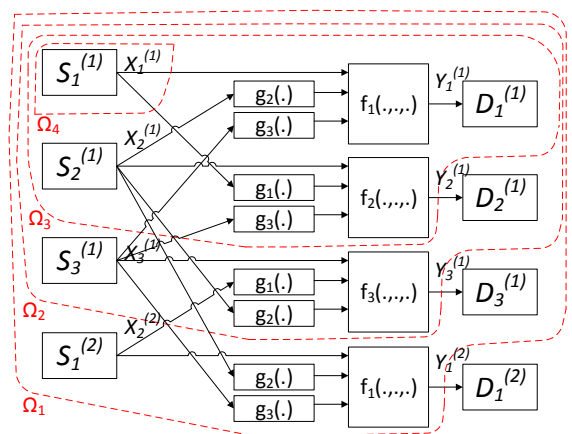

Fig. 17. Extended network and the cuts for deriving bound 27 for the three-user DIC

where $(a)$ follows from GCS [8] for deterministic networks and step $(b)$ follows from the proof presented in Appendix B.

By letting $k$ goes to infinity, bound (28) will be recovered.

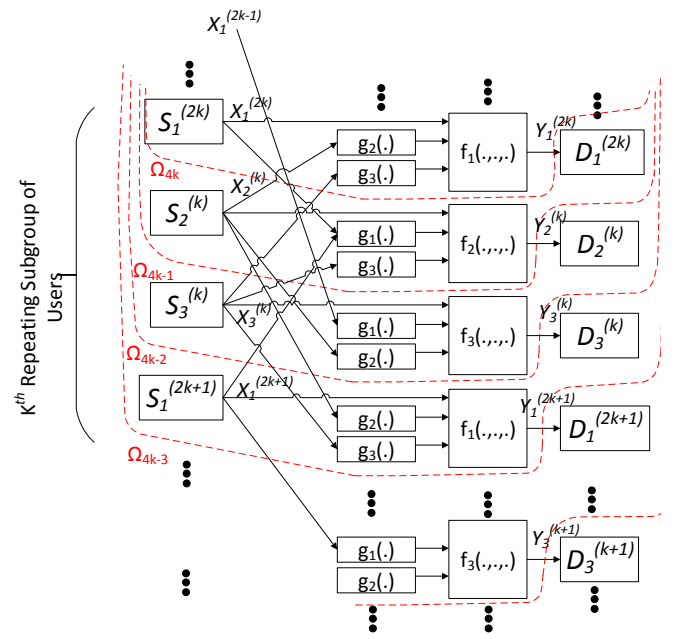

Fig. 18. Extended network and the cuts for deriving bound 28 for the three-user DIC

Derivation of bound (29). To derive this bound, we design the extended network considered in Fig. 19. By applying GCS bound and picking cuts depicted in Fig. 19, we have

$$
\begin{aligned}
n R_{\Sigma} & =n\left(R_{1}+R_{2}+R_{3}+2 k R_{1}+k R_{2}+k R_{3}\right) \\
& \stackrel{(a)}{\leq} H\left(Y_{\Omega_{1}^{c}}^{n}\right)+\sum_{l=2}^{4 k+2} H\left(Y_{\Omega_{l}^{c} \cap \Omega_{l-1}}^{n} \mid W_{\mathcal{S} \backslash \Omega_{l}}, Y_{\Omega_{l-1}^{c}}^{n}\right)+n \epsilon_{n} \\
& \stackrel{(b)}{\leq} \sum_{i=1}^{n} k H\left(Y_{1}[i] \mid V_{1}[i] V_{3}[i]\right)+k H\left(Y_{1}[i] \mid V_{2}[i]\right) \\
& +\sum_{i=1}^{n} k H\left(Y_{2}[i] \mid V_{1}[i] V_{2}[i] V_{3}[i]\right)+k H\left(Y_{3}[i] \mid V_{2}[i] V_{3}[i]\right) \\
& +C+n \epsilon_{n}
\end{aligned}
$$

where $(a)$ follows from GCS [8] for deterministic networks and step $(b)$ follows from the proof presented in Appendix B.

By letting $k$ goes to infinity, bound 29, will be recovered.

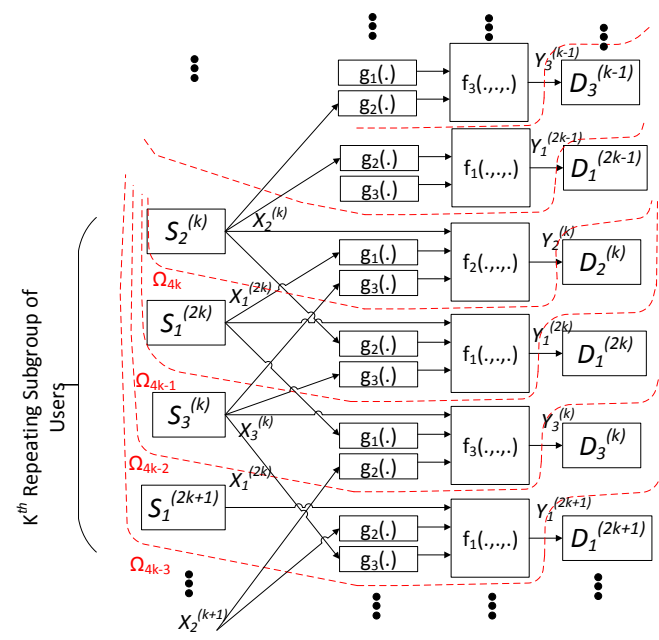

Fig. 19. Extended network and the cuts for deriving bound 29] for the three-user DIC

Derivation of bound (30). To derive this bound, we design the extended network considered in Fig. 20. By applying GCS bound and picking cuts depicted in Fig. 20, we have

$$
\begin{aligned}
n R_{\Sigma} & =n\left(R_{1}+R_{2}+R_{3}+2 k R_{1}+k R_{2}+k R_{3}\right) \\
& \stackrel{(a)}{\leq} H\left(Y_{\Omega_{1}^{c}}^{n}\right)+\sum_{l=2}^{4 k+2} H\left(Y_{\Omega_{l}^{c}}^{n} \cap \Omega_{l-1} \mid W_{\mathcal{S} \backslash \Omega_{l}}, Y_{\Omega_{l-1}^{c}}^{n}\right)+n \epsilon_{n} \\
& \stackrel{(c)}{\leq} \sum_{i=1}^{n} k H\left(Y_{1}[i] \mid V_{1}[i] V_{2}[i] V_{3}[i]\right)+k H\left(Y_{1}[i] \mid V_{3}[i]\right) \\
& +\sum_{i=1}^{n} k H\left(Y_{2}[i] \mid V_{1}[i] V_{2}[i]\right)+k H\left(Y_{3}[i] \mid V_{2}[i] V_{3}[i]\right) \\
& +C+n \epsilon_{n}
\end{aligned}
$$

where $(a)$ follows from GCS [8] for deterministic networks and step $(b)$ follows from the proof presented in Appendix B.

By letting $k$ goes to infinity, bound (30) will be recovered. Derivation of bound (31). To derive this bound, we design the extended network considered in Fig. 21. By applying GCS bound and picking cuts depicted in Fig. 21] we have

$$
\begin{aligned}
n R_{\Sigma} & =n\left(R_{1}+R_{2}+R_{3}+2 k R_{1}+k R_{2}+k R_{3}\right) \\
& \stackrel{(a)}{\leq} H\left(Y_{\Omega_{1}^{c}}^{n}\right)+\sum_{l=2}^{4 k+2} H\left(Y_{\Omega_{l}^{c}}^{n} \cap \Omega_{l-1} \mid W_{\mathcal{S} \backslash \Omega_{l}}, Y_{\Omega_{l-1}^{c}}^{n}\right)+n \epsilon_{n} \\
& \stackrel{(b)}{\leq} \sum_{i=1}^{n} k H\left(Y_{1}[i] \mid V_{1}[i] V_{2}[i] V_{3}[i]\right)+k H\left(Y_{1}[i] \mid V_{3}[i]\right) \\
& +\sum_{i=1}^{n} k H\left(Y_{2}[i] \mid V_{2}[i]\right)+k H\left(Y_{3}[i] \mid V_{1}[i] V_{2}[i] V_{3}[i]\right) \\
& +C+n \epsilon_{n}
\end{aligned}
$$




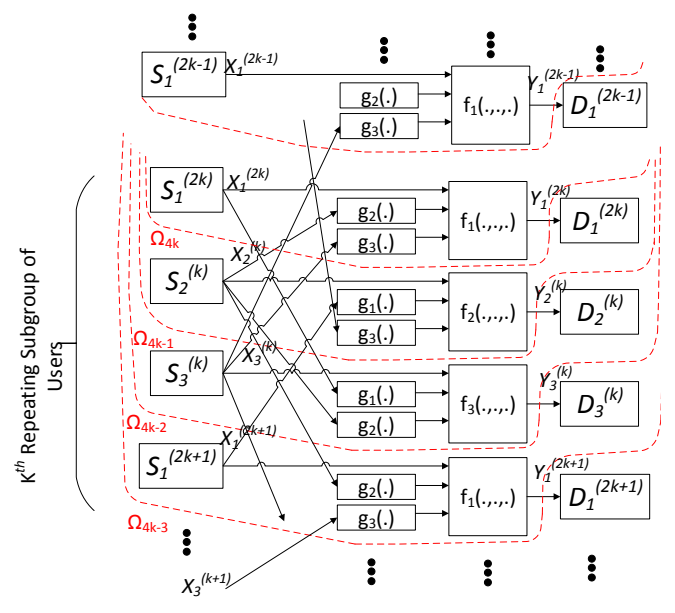

Fig. 20. Extended network and the cuts for deriving bound 30 for the three-user DIC

where $(a)$ follows from GCS [8] for deterministic networks and step (b) follows from the proof presented in Appendix B.

By letting $k$ goes to infinity, bound (31) will be recovered.

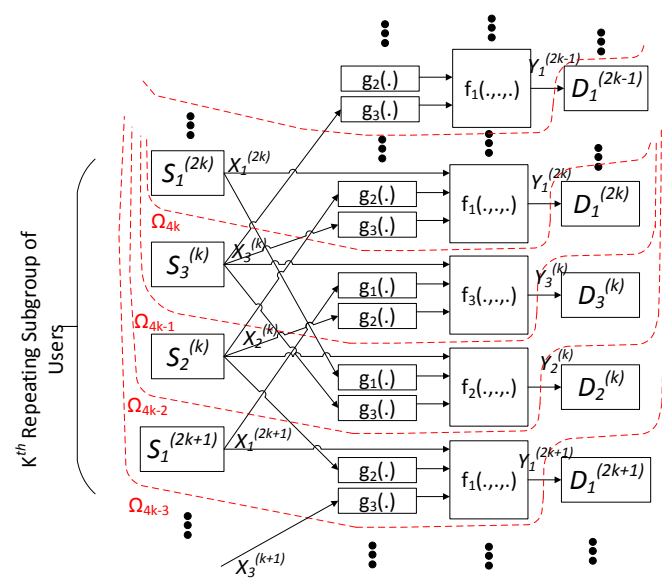

Fig. 21. Extended network and the cuts for deriving bound 31 for the three-user DIC

Derivation of bound (32). To derive this bound, we design the extended network considered in Fig. 22. By applying GCS bound and picking cuts depicted in Fig. 22, we have

$$
\begin{aligned}
n R_{\Sigma} & =n\left(R_{1}+R_{2}+R_{3}+2 k R_{1}+k R_{2}+k R_{3}\right) \\
& \stackrel{(a)}{\leq} H\left(Y_{\Omega_{1}^{c}}^{n}\right)+\sum_{l=2}^{4 k+2} H\left(Y_{\Omega_{l}^{c}}^{n} \cap \Omega_{l-1} \mid W_{\mathcal{S} \backslash \Omega_{l}}, Y_{\Omega_{l-1}^{c}}^{n}\right)+n \epsilon_{n} \\
& \stackrel{(b)}{\leq} \sum_{i=1}^{n} k H\left(Y_{1}[i] \mid V_{1}[i] V_{2}[i] V_{3}[i]\right)+k H\left(Y_{1}[i] \mid V_{1}[i] V_{2}[i]\right) \\
& +\sum_{i=1}^{n} k H\left(Y_{2}[i] \mid V_{2}[i] V_{3}[i]\right)+k H\left(Y_{3}[i] \mid V_{3}[i]\right) \\
& +C+n \epsilon_{n}
\end{aligned}
$$

where $(a)$ follows from GCS [8] for deterministic networks and step $(b)$ follows from the proof presented in Appendix B.

By letting $k$ goes to infinity, bound (32) will be recovered.

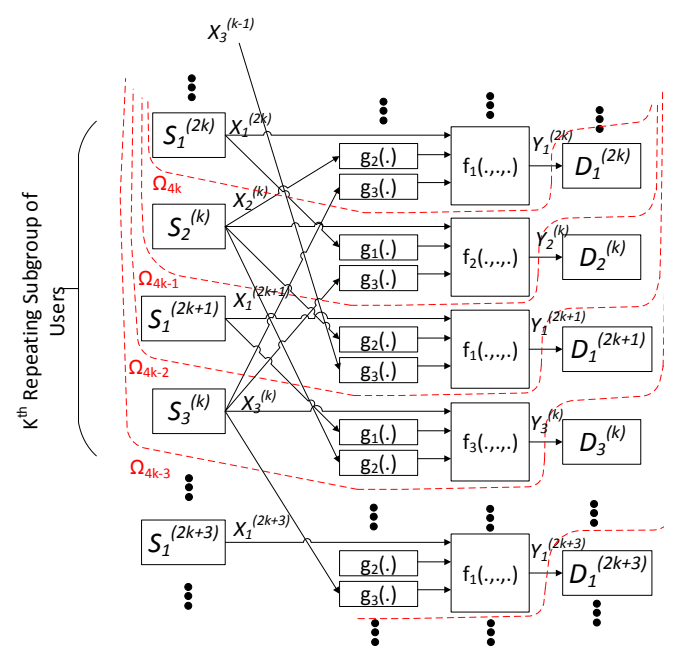

Fig. 22. Extended network and the cuts for deriving bound 32 for the three-user DIC

Derivation of bound (33). To derive this bound, we design the extended network considered in Fig. 23. By applying GCS bound and picking cuts $\Omega_{1}, \Omega_{2}, \Omega_{3}$, and $\Omega_{4}$ depicted in Fig. 23. we have

$$
\begin{aligned}
n R_{\Sigma} & =n\left(R_{1}+R_{2}+R_{3}+R_{1}\right) \\
& \stackrel{(a)}{\leq} H\left(Y_{\Omega_{1}^{c}}^{n}\right)+\sum_{l=2}^{4} H\left(Y_{\Omega_{l}^{c}}^{n} \cap \Omega_{l-1} \mid W_{\mathcal{S} \backslash \Omega_{l}}, Y_{\Omega_{l-1}^{c}}^{n}\right)+n \epsilon_{n} \\
& \stackrel{(b)}{\leq} \sum_{i=1}^{n} 2 H\left(Y_{1}[i] \mid V_{1}[i] V_{2}[i] V_{3}[i]\right)+\sum_{i=1}^{n} H\left(Y_{2}[i]\right) \\
& +\sum_{i=1}^{n} H\left(Y_{3}[i] \mid V_{2}[i] V_{3}[i]\right)+n \epsilon_{n}
\end{aligned}
$$

where $(a)$ follows from GCS [8] for deterministic networks and step $(b)$ follows from the proof presented in Appendix B.

Derivation of bound (34). To derive this bound, we design the extended network considered in Fig. 24. By applying GCS 


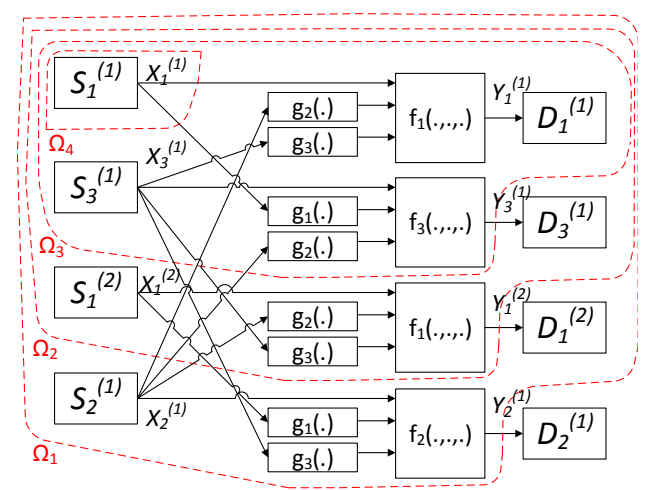

Fig. 23. Extended network and the cuts for deriving bound 33 for the three-user DIC

bound and picking cuts depicted in Fig. 24, we have

$$
\begin{aligned}
n R_{\Sigma} & =n\left(R_{1}+R_{2}+R_{3}+2 k R_{1}+k R_{2}+k R_{3}\right) \\
& \stackrel{(a)}{\leq} H\left(Y_{\Omega_{1}^{c}}^{n}\right)+\sum_{l=2}^{4 k+2} H\left(Y_{\Omega_{l}^{c} \cap \Omega_{l-1}}^{n} \mid W_{\mathcal{S} \backslash \Omega_{l}}, Y_{\Omega_{l-1}^{c}}^{n}\right)+n \epsilon_{n} \\
& \stackrel{(b)}{\leq} \sum_{i=1}^{n} 2 k H\left(Y_{1}[i] \mid V_{1}[i] V_{2}[i] V_{3}[i]\right)+k H\left(Y_{2}[i] \mid V_{2}[i]\right) \\
& +\sum_{i=1}^{n} k H\left(Y_{3}[i] \mid V_{3}[i]\right)+C+n \epsilon_{n}
\end{aligned}
$$

where $(a)$ follows from GCS [8] for deterministic networks and step $(b)$ follows from the proof presented in Appendix B.

By letting $k$ goes to infinity, bound (34) will be recovered.

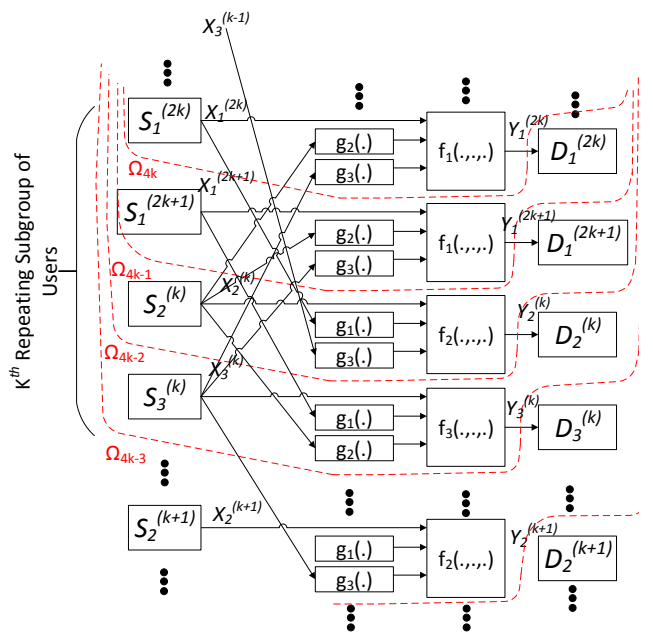

Fig. 24. Extended network and the cuts for deriving bound 34 for the three-user DIC

Derivation of bound (35). To derive this bound, we design the extended network considered in Fig. 25. By applying GCS bound and picking cuts depicted in Fig. 25, we have

$$
\begin{aligned}
n R_{\Sigma} & =n\left(3 R_{1}+R_{2}+R_{3}\right) \\
& \stackrel{(a)}{\leq} H\left(Y_{\Omega_{1}^{c}}^{n}\right)+\sum_{l=2}^{5} H\left(Y_{\Omega_{l}^{c}}^{n} \cap \Omega_{l-1} \mid W_{\mathcal{S} \backslash \Omega_{l}}, Y_{\Omega_{l-1}^{c}}^{n}\right)+n \epsilon_{n} \\
& \stackrel{(b)}{\leq} \sum_{i=1}^{n} 2 H\left(Y_{1}[i] \mid V_{1}[i] V_{2}[i] V_{3}[i]\right)+H\left(Y_{1}[i]\right) \\
& +\sum_{i=1}^{n} H\left(Y_{2}[i] \mid V_{2}[i] V_{3}[i]\right)+H\left(Y_{3}[i] \mid V_{2}[i] V_{3}[i]\right)+n \epsilon_{n}
\end{aligned}
$$

where $(a)$ follows from GCS [8] for deterministic networks and step $(b)$ follows from the proof presented in Appendix B.

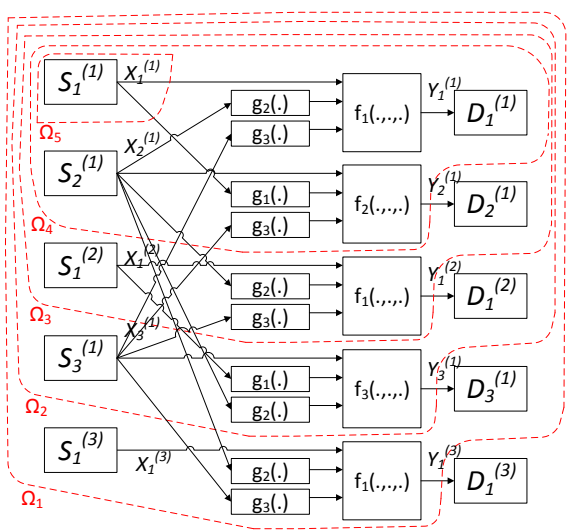

Fig. 25. Extended network and the cuts for deriving bound 35 for the three-user DIC

Derivation of bound (36). To derive this bound, we design the extended network considered in Fig. 26. By applying GCS bound and picking cuts depicted in Fig. 26, we have

$$
\begin{aligned}
n R_{\Sigma} & =n\left(R_{1}+R_{2}+R_{3}+3 k R_{1}+k R_{2}+k R_{3}\right) \\
& \stackrel{(a)}{\leq} H\left(Y_{\Omega_{1}^{c}}^{n}\right)+\sum_{l=2}^{5 k+2} H\left(Y_{\Omega_{l}^{c}}^{n} \cap \Omega_{l-1} \mid W_{\mathcal{S} \backslash \Omega_{l}}, Y_{\Omega_{l-1}^{c}}^{n}\right)+n \epsilon_{n} \\
& \stackrel{(b)}{\leq} \sum_{i=1}^{n} 2 k H\left(Y_{1}[i] \mid V_{1}[i] V_{2}[i] V_{3}[i]\right)+k H\left(Y_{1}[i] \mid V_{2}[i]\right) \\
& +\sum_{i=1}^{n} k H\left(Y_{2}[i] \mid V_{2}[i] V_{3}[i]\right)+k H\left(Y_{3}[i] \mid V_{3}[i]\right) \\
& +C+n \epsilon_{n}
\end{aligned}
$$

where $(a)$ follows from GCS [8] for deterministic networks and step $(b)$ follows from the proof presented in Appendix B.

By letting $k$ goes to infinity, bound (36) will be recovered.

Derivation of bound (37). To derive this bound, we design the extended network considered in Fig. 28 By applying GCS bound and picking cuts $\Omega_{1}, \ldots, \Omega_{5}$ depicted in Fig. 9 , we have 


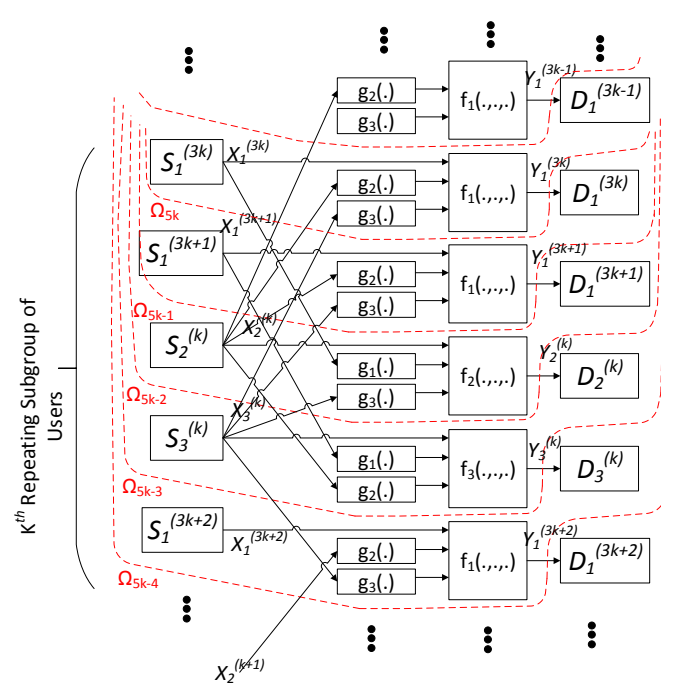

Fig. 26. Extended network and the cuts for deriving bound 36 for the three-user DIC

$$
\begin{aligned}
n R_{\Sigma} & =n\left(2 R_{1}+2 R_{2}+R_{3}\right) \\
& \stackrel{(a)}{\leq} H\left(Y_{\Omega_{1}^{c}}^{n}\right)+\sum_{l=2}^{5} H\left(Y_{\Omega_{l}^{c}}^{n} \cap \Omega_{l-1} \mid W_{\mathcal{S} \backslash \Omega_{l}}, Y_{\Omega_{l-1}^{c}}^{n}\right)+n \epsilon_{n} \\
& \left.\stackrel{(b)}{\leq} \sum_{i=1}^{n} Y_{1^{(1)}}[i]\right)+\sum_{i=1}^{n} H\left(Y_{3^{(1)}}[i] \mid V_{3^{(1)}}[i] V_{2^{(1)}}[i]\right) \\
& +\sum_{i=1}^{n} H\left(Y_{1^{(1)}}[i] \mid V_{1^{(1)}}[i] V_{3^{(1)}}[i]\right) \\
& +2 \sum_{i=1}^{n} H\left(Y_{2^{(1)}}[i] \mid V_{2^{(1)}}[i] V_{1^{(1)}}[i] V_{3^{(1)}}[i]\right)+n \epsilon_{n}
\end{aligned}
$$

where $(a)$ follows from GCS [8] for deterministic networks and step $(b)$ follows from the proof presented in Appendix B.

Derivation of bound (38). To derive this bound, we design the extended network considered in Fig. 28. By applying GCS bound and picking cuts $\Omega_{1}, \ldots, \Omega_{5}$ depicted in Fig. 28, we have

$$
\begin{aligned}
n R_{\Sigma} & =n\left(2 R_{1}+2 R_{2}+R_{3}\right) \\
& \stackrel{(a)}{\leq} H\left(Y_{\Omega_{1}^{c}}^{n}\right)+\sum_{l=2}^{5} H\left(Y_{\Omega_{l}^{c}}^{n} \cap \Omega_{l-1} \mid W_{\mathcal{S} \backslash \Omega_{l}}, Y_{\Omega_{l-1}^{c}}^{n}\right)+n \epsilon_{n} \\
& \stackrel{(b)}{\leq} \sum_{i=1}^{n} H\left(Y_{1}[i]\right)+H\left(Y_{1}[i] \mid V_{1}[i] V_{2}[i] V_{3}[i]\right) \\
& +\sum_{i=1}^{n} H\left(Y_{2}[i] \mid V_{1}[i] V_{2}[i] V_{3}[i]\right)+H\left(Y_{2}[i] \mid V_{2}[i] V_{3}[i]\right) \\
& +\sum_{i=1}^{n} H\left(Y_{3}[i] \mid V_{1}[i] V_{3}[i]\right)+n \epsilon_{n}
\end{aligned}
$$

where (a) follows from GCS [8] for deterministic networks

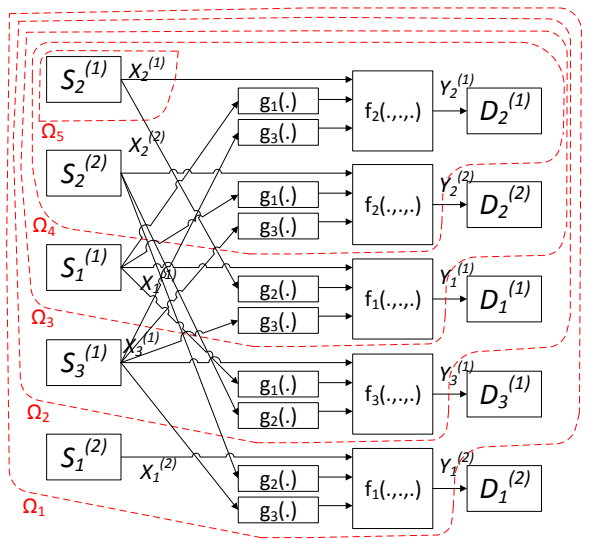

Fig. 27. Extended network and the cuts for deriving bound 37 for the three-user DIC

and step $(b)$ follows from the proof presented in Appendix B.

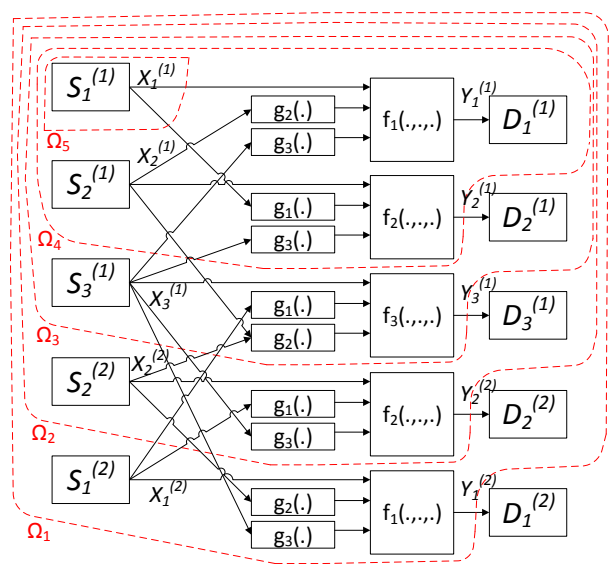

Fig. 28. Extended network and the cuts for deriving bound 38 for the three-user DIC

Derivation of bound (39). To derive this bound, we design the extended network considered in Fig. 29. By applying GCS bound and picking cuts $\Omega_{1}, \ldots, \Omega_{5}$ depicted in Fig. 29, we have

$$
\begin{aligned}
n R_{\Sigma} & =n\left(2 R_{1}+2 R_{2}+R_{3}\right) \\
& \stackrel{(a)}{\leq} H\left(Y_{\Omega_{1}^{c}}^{n}\right)+\sum_{l=2}^{5} H\left(Y_{\Omega_{l}^{c} \cap \Omega_{l-1}}^{n} \mid W_{\mathcal{S} \backslash \Omega_{l}}, Y_{\Omega_{l-1}^{c}}^{n}\right)+n \epsilon_{n} \\
& \stackrel{(b)}{\leq} \sum_{i=1}^{n} H\left(Y_{1}[i]\right)+H\left(Y_{1}[i] \mid V_{1}[i] V_{2}[i] V_{3}[i]\right) \\
& +\sum_{i=1}^{n} 2 H\left(Y_{2}[i] \mid V_{1}[i] V_{2}[i] V_{3}[i]\right)+H\left(Y_{3}[i] \mid V_{3}[i]\right)+n \epsilon_{n}
\end{aligned}
$$

where $(a)$ follows from GCS [8] for deterministic networks and step $(b)$ follows from the proof presented in Appendix B.

Derivation of bound (40). To derive this bound, we design the extended network considered in Fig. 30. By applying GCS 


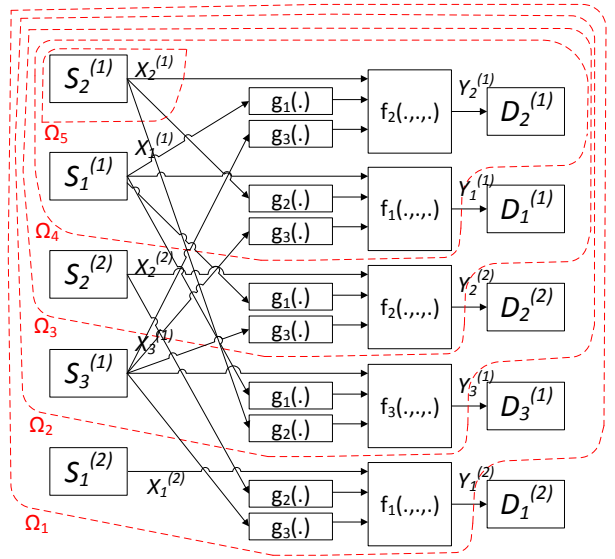

Fig. 29. Extended network and the cuts for deriving bound 39 for the three-user DIC

bound and picking cuts depicted in Fig. 30, we have

$$
\begin{aligned}
n R_{\Sigma} & =n\left(R_{1}+R_{2}+R_{3}+2 k R_{1}+2 k R_{2}+k R_{3}\right) \\
& \stackrel{(a)}{\leq} H\left(Y_{\Omega_{1}^{c}}^{n}\right)+\sum_{l=2}^{5 k+2} H\left(Y_{\Omega_{l}^{c} \cap \Omega_{l-1}}^{n} \mid W_{\mathcal{S} \backslash \Omega_{l}}, Y_{\Omega_{l-1}^{c}}^{n}\right)+n \epsilon_{n} \\
& \stackrel{(b)}{\leq} \sum_{i=1}^{n} k H\left(Y_{1}[i] \mid V_{1}[i]\right)+k H\left(Y_{1}[i] \mid V_{1}[i] V_{2}[i] V_{3}[i]\right) \\
& +\sum_{i=1}^{n} k H\left(Y_{2}[i] \mid V_{1}[i] V_{2}[i] V_{3}[i]\right) \\
& +\sum_{i=1}^{n} k H\left(Y_{2}[i] \mid V_{2}[i] V_{3}[i]\right)+k H\left(Y_{3}[i] \mid V_{3}[i]\right) \\
& +C+n \epsilon_{n}
\end{aligned}
$$

where $(a)$ follows from GCS [8] for deterministic networks and step $(b)$ follows from the proof presented in Appendix B.

By letting $k$ goes to infinity, bound (40) will be recovered. Derivation of bound (41). To derive this bound, we design the extended network considered in Fig. 31. By applying GCS bound and picking cuts depicted in Fig. 31, we have

$$
\begin{aligned}
n R_{\Sigma} & =n\left(R_{1}+R_{2}+R_{3}+2 k R_{1}+2 k R_{2}+k R_{3}\right) \\
& \stackrel{(a)}{\leq} H\left(Y_{\Omega_{1}^{c}}^{n}\right)+\sum_{l=2}^{5 k+2} H\left(Y_{\Omega_{l}^{c}}^{n} \cap \Omega_{l-1} \mid W_{\mathcal{S} \backslash \Omega_{l}}, Y_{\Omega_{l-1}^{c}}^{n}\right)+n \epsilon_{n} \\
& \stackrel{(b)}{\leq} \sum_{i=1}^{n} 2 k H\left(Y_{1}[i] \mid V_{1}[i] V_{3}[i]\right)+k H\left(Y_{2}[i] \mid V_{1}[i] V_{2}[i] V_{3}[i]\right) \\
& +\sum_{i=1}^{n} 2 k H\left(Y_{2}[i] \mid V_{2}[i]\right)+k H\left(Y_{3}[i] \mid V_{2}[i] V_{3}[i]\right) \\
& +C+n \epsilon_{n}
\end{aligned}
$$

where $(a)$ follows from GCS [8] for deterministic networks and step $(b)$ follows from the proof presented in Appendix B.

By letting $k$ goes to infinity, bound (41) will be recovered.

Derivation of bound (42). To derive this bound, we design the extended network considered in Fig. 32. By applying GCS

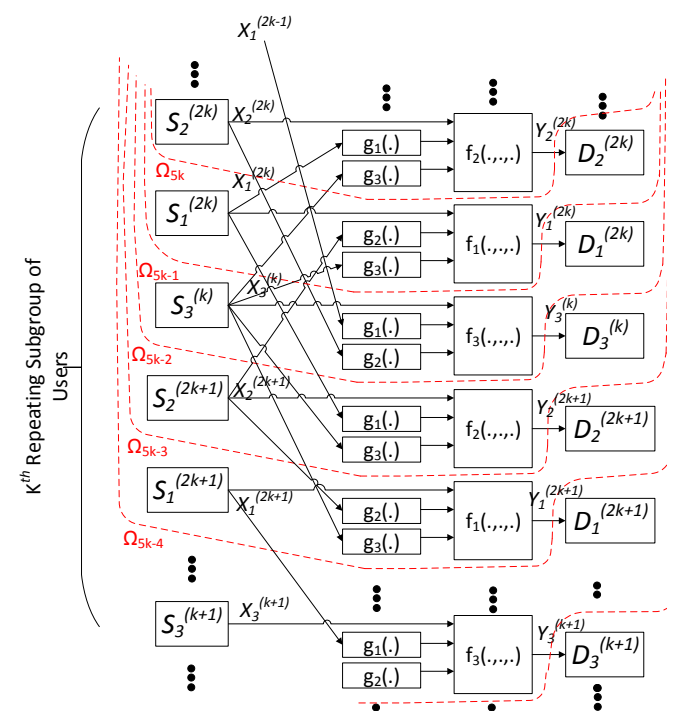

Fig. 30. Extended network and the cuts for deriving bound 40 for the three-user DIC

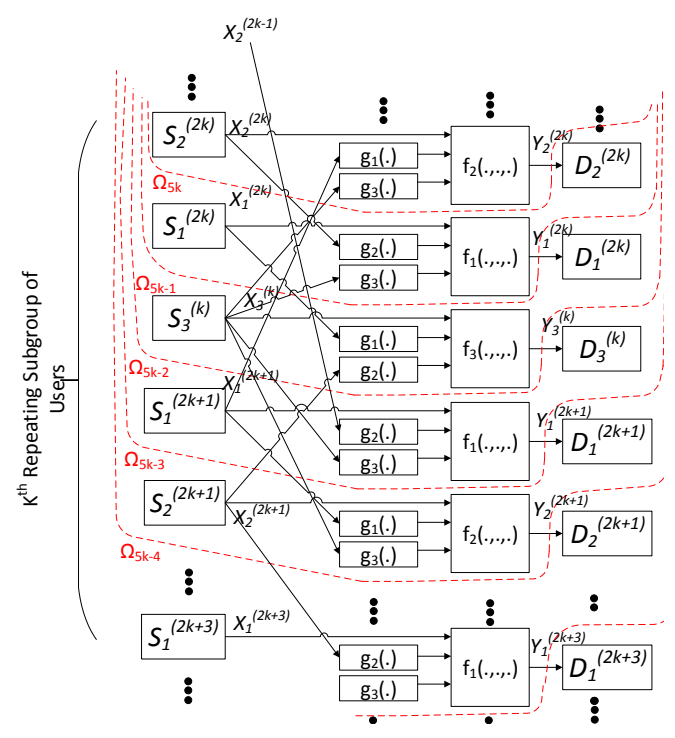

Fig. 31. Extended network and the cuts for deriving bound 41 for the three-user DIC

bound and picking cuts $\Omega_{1}, \ldots, \Omega_{6}$ depicted in Fig. 32, we have $n R_{\Sigma}=n\left(3 R_{1}+2 R_{2}+R_{3}\right)$

$$
\begin{aligned}
& \stackrel{(a)}{\leq} H\left(Y_{\Omega_{1}^{c}}^{n}\right)+\sum_{l=2}^{6} H\left(Y_{\Omega_{l}^{c}}^{n} \cap \Omega_{l-1} \mid W_{\mathcal{S} \backslash \Omega_{l}}, Y_{\Omega_{l-1}^{c}}^{n}\right)+n \epsilon_{n} \\
& \stackrel{(b)}{\leq} \sum_{i=1}^{n} 2 H\left(Y_{1}[i] \mid V_{1}[i] V_{2}[i] V_{3}[i]\right)+H\left(Y_{1}[i]\right) \\
& +\sum_{i=1}^{n} H\left(Y_{2}[i] \mid V_{1}[i] V_{2}[i] V_{3}[i]\right)+H\left(Y_{2}[i] \mid V_{2}[i] V_{3}[i]\right) \\
& +\sum_{i=1}^{n} H\left(Y_{3}[i] \mid V_{3}[i]\right)+n \epsilon_{n}
\end{aligned}
$$


where $(a)$ follows from GCS [8] for deterministic networks and step $(b)$ follows from the proof presented in Appendix B.

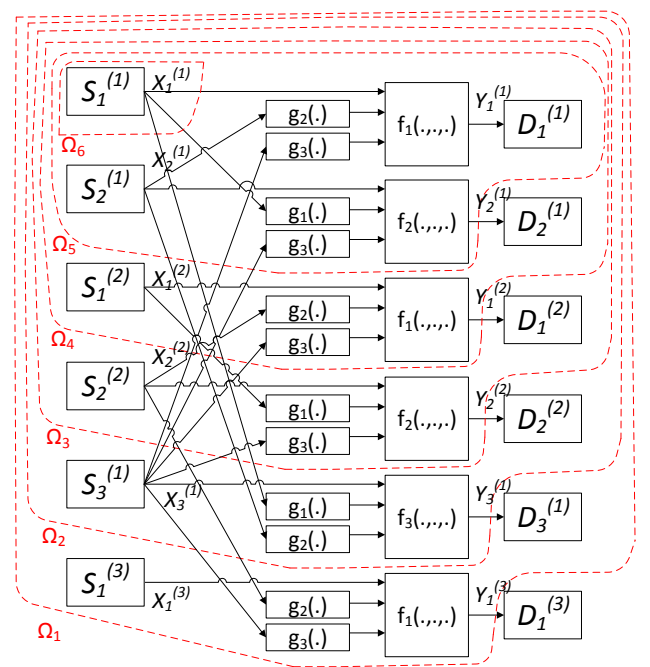

Fig. 32. Extended network and the cuts for deriving bound 42 for the three-user DIC

Derivation of bound (43). To derive this bound, we design the extended network considered in Fig. 33 By applying GCS bound and picking cuts $\Omega_{1}, \ldots, \Omega_{6}$ depicted in Fig. 33 , we have

$$
\begin{aligned}
n R_{\Sigma} & =n\left(3 R_{1}+2 R_{2}+R_{3}\right) \\
& \stackrel{(a)}{\leq} H\left(Y_{\Omega_{1}^{c}}^{n}\right)+\sum_{l=2}^{6} H\left(Y_{\Omega_{l}^{c}}^{n} \cap \Omega_{l-1} \mid W_{\mathcal{S} \backslash \Omega_{l}}, Y_{\Omega_{l-1}^{c}}^{n}\right)+n \epsilon_{n} \\
& \stackrel{(b)}{\leq} \sum_{i=1}^{n} 2 H\left(Y_{1}[i] \mid V_{1}[i] V_{2}[i] V_{3}[i]\right)+H\left(Y_{1}[i]\right) \\
& +\sum_{i=1}^{n} 2 H\left(Y_{2}[i] \mid V_{2}[i] V_{3}[i]\right)+H\left(Y_{3}[i] \mid V_{1}[i] V_{3}[i]\right)+n \epsilon_{n}
\end{aligned}
$$

where $(a)$ follows from GCS [8] for deterministic networks and step $(b)$ follows from the proof presented in Appendix B.

Derivation of bound (44). To derive this bound, we design the extended network considered in Fig. 34 By applying GCS bound and picking cuts depicted in Fig. 34, we have

$$
\begin{aligned}
n R_{\Sigma} & =n\left(R_{1}+R_{2}+R_{3}+3 k R_{1}+2 k R_{2}+k R_{3}\right) \\
& \stackrel{(a)}{\leq} H\left(Y_{\Omega_{1}^{c}}^{n}\right)+\sum_{l=2}^{6 k+2} H\left(Y_{\Omega_{l}^{c}}^{n} \cap \Omega_{l-1} \mid W_{\mathcal{S} \backslash \Omega_{l}}, Y_{\Omega_{l-1}^{c}}^{n}\right)+n \epsilon_{n} \\
& \stackrel{(b)}{\leq} \sum_{i=1}^{n} 2 k H\left(Y_{1}[i] \mid V_{1}[i] V_{2}[i] V_{3}[i]\right)+k H\left(Y_{1}[i] \mid V_{1}[i]\right) \\
& +\sum_{i=1}^{n} 2 k H\left(Y_{2}[i] \mid V_{2}[i] V_{3}[i]\right)+k H\left(Y_{3}[i] \mid V_{3}[i]\right) \\
& +C+n \epsilon_{n}
\end{aligned}
$$

where $(a)$ follows from GCS [8] for deterministic networks and step $(b)$ follows from the proof presented in Appendix B.

By letting $k$ goes to infinity, bound (44) will be recovered. Derivation of bound (45). To derive this bound, we design the extended network considered in Fig. 35. By applying GCS

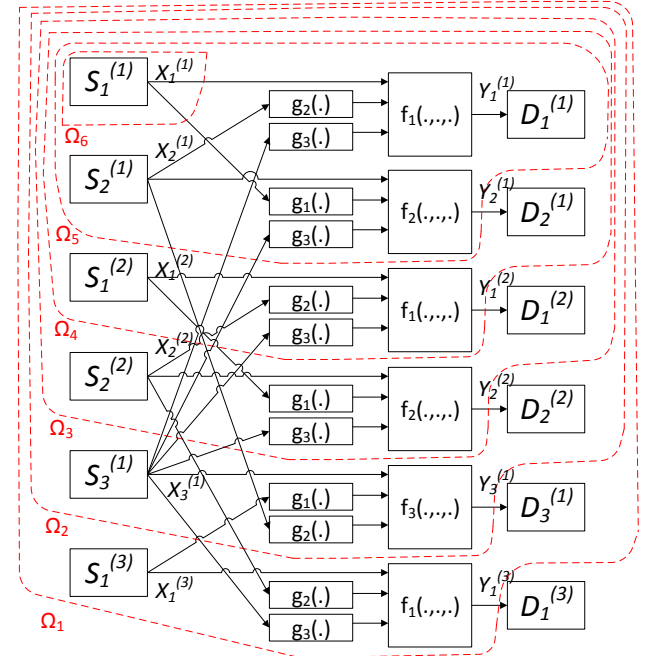

Fig. 33. Extended network and the cuts for deriving bound 43 for the three-user DIC

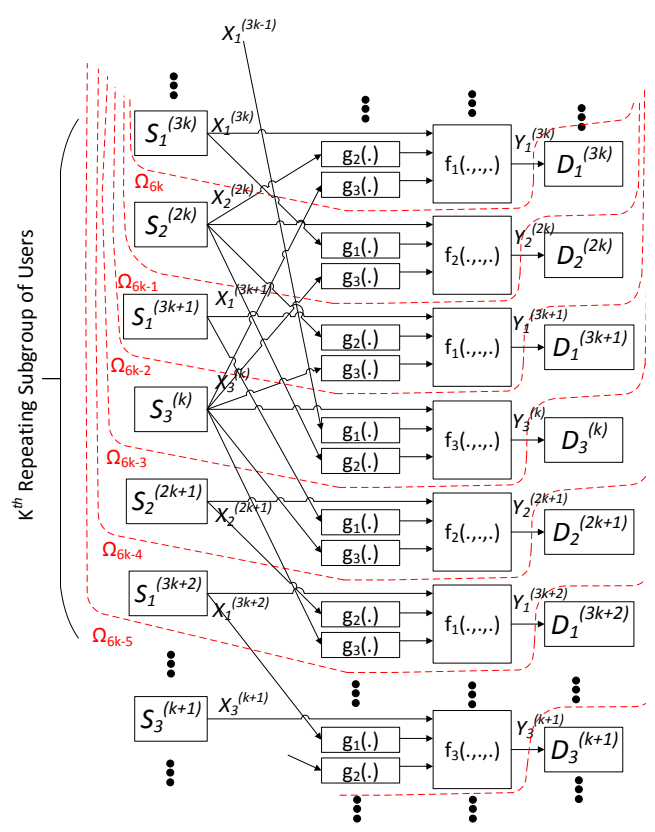

Fig. 34. Extended network and the cuts for deriving bound 44 for the three-user DIC

bound and picking cuts $\Omega_{1}, \ldots, \Omega_{6}$ depicted in Fig. 35, we have $n R_{\Sigma}=n\left(3 R_{1}+2 R_{2}+R_{3}\right)$

$$
\begin{aligned}
& \stackrel{(a)}{\leq} H\left(Y_{\Omega_{1}^{c}}^{n}\right)+\sum_{l=2}^{6} H\left(Y_{\Omega_{l}^{c}}^{n} \cap \Omega_{l-1} \mid W_{\mathcal{S} \backslash \Omega_{l}}, Y_{\Omega_{l-1}^{c}}^{n}\right)+n \epsilon_{n} \\
& \stackrel{(b)}{\leq} \sum_{i=1}^{n} 3 H\left(Y_{1}[i] \mid V_{1}[i] V_{2}[i] V_{3}[i]\right)+H\left(Y_{2}[i] \mid V_{2}[i] V_{3}[i]\right) \\
& +\sum_{i=1}^{n} H\left(Y_{2}[i]\right)+H\left(Y_{3}[i] \mid V_{3}[i]\right)+n \epsilon_{n}
\end{aligned}
$$


where $(a)$ follows from GCS [8] for deterministic networks and step $(b)$ follows from the proof presented in Appendix B.

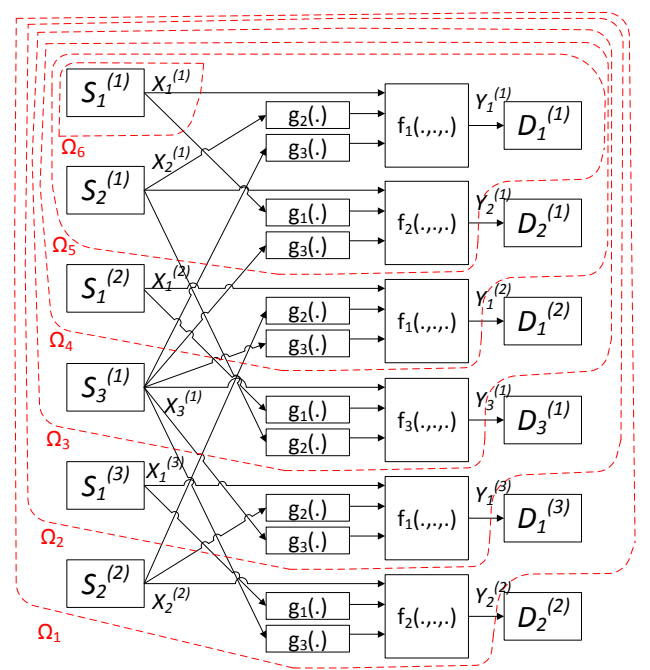

Fig. 35. Extended network and the cuts for deriving bound 45 for the three-user DIC

Derivation of bound (46). To derive this bound, we design the extended network considered in Fig. 36. By applying GCS bound and picking cuts $\Omega_{1}, \ldots, \Omega_{7}$ depicted in Fig. 36, we have

$$
\begin{aligned}
n R_{\Sigma} & =n\left(4 R_{1}+2 R_{2}+R_{3}\right) \\
& \stackrel{(a)}{\leq} H\left(Y_{\Omega_{1}^{c}}^{n}\right)+\sum_{l=2}^{7} H\left(Y_{\Omega_{l}^{c}}^{n} \cap \Omega_{l-1} \mid W_{\mathcal{S} \backslash \Omega_{l}}, Y_{\Omega_{l-1}^{c}}^{n}\right)+n \epsilon_{n} \\
& \stackrel{(b)}{\leq} \sum_{i=1}^{n} 3 H\left(Y_{1}[i] \mid V_{1}[i] V_{2}[i] V_{3}[i]\right)+H\left(Y_{1}[i]\right) \\
& +\sum_{i=1}^{n} 2 H\left(Y_{2}[i] \mid V_{2}[i] V_{3}[i]\right)+H\left(Y_{3}[i] \mid V_{3}[i]\right)+n \epsilon_{n}
\end{aligned}
$$

where $(a)$ follows from GCS [8] for deterministic networks and step $(b)$ follows from the proof presented in Appendix B.

\section{CONCLUSION}

We considered the two-user DIC, proposed the idea of extended networks, and demonstrated that by carefully designing extended networks and applying the GCS bound to them, we can derive a tight converse for the two-user DIC. Furthermore, we generalized our techniques to the three-user DIC, and demonstrated that the proposed approach also results in deriving a tight converse for the symmetric three-user DIC. An interesting future direction can be to investigate whether by applying GCS to extended networks one can establish the capacity region of general DIC. We will characterize the capacity region of symmetric $K$-user DIC in the longer version of this paper.

\section{APPENDIX}

Appendix A

Proof of part (c) for bound (11).

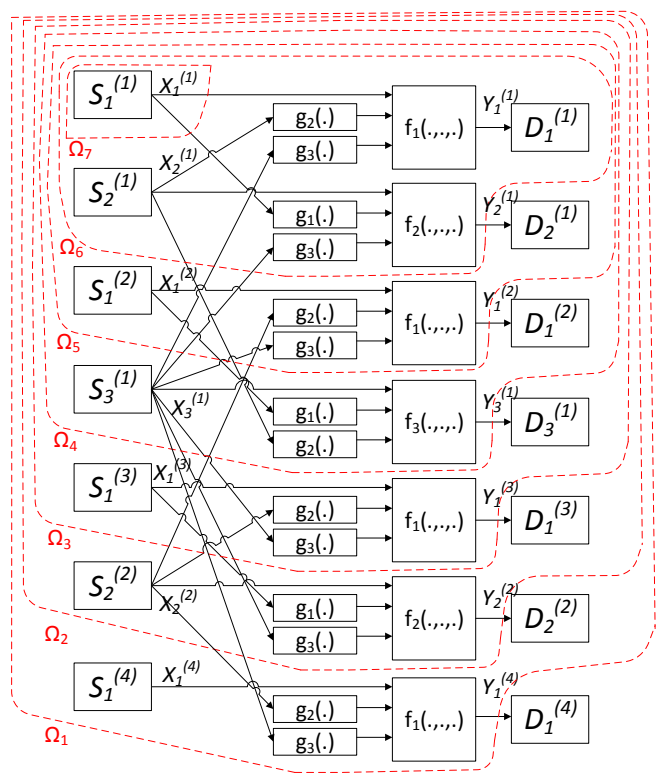

Fig. 36. Extended network and the cuts for deriving bound 46 for the three-user DIC

We first define $X_{i}$ s to be $\left\{X_{i^{(j)}} \mid j \in \mathcal{S}\right\}$ (Similarly for $W_{i^{\mathcal{S}}}$ and $Y_{i}$ s).

$$
\begin{aligned}
& H\left(Y_{\Omega_{1}^{c}}^{n}\right)+\sum_{l=2}^{k+1} H\left(Y_{\Omega_{l}^{c}}^{n} \cap \Omega_{l-1} \mid W_{\mathcal{S} \backslash \Omega_{l}}, Y_{\Omega_{l-1}^{c}}^{n}\right)+n \epsilon_{n} \\
& =H\left(Y_{1(k)}^{n}\right)+\sum_{j=1}^{k-1} H\left(Y_{1(j)}^{n} \mid W_{1[j+1: k]}, Y_{1[j+1: k]}^{n}\right) \\
& +H\left(Y_{2^{(1)}}^{n} \mid W_{1[1: k]}, Y_{1[1: k]}^{n}\right)+n \epsilon_{n} \\
& =H\left(Y_{1(k)}^{n}\right) \\
& +\sum_{j=1}^{k-1} H\left(Y_{1^{(j)}}^{n} \mid W_{1^{[j+1: k]}}, Y_{1^{[j+1: k]}}^{n}, X_{1^{[j+1: k]}}^{n}\right) \\
& +H\left(Y_{2^{(1)}}^{n} \mid W_{1^{[1: k]}}, Y_{1^{[1: k]}}^{n}, X_{1^{[1: k]}}^{n}\right)+n \epsilon_{n} \\
& \leq \sum_{i=1}^{n} H\left(Y_{1^{(k)}}[i] \mid Y_{1^{(k)}}^{i-1}\right) \\
& +\sum_{j=1}^{k-1} \sum_{i=1}^{n} H\left(Y_{1^{(j)}}[i] \mid Y_{1^{(j)}}^{i-1}, Y_{1^{(j+1)}}[i], X_{1^{(j+1)}}[i]\right) \\
& +\sum_{i=1}^{n} H\left(Y_{2^{(1)}}[i] \mid Y_{2^{(1)}}^{i-1}, Y_{1^{(1)}}[i], X_{1^{(1)}}[i]\right)+n \epsilon_{n} \\
& \stackrel{(a)}{\leq} \sum_{i=1}^{n} H\left(Y_{1^{(k)}}[i]\right)+\sum_{j=1}^{k-1} \sum_{i=1}^{n} H\left(Y_{1^{(j)}}[i] \mid V_{2^{(1)}}[i]\right) \\
& +\sum_{i=1}^{n} H\left(Y_{2^{(1)}}[i] \mid V_{2^{(1)}}[i], V_{1^{(1)}}[i]\right)+n \epsilon_{n} \\
& \stackrel{(b)}{\leq} \sum_{i=1}^{n} H\left(Y_{1^{(1)}}[i]\right)+(k-1) \sum_{i=1}^{n} H\left(Y_{1^{(1)}}[i] \mid V_{2^{(1)}}[i]\right) \\
& +\sum_{i=1}^{n} H\left(Y_{2^{(1)}}[i] \mid V_{2^{(1)}}[i], V_{1^{(1)}}[i]\right)+n \epsilon_{n}
\end{aligned}
$$


where step $(a)$ follows from $V_{2^{(1)}}[i]=h_{1}\left(X_{1^{(j)}}[i], Y_{1^{(j)}}[i]\right)$ and $V_{1^{(1)}}[i]=g_{1}\left(X_{1^{(1)}}[i]\right)$. Step $(b)$ follows, since the coding schemes of $S_{1^{(j)}} \mathrm{S}$ are the same, $Y_{1^{(j)}}[i] \mathrm{s}$ have the same probability distribution as $Y_{1^{(1)}}[i]$.

\section{Proof of part (c) for bound (13).}

$$
\begin{aligned}
& H\left(Y_{\Omega_{1}^{c}}^{n}\right)+\sum_{l=2}^{2 k} H\left(Y_{\Omega_{l}^{c}}^{n} \cap \Omega_{l-1} \mid W_{\mathcal{S} \backslash \Omega_{l}}, Y_{\Omega_{l-1}^{c}}^{n}\right) \\
& =H\left(Y_{2^{(k)}}^{n}\right)+H\left(Y_{1^{(k)}}^{n} \mid W_{2^{(k)}}, Y_{2^{(k)}}^{n}\right) \\
& +\sum_{j=1}^{k-1} H\left(Y_{1^{(j)}}^{n} \mid W_{1^{[j+1: k]}}, W_{2^{[j: k]}}, Y_{1^{[j+1: k]}}^{n}, Y_{2^{[j: k]}}^{n}\right) \\
& +\sum_{j=1}^{k-1} H\left(Y_{2^{(j)}}^{n} \mid W_{1^{[j+1: k]}}, W_{2^{[j+1: k]}}, Y_{1^{[j+1: k]}}^{n}, Y_{2^{[j+1: k]}}^{n}\right) \\
& \leq H\left(Y_{2^{(k)}}^{n}\right)+H\left(Y_{1^{(k)}}^{n} \mid X_{2^{(k)}}^{n}, Y_{2^{(k)}}^{n}\right) \\
& +\sum_{j=1}^{k-1} H\left(Y_{1(j)}^{n} \mid X_{1[j+1: k]}^{n}, X_{2[j: k]}^{n}, Y_{1[j+1: k]}^{n}, Y_{2[j: k]}^{n}\right) \\
& +\sum_{j=1}^{k-1} H\left(Y_{2^{(j)}}^{n} \mid X_{1^{[j+1: k]}}^{n}, X_{2^{[j+1: k]}}^{n}, Y_{1[j+1: k]}^{n}, Y_{2^{[j+1: k]}}^{n}\right) \\
& \stackrel{(a)}{=} H\left(Y_{2^{(k)}}^{n}\right)+\sum_{j=1}^{k} H\left(Y_{1^{(j)}}^{n} \mid X_{2^{(j)}}^{n}, Y_{2^{(j)}}^{n}\right) \\
& \sum_{j=1}^{k-1} H\left(Y_{2^{(j)}}^{n} \mid X_{1^{(j+1)}}^{n}, Y_{1^{(j+1)}}^{n}\right) \\
& =\sum_{i=1}^{n} H\left(Y_{2^{(k)}}[i] \mid Y_{2^{(k)}}^{i-1}\right) \\
& +\sum_{i=1}^{n} \sum_{j=1}^{k} H\left(Y_{1^{(j)}}[i] \mid Y_{1^{(j)}}^{(i-1)}, X_{2^{(j)}}^{n}, Y_{2^{(j)}}^{n}\right) \\
& +\sum_{i=1}^{n} \sum_{j=1}^{k} H\left(Y_{2^{(j)}}[i] \mid Y_{2^{(j)}}^{(i-1)}, X_{1^{(j+1)}}^{n}, Y_{1^{(j+1)}}^{n}\right) \\
& \stackrel{(b)}{\leq} \sum_{i=1}^{n} H\left(Y_{2^{(k)}}[i]\right)+\sum_{j=1}^{k} \sum_{i=1}^{n} H\left(Y_{1^{(j)}}[i] \mid V_{1^{(j)}}[i]\right) \\
& +\sum_{j=1}^{k-1} \sum_{i=1}^{n} H\left(Y_{2^{(j)}}[i] \mid V_{2^{(j)}}[i]\right) \\
& \stackrel{(c)}{=} \sum_{i=1}^{n} H\left(Y_{2^{(1)}}[i]\right)+k \sum_{i=1}^{n} H\left(Y_{1^{(1)}}[i] \mid V_{1^{(1)}}[i]\right) \\
& +(k-1) \sum_{i=1}^{n} H\left(Y_{2^{(1)}}[i] \mid V_{2^{(1)}}[i]\right)
\end{aligned}
$$

where step $(a)$ follows from the structure of interference links. That is to say, each copied version of users only imposes interference on the user placed below it. Step $(b)$ follows from $V_{2^{(j)}}[i]=h_{1}\left(X_{1^{(j+1)}}[i], Y_{1^{(j+1)}}[i]\right), V_{1^{(j)}}[i]=$ $h_{2}\left(X_{2^{(j)}}[i], Y_{2^{(j)}}[i]\right)$. Finally, step $(c)$ follows, since the coding schemes of $S_{1^{(j)}}$ S and $S_{2^{(j)}}$ S are the same respectively, $Y_{1^{(j)}}[i] \mathrm{s}$ and $Y_{2^{(j)}}[i] \mathrm{s}$ have the same probability distribution respectively. Furthermore, since $V_{i^{(j)}}=g_{i}\left(X_{i^{(j)}}\right), V_{1^{(j)}}[i] \mathrm{s}$ and $V_{2^{(j)}}[i] \mathrm{s}$ have the same probability distribution respectively.
Proof of part (c) for bound (15).

$$
\begin{aligned}
& H\left(Y_{\Omega_{1}^{c}}^{n}\right)+H\left(Y_{\Omega_{2}^{c}}^{n} \cap \Omega_{1} \mid W_{\mathcal{S} \backslash \Omega_{2}}, Y_{\Omega_{1}^{c}}^{n}\right) \\
& +H\left(Y_{\Omega_{3}^{c}}^{n} \cap \Omega_{2} \mid W_{\mathcal{S} \backslash \Omega_{3}}, Y_{\Omega_{2}^{c}}^{n}\right) \\
& =H\left(Y_{1(2)}^{n}\right)+H\left(Y_{2^{(1)}}^{n} \mid W_{1^{(2)}}, Y_{1(2)}^{n}\right) \\
& +H\left(Y_{1^{(1)}}^{n} \mid W_{2^{(1)}}, W_{1^{(2)}}, Y_{2^{(1)}}^{n}, Y_{1^{(2)}}^{n}\right) \\
& =H\left(Y_{1^{(2)}}^{n}\right)+H\left(Y_{2^{(1)}}^{n} \mid W_{1^{(2)}}, Y_{1(2)}^{n}, X_{1^{(2)}}^{n}\right) \\
& +H\left(Y_{1(1)}^{n} \mid W_{2^{(1)}}, W_{1(2)}, Y_{2^{(1)}}^{n}, Y_{1^{(2)}}^{n}, X_{2^{(1)}}^{n}, X_{1^{(2)}}^{n}\right) \\
& \leq H\left(Y_{1^{(2)}}^{n}\right)+H\left(Y_{2^{(1)}}^{n} \mid Y_{1^{(2)}}^{n}, X_{1^{(2)}}^{n}\right)+H\left(Y_{1^{(1)}}^{n} \mid Y_{2^{(1)}}^{n}, X_{2^{(1)}}^{n}\right) \\
& =\sum_{i=1}^{n} H\left(Y_{1(2)}[i] \mid Y_{1(2)}^{i-1}\right) \\
& +\sum_{i=1}^{n} H\left(Y_{2^{(1)}}[i] \mid Y_{2^{(1)}}^{i-1}, Y_{1^{(2)}}^{n}, X_{1^{(2)}}^{n}\right) \\
& +\sum_{i=1}^{n} H\left(Y_{1^{(1)}}[i] \mid Y_{1^{(1)}}^{i-1}, Y_{2^{(1)}}^{n}, X_{2^{(1)}}^{n}\right) \\
& \stackrel{(a)}{\leq} \sum_{i=1}^{n} H\left(Y_{1^{(2)}}[i]\right)+\sum_{i=1}^{n} H\left(Y_{2^{(1)}}[i] \mid V_{2^{(1)}}[i]\right) \\
& +\sum_{i=1}^{n} H\left(Y_{1^{(1)}}[i] \mid V_{2^{(1)}}[i], V_{1^{(1)}}[i]\right) \\
& \stackrel{(b)}{=} \sum_{i=1}^{n} H\left(Y_{1^{(1)}}[i]\right)+\sum_{i=1}^{n} H\left(Y_{2^{(1)}}[i] \mid V_{2^{(1)}}[i]\right) \\
& +\sum_{i=1}^{n} H\left(Y_{1^{(1)}}[i] \mid V_{2^{(1)}}[i], V_{1^{(1)}}[i]\right)
\end{aligned}
$$

where step $(a)$ follows from $V_{2^{(1)}}[i]=h_{1}\left(X_{1^{(2)}}[i], Y_{1^{(2)}}[i]\right)$, $V_{1^{(1)}}[i]=h_{2}\left(X_{2^{(1)}}[i], Y_{2^{(1)}}[i]\right)$, and $V_{2^{(1)}}[i]=g_{2}\left(X_{2^{(1)}}[i]\right)$. Step (b) follows, since the coding schemes of $S_{1^{(2)}}$ and $S_{1^{(1)}}$ are the same, $Y_{1^{(2)}}[i]$ has the same probability distribution as $Y_{1(1)}[i]$.

\section{Appendix B \\ Proof of part (b) for bound (19).}

$$
\begin{aligned}
& H\left(Y_{\Omega_{1}^{c}}^{n}\right)+\sum_{l=2}^{k+2} H\left(Y_{\Omega_{l}^{c} \cap \Omega_{l-1}}^{n} \mid W_{\mathcal{S} \backslash \Omega_{l}}, Y_{\Omega_{l-1}^{c}}^{n}\right) \\
& \leq \sum_{j=2}^{k} H\left(Y_{1^{(j)}}^{n} \mid Y_{1^{(j+1)}}^{n} X_{1^{(j+1)}}^{n}\right)+C \\
& \leq \sum_{j=2}^{k} \sum_{i=1}^{n} H\left(Y_{1^{(j)}}[i] \mid Y_{1^{(j+1)}}[i] X_{1^{(j+1)}}[i]\right)+C \\
& \stackrel{(a)}{\leq} \sum_{j=2}^{k} \sum_{i=1}^{n} H\left(Y_{1^{(j)}}[i] \mid V_{2^{(1)}}[i] V_{3^{(1)}}[i]\right)+C \\
& \stackrel{(b)}{\leq} k \sum_{i=1}^{n} H\left(Y_{1^{(1)}}[i] \mid V_{2^{(1)}}[i] V_{3^{(1)}}[i]\right)+C
\end{aligned}
$$

where $C \triangleq H\left(Y_{1^{(1)}}^{n}\right)+H\left(Y_{2^{(1)}}^{n}\right)+H\left(Y_{3^{(1)}}^{n}\right)$ and $(a)$ follows from $\left(V_{2^{(1)}}[i], V_{3^{(1)}}[i]\right)=h_{1}\left(X_{1^{(j)}}[i], Y_{1^{(j)}}[i]\right)$. Step $(b)$ follows, since the coding schemes of $S_{1^{(j)}}$ are the same, $Y_{1^{(j)}}[i] \mathrm{s}$ have the same probability distribution. 
Proof of part (b) for bound (20).

$$
\begin{aligned}
& H\left(Y_{\Omega_{1}^{c}}^{n}\right)+\sum_{l=2}^{2 k+2} H\left(Y_{\Omega_{l}^{c}}^{n} \cap \Omega_{l-1} \mid W_{\mathcal{S} \backslash \Omega_{l}}, Y_{\Omega_{l-1}^{c}}^{n}\right) \\
& \leq \sum_{j=1}^{k} H\left(Y_{2^{(j)}}^{n} \mid Y_{1^{(j+1)}}^{n} X_{1^{(j+1)}}^{n}\right) \\
& +\sum_{j=1}^{k} H\left(Y_{1^{(j)}}^{n} \mid Y_{2^{(j)}}^{n} X_{2^{(j)}}^{n}\right)+C \\
& \leq \sum_{j=1}^{k} \sum_{i=1}^{n} H\left(Y_{2^{(j)}}[i] \mid Y_{1^{(j+1)}}[i] X_{1^{(j+1)}}[i]\right) \\
& +\sum_{j=1}^{k} \sum_{i=1}^{n} H\left(Y_{1^{(j)}}[i] \mid Y_{2^{(j)}}[i] X_{2^{(j)}}[i]\right)+C \\
& \stackrel{(a)}{\leq} \sum_{j=1}^{k} \sum_{i=1}^{n} H\left(Y_{2^{(j)}}[i] \mid V_{3^{(1)}}[i]\right)+H\left(Y_{1^{(j)}}[i] \mid V_{1^{(j)}}[i] V_{2^{(j)}}[i] V_{3^{(1)}}[i]\right) \\
& +C \\
& \stackrel{(b)}{\leq} \sum_{i=1}^{n} k H\left(Y_{2^{(1)}}[i] \mid V_{3^{(1)}}[i]\right)+k H\left(Y_{1^{(1)}}[i] \mid V_{1^{(1)}}[i] V_{2^{(1)}}[i] V_{3^{(1)}}[i]\right) \\
& +C
\end{aligned}
$$

where $(a)$ follows from $\left(V_{2^{(j)}}[i], V_{3^{(1)}}[i]\right)=$ $h_{1}\left(X_{1^{(j)}}[i], Y_{1^{(j)}}[i]\right), \quad\left(V_{1^{(j)}}[i], V_{3^{(1)}}[i]\right)$
$h_{2}\left(X_{2^{(j)}}[i], Y_{2(j)}[i]\right)$, and $V_{2^{(j)}}[i]=$
$(b]$ (b) follows, since the coding schemes of $S_{1(j)} \mathrm{S}$ and $S_{2^{(j)}} \mathrm{S}$ are the same respectively, $Y_{1^{(j)}}[i] \mathrm{s}$ and $Y_{2^{(j)}}[i] \mathrm{s}$ have the same probability distribution respectively.

Proof of part (b) for bound (21).

$$
\begin{aligned}
& H\left(Y_{\Omega_{1}^{c}}^{n}\right)+\sum_{l=2}^{2 k+2} H\left(Y_{\Omega_{l}^{c}}^{n} \cap \Omega_{l-1} \mid W_{\mathcal{S} \backslash \Omega_{l}}, Y_{\Omega_{l-1}^{c}}^{n}\right) \\
& \leq \sum_{j=1}^{k} H\left(Y_{2^{(j)}}^{n} \mid Y_{1^{(j+1)}}^{n} X_{1^{(j+1)}}^{n}\right) \\
& +\sum_{j=1}^{k} H\left(Y_{1^{(j)}}^{n} \mid Y_{2^{(j)}}^{n} X_{2^{(j)}}^{n}\right)+C \\
& \leq \sum_{j=1}^{k} \sum_{i=1}^{n} H\left(Y_{2^{(j)}}[i] \mid Y_{1^{(j+1)}}[i] X_{1^{(j+1)}}[i]\right) \\
& +\sum_{j=1}^{k} \sum_{i=1}^{n} H\left(Y_{1^{(j)}}[i] \mid Y_{2^{(j)}}[i] X_{2^{(j)}}[i]\right)+C \\
& \stackrel{(a)}{\leq} \sum_{j=1}^{k} \sum_{i=1}^{n} H\left(Y_{2^{(j)}}[i] \mid V_{2^{(j)}}[i] V_{3^{(1)}}[i]\right)+H\left(Y_{1^{(j)}}[i] \mid V_{1^{(j)}}[i] V_{3^{(1)}}[i]\right) \\
& +C \\
& \stackrel{(b)}{\leq} \sum_{i=1}^{n} k H\left(Y_{2^{(1)}}[i] \mid Y_{2^{(1)}}[i] V_{3^{(1)}}[i]\right)+k H\left(Y_{1^{(1)}}[i] \mid V_{1^{(1)}}[i] V_{3^{(1)}}[i]\right) \\
& +C
\end{aligned}
$$

where step $(a)$ follows from $\left(V_{2(j)}[i], V_{3(1)}[i]\right) \stackrel{(80)}{=}$ $h_{1}\left(X_{1^{(j+1)}}[i], Y_{1^{(j+1)}}[i]\right)$ and $\left(V_{1^{(j)}}[i], V_{3^{(1)}}[i]\right)=$ $h_{2}\left(X_{2^{(j)}}[i], Y_{2^{(j)}}[i]\right)$. Step $(b)$ follows, since the coding schemes of $S_{1^{(j)} \mathrm{S}}$ and $S_{2^{(j)} \mathrm{S}}$ are the same respectively, $Y_{1^{(j)}}[i] \mathrm{s}$ and $Y_{2^{(j)}}[i] \mathrm{s}$ have the same probability distribution respectively.
Proof of part (b) for bound (22).

$$
\begin{aligned}
& H\left(Y_{\Omega_{1}^{c}}^{n}\right)+\sum_{l=2}^{3 k+2} H\left(Y_{\Omega_{l}^{c} \cap \Omega_{l-1}}^{n} \mid W_{\mathcal{S} \backslash \Omega_{l}}, Y_{\Omega_{l-1}^{c}}^{n}\right) \\
& \leq \sum_{j} H\left(Y_{1^{(2 j+1)}}^{n} \mid Y_{1^{(2 j+2)}}^{n} X_{1^{(2 j+2)}}^{n}\right) \\
& +\sum_{j} H\left(Y_{2^{(j)}}^{n} \mid Y_{1^{(2 j+1)}}^{n} X_{1^{(2 j+1)}}^{n}\right) \\
& +\sum_{j} H\left(Y_{1^{(2 j)}}^{n} \mid Y_{2^{(j)}}^{n} X_{2^{(j)}}^{n}\right)+C \\
& \leq \sum_{j} \sum_{i=1}^{n} H\left(Y_{1^{(2 j+1)}}[i] \mid Y_{1^{(2 j+2)}}[i] X_{1^{(2 j+2)}}[i]\right) \\
& +\sum_{j} \sum_{i=1}^{n} H\left(Y_{2^{(j)}}[i] \mid Y_{1^{(2 j+1)}}[i] X_{1^{(2 j+1)}}[i]\right) \\
& +\sum_{j=1} \sum_{i=1}^{n} H\left(Y_{1^{(2 j)}}[i] \mid Y_{2^{(j)}}[i] X_{2^{(j)}}[i]\right)+C \\
& \quad \stackrel{(a)}{\leq} \sum_{j} \sum_{i=1}^{n} H\left(Y_{1^{(2 j+1)}}[i] \mid V_{3^{(1)}}[i]\right) \\
& +\sum_{j} \sum_{i=1}^{n} H\left(Y_{2^{(j)}}[i] \mid V_{2^{(j)}}[i] V_{3^{(1)}}[i]\right) \\
& +\sum_{j} \sum_{i=1}^{n} H\left(Y_{1^{(2 j)}}[i] \mid V_{1^{(2 j)}}[i] V_{2^{(j)}}[i] V_{3^{(1)}}[i]\right)+C \\
& +(b) \\
& \leq \sum_{i=1}^{n} k H\left(Y_{1^{(1)}}[i] \mid V_{3^{(1)}}[i]\right) \\
& +\sum_{i=1}^{n} k H\left(Y_{2^{(1)}}[i] \mid V_{2^{(1)}}[i] V_{3^{(1)}}[i]\right) \\
& +\sum_{i=1}^{n} k H\left(Y_{1^{(1)}}[i] \mid V_{1^{(1)}}[i] V_{2^{(1)}}[i] V_{3^{(1)}}[i]\right)+C \\
&
\end{aligned}
$$

where step $(a)$ follows from $\left(V_{2^{(j+1)}}[i], V_{3^{(1)}}[i]\right)=$ $h_{1}\left(X_{1^{(2 j+2)}}[i], Y_{1^{(2 j+2)}}[i]\right), \quad\left(V_{2^{(j)}}[i], V_{3^{(1)}}[i]\right)=$ $h_{1}\left(X_{1^{(2 j+1)}}[i], Y_{1^{(2 j+1)}}[i]\right), \quad\left(V_{1^{(2 j)}}[i], V_{3^{(1)}}[i]\right) \quad=$ $h_{2}\left(X_{2^{(j)}}[i], Y_{2^{(j)}}[i]\right)$, and $V_{2^{(j)}}[i]=g_{2}\left(X_{2^{(j)}}[i]\right)$. Step (b) follows, since the coding schemes of $S_{1^{(j)}} \mathrm{s}$ and $S_{2^{(j)} \mathrm{S}}$ are the same respectively, $Y_{1^{(j)}}[i] \mathrm{s}$ and $Y_{2^{(j)}}[i] \mathrm{s}$ have the same probability distribution respectively.

Proof of part (b) for bound (23).

$$
\begin{aligned}
& H\left(Y_{\Omega_{1}^{c}}^{n}\right)+\sum_{l=2}^{3 k+2} H\left(Y_{\Omega_{l}^{c}}^{n} \cap \Omega_{l-1} \mid W_{\mathcal{S} \backslash \Omega_{l}}, Y_{\Omega_{l-1}^{c}}^{n}\right) \\
& \leq \sum_{j} H\left(Y_{3^{(j)}}^{n} \mid Y_{1^{(j+1)}}^{n} X_{1^{(j+1)}}^{n} X_{2^{(j+1)}}^{n}\right) \\
& +\sum_{j} H\left(Y_{2^{(j)}}^{n} \mid Y_{1^{(j+1)}}^{n} X_{1^{(j+1)}}^{n} X_{3^{(j)}}^{n}\right) \\
& +\sum_{j} H\left(Y_{1^{(j)}}^{n} \mid Y_{2^{(j)}}^{n} X_{2^{(j)}}^{n}\right) \\
& \leq \sum_{j=1} \sum_{i=1}^{n} H\left(Y_{3^{(j)}}[i] \mid Y_{1^{(j+1)}}[i] X_{1^{(j+1)}}[i] X_{2^{(j+1)}}[i]\right)
\end{aligned}
$$


$+\sum_{j=1} \sum_{i=1}^{n} H\left(Y_{2^{(j)}}[i] \mid Y_{1^{(j+1)}}[i] X_{1^{(j+1)}}[i] X_{3^{(j)}}[i]\right)$

$+\sum_{j=1} \sum_{i=1}^{n} H\left(Y_{1^{(j)}}[i] \mid Y_{2^{(j)}}[i] X_{2^{(j)}}[i]\right)$

$\stackrel{(a)}{\leq} \sum_{j} \sum_{i=1}^{n} H\left(Y_{3^{(j)}}[i] \mid V_{1^{(j+1)}}[i] V_{2^{(j+1)}}[i] V_{3^{(j)}}[i]\right)$

$+\sum_{j} \sum_{i=1}^{n} H\left(Y_{2^{(j)}}[i] \mid V_{2^{(j)}}[i] V_{3^{(j)}}[i]\right)+H\left(Y_{1^{(j)}}[i] \mid V_{1^{(j)}}[i]\right)+C$

$\stackrel{(b)}{\leq} \sum_{i=1}^{n} k H\left(Y_{3^{(1)}}[i] \mid V_{1^{(1)}}[i], V_{2^{(1)}}[i], V_{3^{(1)}}[i]\right)$

$+\sum_{i=1}^{n} k H\left(Y_{2^{(1)}}[i] \mid V_{2^{(1)}}[i], V_{3^{(1)}}[i]\right)+k H\left(Y_{1^{(1)}}[i] \mid V_{1^{(1)}}[i]\right)+C$

where step $(a)$ follows from $\left(V_{2^{(j)}}[i], V_{3^{(j)}}[i]\right)=$ $h_{1}\left(X_{1^{(j+1)}}[i], Y_{1^{(j+1)}}[i]\right), \quad\left(V_{1^{(j)}}[i], V_{3^{(j)}}[i]\right)=$ $h_{2}\left(X_{2^{(j)}}[i], Y_{2^{(j)}}[i]\right), \quad V_{3^{(j)}}[i]=g_{3}\left(X_{3^{(j)}}[i]\right)$, $V_{1^{(j+1)}}[i]=g_{1}\left(X_{1^{(j+1)}}[i]\right)$, and $V_{2^{(j+1)}}[i]=g_{2}\left(X_{2^{(j+1)}}[i]\right)$. Step $(b)$ follows, since the coding schemes of $S_{1^{(j)}} \mathrm{s}, S_{2^{(j)}} \mathrm{s}$, and $S_{3^{(j)}} \mathrm{s}$ are the same respectively, $Y_{1^{(j)}}[i] \mathrm{s}, Y_{2^{(j)}}[i] \mathrm{s}$, and $Y_{3^{(j)}}[i]$ s have the same probability distribution respectively.

Proof of part (b) for bound (24).

$$
\begin{aligned}
& H\left(Y_{\Omega_{1}^{c}}^{n}\right)+\sum_{l=2}^{3 k+2} H\left(Y_{\Omega_{l}^{c} \cap \Omega_{l-1}}^{n} \mid W_{\mathcal{S} \backslash \Omega_{l}}, Y_{\Omega_{l-1}^{c}}^{n}\right) \\
& \leq \sum_{j=1} H\left(Y_{2^{(j)}}^{n} \mid Y_{1^{(j+1)}}^{n}, X_{1^{(j+1)}}^{n}\right)+H\left(Y_{3^{(j)}}^{n} \mid Y_{2^{(j)}}^{n}, X_{2^{(j)}}^{n}\right) \\
& +\sum_{j=1} H\left(Y_{1^{(j)}}^{n} \mid Y_{3^{(j)}}^{n}, X_{3^{(j)}}^{n}\right)+C \\
& \leq \sum_{j} \sum_{i=1}^{n} H\left(Y_{2^{(j)}}[i] \mid Y_{1^{(j+1)}}[i], X_{1^{(j+1)}}[i]\right) \\
& +\sum_{j} \sum_{i=1}^{n} H\left(Y_{3^{(j)}}[i] \mid Y_{2^{(j)}}[i], X_{2^{(j)}}[i]\right) \\
& +\sum_{j} \sum_{i=1}^{n} H\left(Y_{1^{(j)}}[i] \mid Y_{3^{(j)}}[i], X_{3^{(j)}}[i]\right)+C \\
& \stackrel{(a)}{\leq} \sum_{j} \sum_{i=1}^{n} H\left(Y_{2^{(j)}}[i] \mid V_{2^{(j)}}[i], V_{1^{(j+1)}}[i]\right) \\
& +\sum_{j} \sum_{i=1}^{n} H\left(Y_{3^{(j)}}[i] \mid V_{3^{(j)}}[i], V_{2^{(j)}}[i]\right)+H\left(Y_{1^{(j)}}[i] \mid V_{1^{(j)}}[i], V_{3^{(j)}}[i]\right) \\
& +C \\
& \stackrel{(b)}{\leq} \sum_{i=1}^{n} k H\left(Y_{3^{(1)}}[i] \mid V_{2^{(1)}}[i], V_{3^{(1)}}[i]\right)+k H\left(Y_{2^{(1)}}[i] \mid V_{2^{(1)}}[i], V_{1^{(1)}}[i]\right) \\
& +\sum_{i=1}^{n} k H\left(Y_{1^{(1)}}[i] \mid V_{1^{(1)}}[i], V_{3^{(1)}}[i]\right)+C
\end{aligned}
$$

where step $(a)$ follows from $\left(V_{2(j)}[i], V_{3}(j+1)[i]\right) \stackrel{(83)}{=}$ $h_{1}\left(X_{1^{(j+1)}}[i], Y_{1^{(j+1)}}[i]\right), \quad\left(V_{1^{(j+1)}}[i], V_{3(j)}[i]\right)=$ $h_{2}\left(X_{2^{(j)}}[i], Y_{2^{(j)}}[i]\right), \quad\left(V_{1^{(j)}}[i], V_{2^{(j)}}[i]\right)$
$h_{3}\left(X_{3^{(j)}}[i], Y_{3^{(j)}}\right)$ $V_{1^{(j+1)}}[i]=g_{1}\left(X_{1^{(j+1)}}[i]\right)$, and $V_{2^{(j)}}[i]=g_{2}\left(X_{2^{(j)}}[i]\right)$. Step $(b)$ follows, since the coding schemes of $S_{1^{(j)}} \mathrm{s}, S_{2^{(j)}} \mathrm{s}$, and $S_{3(j)}$ s are the same respectively, $Y_{1^{(j)}}[i] \mathrm{s}, Y_{2^{(j)}}[i] \mathrm{s}$, and $Y_{3^{(j)}}[i]$ s have the same probability distribution respectively. Proof of part (b) for bound (25.

$$
\begin{aligned}
& H\left(Y_{\Omega_{1}^{c}}^{n}\right)+\sum_{l=2}^{3 k+2} H\left(Y_{\Omega_{l}^{c} \cap \Omega_{l-1}}^{n} \mid W_{\mathcal{S} \backslash \Omega_{l}}, Y_{\Omega_{l^{c}}^{c}}^{n}\right) \\
& \leq \sum_{j} H\left(Y_{2^{(j)}}^{n} \mid Y_{1^{(j+1)}}^{n} X_{1^{(j+1)}}^{n}\right)+H\left(Y_{3^{(j)}}^{n} \mid Y_{2^{(j)}}^{n} X_{2^{(j)}}^{n}\right) \\
& +\sum_{j} H\left(Y_{1^{(j)}}^{n} \mid Y_{3^{(j)}}^{n} X_{3^{(j)}}^{n} X_{2^{(j)}}^{n}\right) \\
& \leq \sum_{j} \sum_{i=1}^{n} H\left(Y_{2^{(j)}}[i] \mid Y_{1^{(j+1)}}[i] X_{1^{(j+1)}}[i]\right) \\
& +\sum_{j} \sum_{i=1}^{n} H\left(Y_{3^{(j)}}[i] \mid Y_{2^{(j)}}[i] X_{2^{(j)}}[i]\right) \\
& +\sum_{j=1} \sum_{i=1}^{n} H\left(Y_{1^{(j)}}[i] \mid Y_{3^{(j)}}[i] X_{3^{(j)}}[i] X_{2^{(j)}}[i]\right) \\
& \quad \stackrel{(a)}{\leq} \sum_{j} \sum_{i=1}^{n} H\left(Y_{2^{(j)}}[i] \mid V_{1^{(j+1)}}[i]\right)+H\left(Y_{3^{(j)}}[i] \mid V_{2^{(j)}}[i] V_{3^{(j)}}[i]\right) \\
& +\sum_{j} \sum_{i=1}^{n} H\left(Y_{1^{(j)}}[i] \mid V_{1^{(j)}}[i] V_{2^{(j)}}[i] V_{3^{(j)}}[i]\right)+C \\
& \quad(b) \\
& \leq \sum_{i=1}^{n} k H\left(Y_{2^{(1)}}[i] \mid V_{1^{(1)}}[i]\right)+k H\left(Y_{3^{(1)}}[i] \mid V_{2^{(1)}}[i] V_{3^{(1)}}[i]\right) \\
& +\sum_{i=1}^{n} k H\left(Y_{1^{(1)}}[i] \mid V_{1^{(1)}}[i] V_{2^{(1)}}[i] V_{3^{(1)}}[i]\right)+C
\end{aligned}
$$

where step $(a)$ follows from $\left(V_{1^{(j+1)}}[i], V_{3^{(j)}}[i]\right)=$ $h_{2}\left(X_{2^{(j)}}[i], Y_{2^{(j)}}[i]\right), \quad\left(V_{1^{(j)}}[i], V_{2^{(j)}}[i]\right)=$ $h_{3}\left(X_{3^{(j)}}[i], Y_{3^{(j)}}[i]\right), \quad V_{3^{(j)}}[i]=g_{3}\left(X_{3^{(j)}}[i]\right)$, $V_{1^{(j+1)}}[i]=g_{1}\left(X_{1^{(j+1)}}[i]\right)$, and $V_{2^{(j)}}[i]=g_{2}\left(X_{2^{(j)}}[i]\right)$. Step (b) follows, since the coding schemes of $S_{1^{(j)}} \mathrm{s}, S_{2^{(j)}} \mathrm{S}$, and $S_{3^{(j)}} \mathrm{s}$ are the same respectively, $Y_{1^{(j)}}[i] \mathrm{s}, Y_{2^{(j)}}[i] \mathrm{s}$, and $Y_{3^{(j)}}[i] \mathrm{s}$ have the same probability distribution respectively.

\section{Proof of part (b) for bound (26).}

$$
\begin{aligned}
& H\left(Y_{\Omega_{1}^{c}}^{n}\right)+\sum_{l=2}^{3} H\left(Y_{\Omega_{l}^{c} \cap \Omega_{l-1}}^{n} \mid W_{\mathcal{S} \backslash \Omega_{l}}, Y_{\Omega_{l-1}^{c}}^{n}\right) \\
& \leq \sum_{j} H\left(Y_{3^{(1)}}^{n}\right)+H\left(Y_{2^{(1)}}^{n} \mid Y_{3^{(1)}}^{n} X_{3^{(1)}}^{n}\right) \\
& +\sum_{j} H\left(Y_{1^{(1)}}^{n} \mid Y_{2^{(1)}}^{n} X_{2^{(1)}}^{n} X_{3^{(1)}}^{n}\right) \\
& \leq \sum_{j} \sum_{i=1}^{n} H\left(Y_{3^{(1)}}[i]\right) \\
& +\sum_{j} \sum_{i=1}^{n} H\left(Y_{2^{(1)}}[i] \mid Y_{3^{(1)}}[i] X_{3^{(1)}}[i]\right) \\
& +\sum_{j=1} \sum_{i=1}^{n} H\left(Y_{1^{(1)}}[i] \mid Y_{2^{(1)}}[i] X_{2^{(1)}}[i] X_{3^{(1)}}[i]\right) \\
& \stackrel{(a)}{\leq} \sum_{j} \sum_{i=1}^{n} H\left(Y_{3^{(1)}}[i]\right)+H\left(Y_{2^{(1)}}[i] \mid V_{1^{(1)}}[i] V_{2^{(1)}}[i] V_{3^{(1)}}[i]\right) \\
& +\sum_{j} \sum_{i=1}^{n} H\left(Y_{1^{(1)}}[i] \mid V_{1^{(1)}}[i] V_{2^{(1)}}[i] V_{3^{(1)}}[i]\right)
\end{aligned}
$$


where step $(a)$ follows from $\left(V_{1^{(1)}}[i], V_{3^{(1)}}[i]\right)=$ $h_{2}\left(X_{2^{(1)}}[i], Y_{2^{(1)}}[i]\right), \quad\left(V_{1^{(1)}}[i], V_{2^{(1)}}[i]\right)=$ $h_{3}\left(X_{3^{(1)}}[i], Y_{3^{(1)}}[i]\right), \quad V_{3^{(1)}}[i]=g_{3}\left(X_{3^{(1)}}[i]\right), \quad$ and $V_{2^{(1)}}[i]=g_{2}\left(X_{2^{(1)}}[i]\right)$.

Proof of part (b) for bound (27).

$$
\begin{aligned}
H & \left(Y_{\Omega_{1}^{c}}^{n}\right)+H\left(Y_{\Omega_{2}^{c}}^{n} \cap \Omega_{1} \mid W_{\mathcal{S} \backslash \Omega_{2}}, Y_{\Omega_{1}^{c}}^{n}\right) \\
+ & H\left(Y_{\Omega_{3}^{c} \cap \Omega_{2}}^{n} \mid W_{\mathcal{S} \backslash \Omega_{3}}, Y_{\Omega_{2}^{c}}^{n}\right)+H\left(Y_{\Omega_{4}^{c}}^{n} \cap \Omega_{3} \mid W_{\mathcal{S} \backslash \Omega_{4}}, Y_{\Omega_{3}^{c}}^{n}\right) \\
= & H\left(Y_{1^{(2)}}^{n}\right)+H\left(Y_{3^{(1)}}^{n} \mid W_{1^{(2)}}, Y_{1^{(2)}}^{n}\right) \\
+ & H\left(Y_{2^{(1)}}^{n} \mid W_{3^{(1)}}, W_{1^{(2)}}, Y_{3^{(1)}}^{n}, Y_{1^{(2)}}^{n}\right) \\
+ & H\left(Y_{1^{(1)}}^{n} \mid W_{2^{(1)}}, W_{3^{(1)}}, W_{1^{(2)}}, Y_{2^{(1)}}^{n}, Y_{3^{(1)}}^{n}, Y_{1^{(2)}}^{n}\right) \\
= & H\left(Y_{1^{(2)}}^{n}\right)+H\left(Y_{3^{(1)}}^{n} \mid W_{1^{(2)}}, Y_{1^{(2)}}^{n}, X_{1^{(2)}}^{n}\right) \\
+ & H\left(Y_{2^{(1)}}^{n} \mid W_{3^{(1)}}, W_{1^{(2)}}, Y_{3^{(1)}}^{n}, Y_{1^{(2)}}^{n}, X_{3^{(1)}}^{n}, X_{\left.1^{(2)}\right)}^{n}\right) \\
+ & H\left(Y_{1^{(1)}}^{n} \mid W_{2^{(1)}}, W_{3^{(1)}}, W_{1^{(2)}}, Y_{2^{(1)}}^{n}, Y_{3^{(1)}}^{n}, Y_{1^{(2)}}^{n}, X_{2^{(1)}}^{n}, X_{3^{(1)}}^{n}, X_{1^{(2)}}^{n}\right) \\
\leq & H\left(Y_{1^{(2)}}^{n}\right)+H\left(Y_{3^{(1)}}^{n} \mid Y_{\left.1^{(2)}\right)}^{n}, X_{1^{(2)}}^{n}\right) \\
+ & H\left(Y_{2^{(1)}}^{n} \mid Y_{3^{(1)}}^{n}, X_{3^{(1)}}^{n}, Y_{1^{(2)}}^{n}, X_{1^{(2)}}^{n}\right) \\
+ & H\left(Y_{1^{(1)}}^{n} \mid Y_{2^{(1)}}^{n}, X_{2^{(1)}}^{n}, Y_{3^{(1)}}^{n}, X_{3^{(1)}}^{n}, Y_{1^{(2)}}^{n}, X_{1^{(2)}}^{n}\right) \\
= & \sum_{i} H\left(Y_{1^{(2)}}[i] \mid Y_{1^{(2)}}^{i-1}\right)+\sum_{i} H\left(Y_{3^{(1)}}[i] \mid Y_{3^{(1)}}^{i-1}, Y_{1^{(2)}}^{n}, X_{1^{(2)}}^{n}\right) \\
& +\sum_{i} H\left(Y_{2^{(1)}}[i] \mid Y_{2^{(1)}}^{i-1}, Y_{3^{(1)}}^{n}, X_{3^{(1)}}^{n}, Y_{1^{(2)}}^{n}, X_{1^{(2)}}^{n}\right) \\
& +\sum_{i} H\left(Y_{1^{(1)}}[i] \mid Y_{1^{(1)}}^{i-1}, Y_{2^{(1)}}^{n}, X_{2^{(1)}}^{n}, Y_{3^{(1)}}^{n}, X_{3^{(1)}}^{n}, Y_{1^{(2)}}^{n}, X_{1^{(2)}}^{n}\right) \\
&
\end{aligned}
$$$$
\stackrel{(a)}{\leq} \sum_{i} H\left(Y_{1^{(2)}}[i]\right)+\sum_{i} H\left(Y_{3^{(1)}}[i] \mid V_{1^{(2)}}[i], V_{2^{(1)}}[i], V_{3^{(1)}}[i]\right)
$$$$
+\sum_{i} H\left(Y_{2^{(1)}}[i] \mid V_{2^{(1)}}[i], V_{3^{(1)}}[i]\right)
$$$$
+\sum_{i} H\left(Y_{1^{(1)}}[i] \mid V_{1^{(1)}}[i], V_{2^{(1)}}[i], V_{3^{(1)}}[i]\right)
$$$$
\stackrel{(b)}{\leq} \sum_{i} H\left(Y_{1^{(1)}}[i]\right)+\sum_{i} H\left(Y_{3^{(1)}}[i] \mid V_{1^{(1)}}[i], V_{2^{(1)}}[i], V_{3^{(1)}}[i]\right)
$$$$
+\sum_{i} H\left(Y_{2^{(1)}}[i] \mid V_{2^{(1)}}[i], V_{3^{(1)}}[i]\right)
$$$$
+\sum_{i} H\left(Y_{1^{(1)}}[i] \mid V_{1^{(1)}}[i], V_{2^{(1)}}[i], V_{3^{(1)}}[i]\right)
$$

where $(a)$ follows from $V_{1^{(2)}}[i]=g_{1}\left(X_{1^{(2)}}[i]\right)$, $\left(V_{2^{(1)}}[i], V_{3^{(1)}}[i]\right)=h_{1}\left(X_{1^{(2)}}[i], Y_{1^{(2)}}[i]\right), \quad V_{3^{(1)}}[i]=$ $g_{3}\left(X_{3^{(1)}}[i]\right), V_{2^{(1)}}[i]=g_{2}\left(X_{2^{(1)}}[i]\right)$, and $\left(V_{1^{(1)}}[i], V_{3^{(1)}}[i]\right)=$ $h_{2}\left(X_{2^{(1)}}[i], Y_{2^{(1)}}[i]\right)$. Step $(b)$ follows, since the coding schemes of $S_{1^{(2)}}$ is the same as $S_{1^{(1)}}, Y_{1^{(2)}}[i]$ and $Y_{1^{(1)}}[i]$ have the same probability distribution. It should be noted that $V_{1^{(2)}}[i]$ and $V_{1^{(1)}}[i]$ have the same probability distribution as well.

\section{Proof of part (b) for bound 28.}

$$
\begin{aligned}
& H\left(Y_{\Omega_{1}^{c}}^{n}\right)+\sum_{l=2}^{4 k+2} H\left(Y_{\Omega_{l}^{c} \cap \Omega_{l-1}}^{n} \mid W_{\mathcal{S} \backslash \Omega_{l}}, Y_{\Omega_{l-1}^{c}}^{n}\right) \\
& \leq \sum_{j} H\left(Y_{1^{(2 j+1)}}^{n} \mid Y_{3^{(j+1)}}^{n}, X_{3^{(j+1)}}^{n}\right)+H\left(Y_{3^{(j)}}^{n} \mid Y_{1^{(2 j+1)}}^{n}, X_{1^{(2 j+1)}}^{n}\right) \\
& +\sum_{j} H\left(Y_{2^{(j)}}^{n} \mid Y_{3^{(j)}}^{n}, X_{3^{(j)}}^{n}\right)+H\left(Y_{1^{(2 j)}}^{n} \mid Y_{2^{(j)}}^{n}, X_{2^{(j)}}^{n}, X_{3^{(j)}}^{n}\right)+C
\end{aligned}
$$

$\leq \sum_{j} \sum_{i=1}^{n} H\left(Y_{1^{(2 j+1)}}[i] \mid Y_{3^{(j+1)}}[i], X_{3^{(j+1)}}[i]\right)$

$+\sum_{j} \sum_{i=1}^{n} H\left(Y_{3^{(j)}}[i] \mid Y_{1^{(2 j+1)}}[i], X_{1^{(2 j+1)}}[i]\right)$

$+\sum_{j} \sum_{i=1}^{n} H\left(Y_{2^{(j)}}[i] \mid Y_{3^{(j)}}[i], X_{3^{(j)}}[i]\right)$

$+\sum_{j} \sum_{i=1}^{n} H\left(Y_{1^{(2 j)}}[i] \mid Y_{2^{(j)}}[i], X_{2^{(j)}}[i], X_{3^{(j)}}[i]\right)+C$

$\stackrel{(a)}{\leq} \sum_{j=1} \sum_{i=1}^{n} H\left(Y_{1^{(2 j+1)}}[i] \mid V_{1^{(2 j+1)}}[i]\right)+H\left(Y_{3^{(j)}}[i] \mid V_{2^{(j)}}[i], V_{3^{(j)}}[i]\right)$

$+\sum_{j=1} \sum_{i=1}^{n} H\left(Y_{2^{(j)}}[i] \mid V_{2^{(j)}}[i], V_{3^{(j)}}[i]\right)$

$+\sum_{j=1} \sum_{i=1}^{n} H\left(Y_{1^{(2 j)}}[i] \mid V_{1^{(2 j)}}[i], V_{2^{(j)}}[i], V_{3^{(j)}}[i]\right)+C$

$\stackrel{(b)}{\leq} \sum_{i=1}^{n} k H\left(Y_{1^{(1)}}[i] \mid V_{1^{(1)}}[i]\right)+k H\left(Y_{3^{(1)}}[i] \mid V_{2^{(1)}}[i], V_{3^{(1)}}[i]\right)$

$+\sum_{i=1}^{n} k H\left(Y_{2^{(1)}}[i] \mid V_{2^{(1)}}[i], V_{3^{(1)}}[i]\right)$

$+\sum_{i=1}^{n} k H\left(Y_{1^{(1)}}[i] \mid V_{1^{(1)}}[i], V_{2^{(1)}}[i], V_{3^{(1)}}[i]\right)+C$

where step $(a)$ follows from $-\left(V_{2(j)}[i], V_{3(j)}[i]\right)$ (87) where step $(a)$ follows from $\left(V_{2^{(j)}}[i], V_{3^{(j)}}[i]\right)=$ $h_{1}\left(X_{1^{(2 j+1)}}[i], Y_{1^{(2 j+1)}}[i]\right), \quad\left(V_{1^{(2 j)}}[i], V_{3^{(j)}}[i]\right) \quad=$ $h_{2}\left(X_{2^{(j)}}[i], Y_{2^{(j)}}[i]\right), \quad\left(V_{1^{(2 j+1)}}[i], V_{2^{(j+1)}}[i]\right) \quad=$ $h_{3}\left(X_{3^{(j+1)}}[i], Y_{3^{(j+1)}}[i]\right), \quad V_{3^{(j)}}[i]=g_{3}\left(X_{3^{(j)}}[i]\right)$, $V_{1^{(2 j+1)}}[i]=g_{1}\left(X_{1^{(2 j+1)}}[i]\right)$, and $V_{2^{(j)}}[i]=g_{2}\left(X_{2^{(j)}}[i]\right)$. Step (b) follows, since the coding schemes of $S_{1^{(j)}} \mathrm{s}, S_{2^{(j)}} \mathrm{s}$, and $S_{3^{(j)}} \mathrm{S}$ are the same respectively, $Y_{1^{(j)}}[i] \mathrm{s}, Y_{2^{(j)}}[i] \mathrm{s}$, and $Y_{3^{(j)}}[i] \mathrm{s}$ have the same probability distribution respectively.

\section{Proof of part (b) for bound (29).}

$$
\begin{aligned}
& H\left(Y_{\Omega_{1}^{c}}^{n}\right)+\sum_{l=2}^{4 k+2} H\left(Y_{\Omega_{l}^{c} \cap \Omega_{l-1}}^{n} \mid W_{\mathcal{S} \backslash \Omega_{l}}, Y_{\Omega_{l-1}^{c}}^{n}\right) \\
& \leq \sum_{j} H\left(Y_{1^{(2 j+1)}}^{n} \mid X_{2^{(j+1)}}^{n}\right)+H\left(Y_{3^{(j)}}^{n} \mid Y_{1^{(2 j+1)}}^{n}, X_{1^{(2 j+1)}}^{n}, X_{2^{(j+1)}}^{n}\right) \\
& +\sum_{j} H\left(Y_{1^{(2 j)}}^{n} \mid Y_{3^{(j)}}^{n}, X_{3^{(j)}}^{n}\right)+H\left(Y_{2^{(j)}}^{n} \mid Y_{1^{(2 j)}}^{n}, X_{1^{(2 j)}}^{n}, X_{3^{(j)}}^{n}\right) \\
& \leq \sum_{j} \sum_{i=1}^{n} H\left(Y_{1^{(2 j+1)}}[i] \mid X_{2^{(j+1)}}[i]\right) \\
& +\sum_{j} \sum_{i=1}^{n} H\left(Y_{3^{(j)}}[i] \mid Y_{1^{(2 j+1)}}[i], X_{1^{(2 j+1)}}[i], X_{2^{(j+1)}}[i]\right) \\
& +\sum_{j} \sum_{i=1}^{n} H\left(Y_{1^{(2 j)}}[i] \mid Y_{3^{(j)}}[i], X_{3^{(j)}}[i]\right) \\
& +\sum_{j} \sum_{i=1}^{n} H\left(Y_{2^{(j)}}[i] \mid Y_{1^{(2 j)}}[i], X_{1^{(2 j)}}[i], X_{3^{(j)}}[i]\right)
\end{aligned}
$$




$$
\begin{aligned}
& \stackrel{(a)}{\leq} \sum_{j} \sum_{i=1}^{n} H\left(Y_{1^{(2 j+1)}}[i] \mid V_{2^{(j+1)}}[i]\right)+H\left(Y_{3^{(j)}}[i] \mid V_{2^{(j+1)}}[i], V_{3^{(j)}}[i]\right) \\
& +\sum_{j} \sum_{i=1}^{n} H\left(Y_{1^{(2 j)}}[i] \mid V_{1^{(2 j)}}[i], V_{3^{(j)}}[i]\right) \\
& +\sum_{j} \sum_{i=1}^{n} H\left(Y_{2^{(j)}}[i] \mid V_{1^{(2 j)}}[i], V_{2^{(j)}}[i], V_{3^{(j)}}[i]\right)+C \\
& \stackrel{(b)}{\leq} \sum_{i=1}^{n} k H\left(Y_{1^{(1)}}[i] \mid V_{1^{(1)}}[i], V_{3^{(1)}}[i]\right)+k H\left(Y_{3^{(1)}}[i] \mid V_{2^{(1)}}[i], V_{3^{(1)}}[i]\right) \\
& +\sum_{i=1}^{n} k H\left(Y_{2^{(1)}}[i] \mid V_{1^{(1)}}[i], V_{2^{(1)}}[i], V_{3^{(1)}}[i]\right)+k H\left(Y_{1^{(1)}}[i] \mid V_{2^{(1)}}[i]\right) \\
& +C
\end{aligned}
$$

where step $(a)$ follows from $\left(V_{2^{(j+1)}}[i], V_{3^{(j)}}[i]\right)=$ $h_{1}\left(X_{1^{(2 j+1)}}[i], Y_{1^{(2 j+1)}}[i]\right), \quad\left(V_{2^{(j)}}[i], V_{3^{(j)}}[i]\right)=$ $h_{1}\left(X_{1^{(2 j)}}[i], Y_{1^{(2 j)}}[i]\right), \quad\left(V_{1^{(2 j)}}[i], V_{2^{(j+1)}}[i]\right)=$ $h_{3}\left(X_{3^{(j)}}[i], Y_{3^{(j)}}[i]\right), \quad V_{3^{(j)}}[i]=g_{3}\left(X_{3^{(j)}}[i]\right)$, $V_{1(2 j)}[i]=g_{1}\left(X_{1^{(2 j)}}[i]\right)$, and $V_{2^{(j+1)}}[i]=g_{2}\left(X_{2^{(j+1)}}[i]\right)$. Step (b) follows, since the coding schemes of $S_{1^{(j)}} \mathrm{s}, S_{2^{(j)}} \mathrm{s}$, and $S_{3^{(j)}} \mathrm{S}$ are the same respectively, $Y_{1^{(j)}}[i] \mathrm{s}, Y_{2^{(j)}}[i] \mathrm{s}$, and $Y_{3^{(j)}}[i] \mathrm{s}$ have the same probability distribution respectively.

\section{Proof of part (b) for bound (30).}

$$
\begin{aligned}
& H\left(Y_{\Omega_{1}^{c}}^{n}\right)+\sum_{l=2}^{4 k+2} H\left(Y_{\Omega_{l}^{c}}^{n} \cap \Omega_{l-1} \mid W_{\mathcal{S} \backslash \Omega_{l}}, Y_{\Omega_{l-1}^{c}}^{n}\right)+n \epsilon_{n} \\
& \leq \sum_{j} H\left(Y_{1(2 j+1)}^{n} \mid X_{3(j+1)}^{n}\right) \\
& +\sum_{j} H\left(Y_{3^{(j)}}^{n} \mid Y_{1^{(2 j+1)}}^{n}, X_{1^{(2 j+1)}}^{n}, Y_{2^{(j+1)}}^{n}, X_{2^{(j+1)}}^{n}\right) \\
& +\sum_{j} H\left(Y_{2^{(j)}}^{n} \mid Y_{3(j)}^{n}, X_{3(j)}^{n}, X_{1(2 j+1)}^{n}\right) \\
& +\sum_{j} H\left(Y_{1^{(2 j)}}^{n} \mid Y_{3^{(j)}}^{n}, X_{3^{(j)}}^{n}, X_{2^{(j)}}^{n}\right) \\
& \leq \sum_{j} \sum_{i=1}^{n} H\left(Y_{1(2 j+1)}[i] \mid X_{3(j+1)}[i]\right) \\
& +\sum_{j} \sum_{i=1}^{n} H\left(Y_{3(j)}[i] \mid Y_{1^{(2 j+1)}}[i], X_{1^{(2 j+1)}}[i], Y_{2^{(j+1)}}[i], X_{\left.2^{(j+1)}[i]\right)}\right. \\
& +\sum_{j} \sum_{i=1}^{n} H\left(Y_{2^{(j)}}[i] \mid Y_{3^{(j)}}[i], X_{3^{(j)}}[i], X_{1^{(2 j+1)}}[i]\right) \\
& +\sum_{j} \sum_{i=1}^{n} H\left(Y_{1^{(2 j)}}[i] \mid Y_{3^{(j)}}[i], X_{3^{(j)}}[i], X_{2^{(j)}}[i]\right) \\
& \stackrel{(a)}{\leq} \sum_{j} \sum_{i=1}^{n} H\left(Y_{1^{(2 j+1)}}[i] \mid V_{3^{(j+1)}}[i]\right)+H\left(Y_{3^{(j)}}[i] \mid V_{2^{(j)}}[i], V_{3^{(j)}}[i]\right) \\
& +\sum_{j} \sum_{i=1}^{n} H\left(Y_{2^{(j)}}[i] \mid V_{1^{(2 j+1)}}[i], V_{2^{(j)}}[i]\right) \\
& +\sum_{j} \sum_{i=1}^{n} H\left(Y_{1^{(2 j)}}^{n} \mid V_{1^{(2 j)}}[i], V_{2^{(j)}}[i], V_{3^{(j)}}[i]\right)
\end{aligned}
$$

$\stackrel{(b)}{\leq} \sum_{i=1}^{n} k H\left(Y_{1^{(1)}}[i] \mid V_{3^{(1)}}[i]\right)+k H\left(Y_{3^{(1)}}[i] \mid V_{2^{(1)}}[i], V_{3^{(1)}}[i]\right)$

$+\sum_{i=1}^{n} k H\left(Y_{2^{(1)}}[i] \mid V_{1^{(1)}}[i], V_{2^{(1)}}[i]\right)$

$+\sum_{i=1}^{n} k H\left(Y_{1^{(1)}}[i] \mid V_{1^{(1)}}[i], V_{2^{(1)}}[i], V_{3^{(1)}}[i]\right)$

where step $(a)$ follows from $\left(V_{2^{(j)}}[i], V_{3^{(j+1)}}[i]\right)=$ $h_{1}\left(X_{1^{(2 j+1)}}[i], Y_{1^{(2 j+1)}}[i]\right), \quad\left(V_{1^{(2 j+1)}}[i], V_{3(j-1)}[i]\right)=$ $h_{2}\left(X_{2^{(j)}}[i], Y_{2^{(j)}}[i]\right), \quad\left(V_{1^{(2 j)}}[i], V_{2^{(j)}}[i]\right)=$ $h_{3}\left(X_{3^{(j)}}[i], Y_{3^{(j)}}[i]\right), \quad V_{3^{(j+1)}}[i]=g_{3}\left(X_{3^{(j+1)}}[i]\right)$, $V_{1^{(2 j+1)}}[i]=g_{1}\left(X_{1^{(2 j+1)}}[i]\right)$, and $V_{2^{(j)}}[i]=g_{2}\left(X_{2^{(j)}}[i]\right)$. Step (b) follows, since the coding schemes of $S_{1^{(j)}} \mathrm{s}, S_{2^{(j)}} \mathrm{S}$, and $S_{3^{(j)}} \mathrm{s}$ are the same respectively, $Y_{1^{(j)}}[i] \mathrm{s}, Y_{2^{(j)}}[i] \mathrm{s}$, and $Y_{3^{(j)}}[i]$ s have the same probability distribution respectively.

Proof of part (b) for bound (31).

$$
\begin{aligned}
& H\left(Y_{\Omega_{1}^{c}}^{n}\right)+\sum_{l=2}^{4 k+2} H\left(Y_{\Omega_{l}^{c} \cap \Omega_{l-1}}^{n} \mid W_{\mathcal{S} \backslash \Omega_{l}}, Y_{\Omega_{l-1}^{c}}^{n}\right) \\
& \leq \sum_{j} H\left(Y_{1^{(2 j+1)}}^{n} \mid X_{3^{(j+1)}}^{n}\right)+H\left(Y_{2^{(j)}}^{n} \mid Y_{1^{(2 j+1)}}^{n}, X_{1^{(2 j+1)}}^{n}\right) \\
& +\sum_{j} H\left(Y_{3^{(j)}}^{n} \mid Y_{2^{(j)}}^{n}, X_{2^{(j)}}^{n}, X_{1(2 j+1)}^{n}\right) \\
& +\sum_{j} H\left(Y_{1^{(2 j)}}^{n} \mid Y_{2^{(j)}}^{n}, X_{2^{(j)}}^{n}, X_{3^{(j)}}^{n}\right) \\
& \leq \sum_{j} \sum_{i=1}^{n} H\left(Y_{1(2 j+1)}[i] \mid X_{3(j+1)}[i]\right) \\
& +\sum_{j} \sum_{i=1}^{n} H\left(Y_{2^{(j)}}[i] \mid Y_{1^{(2 j+1)}}[i], X_{1^{(2 j+1)}}[i]\right) \\
& +\sum_{j} \sum_{i=1}^{n} H\left(Y_{3^{(j)}}[i] \mid Y_{2^{(j)}}[i], X_{2^{(j)}}[i], X_{1^{(2 j+1)}}[i]\right) \\
& +\sum_{j} \sum_{i=1}^{n} H\left(Y_{1^{(2 j)}}[i] \mid Y_{2^{(j)}}[i], X_{2^{(j)}}[i], X_{3^{(j)}}[i]\right) \\
& \stackrel{(a)}{\leq} \sum_{j} \sum_{i=1}^{n} H\left(Y_{1^{(2 j+1)}}[i] \mid V_{3^{(j+1)}}[i]\right)+H\left(Y_{2^{(j)}}[i] \mid V_{2^{(j)}}[i]\right) \\
& +\sum_{j} \sum_{i=1}^{n} H\left(Y_{3^{(j)}}[i] \mid V_{1^{(2 j+1)}}[i], V_{2^{(j)}}[i], V_{3^{(j)}}[i]\right) \\
& +\sum_{j} \sum_{i=1}^{n} H\left(Y_{1^{(2 j)}}[i] \mid V_{1^{(2 j)}}[i], V_{2^{(j)}}[i], V_{3^{(j)}}[i]\right) \\
& \stackrel{(b)}{\leq} \sum_{i=1}^{n} k H\left(Y_{1^{(1)}}[i] \mid V_{3^{(1)}}[i]\right)+k H\left(Y_{2^{(1)}}[i] \mid V_{2^{(1)}}[i]\right) \\
& +\sum_{i=1}^{n} k H\left(Y_{3^{(1)}}[i] \mid V_{1^{(1)}}[i], V_{2^{(1)}}[i], V_{3^{(1)}}[i]\right) \\
& +\sum_{i=1}^{n} k H\left(Y_{1^{(1)}}[i] \mid V_{1^{(1)}}[i], V_{2^{(1)}}[i], V_{3^{(1)}}[i]\right)
\end{aligned}
$$

where step $(a)$ follows from $\left(V_{2^{(j)}}[i], V_{3^{(j+1)}}[i]\right)=$ $h_{1}\left(X_{1^{(2 j+1)}}[i], Y_{1^{(2 j+1)}}[i]\right), \quad\left(V_{1^{(2 j)}}[i], V_{3^{(j)}}[i]\right) \quad=$ $h_{2}\left(X_{2^{(j)}}[i], Y_{2^{(j)}}[i]\right), \quad\left(V_{1^{(2 j+1)}}[i], V_{2^{(j)}}[i]\right)=$ $h_{3}\left(X_{3^{(j)}}[i], Y_{3^{(j)}}[i]\right), \quad V_{3^{(j+1)}}[i]=g_{3}\left(X_{3^{(j+1)}}[i]\right)$, 
$V_{1^{(2 j+1)}}[i]=g_{1}\left(X_{1^{(2 j+1)}}[i]\right)$, and $V_{2^{(j)}}[i]=g_{2}\left(X_{2^{(j)}}[i]\right)$. Step (b) follows, since the coding schemes of $S_{1^{(j)}} \mathrm{s}, S_{2^{(j)}} \mathrm{s}$, and $S_{3^{(j)}} \mathrm{s}$ are the same respectively, $Y_{1^{(j)}}[i] \mathrm{s}, Y_{2^{(j)}}[i] \mathrm{s}$, and $Y_{3^{(j)}}[i]$ s have the same probability distribution respectively.

Proof of part (b) for bound (32).

$$
\begin{aligned}
& H\left(Y_{\Omega_{1}^{c}}^{n}\right)+\sum_{l=2}^{4 k+2} H\left(Y_{\Omega_{l}^{c} \cap \Omega_{l-1}}^{n} \mid W_{\mathcal{S} \backslash \Omega_{l}}, Y_{\Omega_{l-1}^{c}}^{n}\right) \\
& \leq \sum_{j} H\left(Y_{3^{(j)}}^{n} \mid Y_{1^{(2 j+3)}}^{n}, X_{1^{(2 j+3)}}^{n}\right)+H\left(Y_{1^{(2 j+1)}}^{n} \mid Y_{3^{(j)}}^{n}, X_{3^{(j)}}^{n}\right) \\
& +\sum_{j} H\left(Y_{2^{(j)}}^{n} \mid Y_{3^{(j)}}^{n}, X_{3^{(j)}}^{n}\right)+H\left(Y_{1^{(2 j)}}^{n} \mid Y_{2^{(j)}}^{n}, X_{2^{(j)}}^{n}, X_{3^{(j)}}^{n}\right) \\
& \leq \sum_{j=1} \sum_{i=1}^{n} H\left(Y_{3^{(j)}}[i] \mid Y_{1^{(2 j+3)}}[i], X_{1(2 j+3)}[i]\right) \\
& +\sum_{j=1} \sum_{i=1}^{n} H\left(Y_{1^{(2 j+1)}}[i] \mid Y_{3^{(j)}}[i], X_{3^{(j)}}[i]\right) \\
& +\sum_{j=1} \sum_{i=1}^{n} H\left(Y_{2^{(j)}}[i] \mid Y_{3^{(j)}}[i], X_{3^{(j)}}[i]\right) \\
& +\sum_{j=1} \sum_{i=1}^{n} H\left(Y_{1^{(2 j)}}[i] \mid Y_{2^{(j)}}^{n}, X_{2^{(j)}}^{n}, X_{3^{(j)}}^{n}\right) \\
& \stackrel{(a)}{\leq} \sum_{j=1} \sum_{i=1}^{n} H\left(Y_{3^{(j)}}[i] \mid V_{3^{(j)}}[i]\right)+H\left(Y_{1^{(2 j+1)}}[i] \mid V_{1^{(2 j+1)}}[i], V_{2^{(j)}}[i]\right) \\
& +\sum_{j=1} \sum_{i=1}^{n} H\left(Y_{2^{(j)}}[i] \mid V_{2^{(j)}}[i], V_{3^{(j)}}[i]\right) \\
& +\sum_{j=1} \sum_{i=1}^{n} H\left(Y_{1^{(2 j)}}[i] \mid V_{1^{(2 j)}}[i], V_{2^{(j)}}[i], V_{3^{(j)}}[i]\right) \\
& \stackrel{(b)}{\leq} \sum_{i=1}^{n} k H\left(Y_{3^{(1)}}[i] \mid V_{3^{(1)}}[i]\right)+k H\left(Y_{1^{(1)}}[i] \mid V_{1^{(1)}}[i], V_{2^{(1)}}[i]\right) \\
& +\sum_{i=1}^{n} k H\left(Y_{2^{(1)}}[i] \mid V_{2^{(1)}}[i], V_{3^{(1)}}[i]\right) \\
& +\sum_{i=1}^{n} k H\left(Y_{1^{(1)}}[i] \mid V_{1^{(1)}}[i], V_{2^{(1)}}[i], V_{3^{(1)}}[i]\right)
\end{aligned}
$$

where step $(a)$ follows from $\left(V_{2^{(j+1)}}[i], V_{3^{(j)}}[i]\right)=$ $h_{1}\left(X_{1^{(2 j+3)}}[i], Y_{1(2 j+3)}[i]\right), \quad\left(V_{2^{(j)}}[i], V_{3^{(j-1)}}[i]\right)=$ $h_{1}\left(X_{1^{(2 j+1)}}[i], Y_{1^{(2 j+1)}}[i]\right), \quad\left(V_{1^{(2 j)}}[i], V_{3^{(j)}}[i]\right)=$ $h_{2}\left(X_{2^{(j)}}[i], Y_{2^{(j)}}[i]\right), \quad\left(V_{1^{(2 j+1)}}[i], V_{2^{(j)}}[i]\right)=$ $h_{3}\left(X_{3^{(j)}}[i], Y_{3^{(j)}}[i]\right), \quad V_{3^{(j+1)}}[i]=g_{3}\left(X_{3^{(j+1)}}[i]\right)$, $V_{1^{(2 j+1)}}[i]=g_{1}\left(X_{1^{(2 j+1)}}[i]\right)$, and $V_{2^{(j)}}[i]=g_{2}\left(X_{2^{(j)}}[i]\right)$. Step (b) follows, since the coding schemes of $S_{1^{(j)}} \mathrm{s}, S_{2^{(j)}} \mathrm{s}$, and $S_{3^{(j)}} \mathrm{s}$ are the same respectively, $Y_{1^{(j)}}[i] \mathrm{s}, Y_{2^{(j)}}[i] \mathrm{s}$, and $Y_{3^{(j)}}[i] \mathrm{s}$ have the same probability distribution respectively.

Proof of part (b) for bound (33).

$$
\begin{aligned}
& H\left(Y_{\Omega_{1}^{c}}^{n}\right)+\sum_{l=2}^{4} H\left(Y_{\Omega_{l}^{c} \cap \Omega_{l-1}}^{n} \mid W_{\mathcal{S} \backslash \Omega_{l}}, Y_{\Omega_{l-1}^{c}}^{n}\right) \\
& \leq H\left(Y_{2^{(1)}}^{n}\right)+H\left(Y_{1^{(2)}}^{n} \mid Y_{2^{(1)}}^{n}, X_{2^{(1)}}^{n}\right)+H\left(Y_{3^{(1)}}^{n} \mid Y_{2^{(1)}}^{n}, X_{2^{(1)}}^{n}\right) \\
& +H\left(Y_{1^{(1)}}^{n} \mid Y_{3^{1}}^{n}, X_{3^{1}}^{n}, X_{2^{1}}^{n}\right)
\end{aligned}
$$

$$
\begin{aligned}
& \stackrel{(a)}{\leq} \sum_{i=1}^{n} H\left(Y_{2^{(1)}}[i]\right)+H\left(Y_{1^{(2)}}[i] \mid V_{1^{(2)}}[i], V_{2^{(1)}}[i], V_{3^{(1)}}[i]\right) \\
& +\sum_{i=1}^{n} H\left(Y_{3^{(1)}}[i] \mid V_{2^{(1)}}[i], V_{3^{(1)}}[i]\right) \\
& +\sum_{i=1}^{n}+H\left(Y_{1^{(1)}}[i] \mid V_{1^{(1)}}[i], V_{2^{(1)}}[i], V_{3^{(1)}}[i]\right) \\
& \stackrel{(b)}{\leq} \sum_{i=1}^{n} H\left(Y_{2^{(1)}}[i]\right)+H\left(Y_{1^{(1)}}[i] \mid V_{1^{(1)}}[i], V_{2^{(1)}}[i], V_{3^{(1)}}[i]\right) \\
& +\sum_{i=1}^{n} H\left(Y_{3^{(1)}}[i] \mid V_{2^{(1)}}[i], V_{3^{(1)}}[i]\right) \\
& +\sum_{i=1}^{n} H\left(Y_{1^{(1)}}[i] \mid V_{1^{(1)}}[i], V_{2^{(1)}}[i], V_{3^{(1)}}[i]\right)
\end{aligned}
$$

where $(a)$ follows from $V_{1^{(2)}}[i]=g_{1}\left(X_{1^{(2)}}[i]\right)$, $\left(V_{2^{(1)}}[i], V_{3^{(1)}}[i]\right)=h_{1}\left(X_{1^{(2)}}[i], Y_{1^{(2)}}[i]\right)$, $V_{3^{(1)}}[i]=g_{3}\left(X_{3^{(1)}}[i]\right), \quad V_{2^{(1)}}[i]=g_{2}\left(X_{2^{(1)}}[i]\right)$, $\left(V_{1^{(1)}}[i], V_{2^{(1)}}[i]\right)=h_{3}\left(X_{3^{(1)}}[i], Y_{3^{(1)}}[i]\right), \quad$ and $\left(V_{1^{(1)}}[i], V_{3^{(1)}}[i]\right)=h_{2}\left(X_{2^{(1)}}[i], Y_{2^{(1)}}[i]\right)$. Step $(b)$ follows, since the coding schemes of $S_{1^{(j)}}$ and $S_{2^{(j)}}$ are the same respectively, $Y_{1^{(j)}}[i] \mathrm{s}$ and $Y_{2^{(j)}}[i] \mathrm{s}$ have the same probability distribution respectively.

Proof of part (b) for bound (34).

$$
\begin{aligned}
& H\left(Y_{\Omega_{1}^{c}}^{n}\right)+\sum_{l=2}^{4 k+2} H\left(Y_{\Omega_{l}^{c}}^{n} \cap \Omega_{l-1} \mid W_{\mathcal{S} \backslash \Omega_{l}}, Y_{\Omega_{l-1}^{c}}^{n}\right) \\
& \leq \sum_{j} H\left(Y_{3^{(j)}}^{n} \mid Y_{2^{(j+1)}}^{n}, X_{2^{(j+1)}}^{n}\right)+H\left(Y_{2^{(j)}}^{n} \mid Y_{3^{(j)}}^{n}, X_{3^{(j)}}^{n}\right) \\
& +\sum_{j} H\left(Y_{1^{(2 j+1)}}^{n} \mid Y_{3^{(j)}}^{n}, X_{3^{(j)}}^{n}, X_{2^{(j)}}^{n}\right) \\
& +\sum_{j} H\left(Y_{1^{(2 j)}}^{n} \mid Y_{2^{(j)}}^{n}, X_{2^{(j)}}^{n}, X_{3^{(j)}}^{n}\right) \\
& \stackrel{(a)}{\leq} \sum_{j} \sum_{i=1}^{n} H\left(Y_{3^{(j)}}[i] \mid V_{3^{(j)}}[i]\right)+H\left(Y_{2^{(j)}}[i] \mid V_{2^{(j)}}[i]\right) \\
& +\sum_{j} \sum_{i=1}^{n} H\left(Y_{1^{(2 j+1)}}[i] \mid V_{1^{(2 j+1)}}[i], V_{2^{(j)}}[i], V_{3^{(j)}}[i]\right) \\
& +\sum_{j} \sum_{i=1}^{n} H\left(Y_{1^{(2 j)}}[i] \mid V_{1^{(2 j)}}[i], V_{2^{(j)}}[i], V_{3^{(j)}}[i]\right) \\
& \stackrel{(b)}{\leq} \sum_{i=1}^{n} k H\left(Y_{3^{(1)}}[i] \mid V_{3^{(1)}}[i]\right)+k H\left(Y_{2^{(1)}}[i] \mid V_{2^{(1)}}[i]\right) \\
& +\sum_{i=1}^{n} k H\left(Y_{1^{(1)}}[i] \mid V_{1^{(1)}}[i], V_{2^{(1)}}[i], V_{3^{(1)}}[i]\right) \\
& +\sum_{i=1}^{n} k H\left(Y_{1^{(1)}}[i] \mid V_{1^{(1)}}[i], V_{2^{(1)}}[i], V_{3^{(1)}}[i]\right)
\end{aligned}
$$

where step $(a)$ follows from $\left(V_{1^{(2 j)}}[i], V_{3^{(j-1)}}[i]\right)=$ $h_{2}\left(X_{2^{(j)}}[i], Y_{2^{(j)}}[i]\right), \quad\left(V_{1^{(2 j)}}[i], V_{3^{(j)}}[i]\right)=$ $h_{2}\left(X_{2^{(j+1)}}[i], Y_{2^{(j+1)}}[i]\right), \quad\left(V_{1^{(2 j+1)}}[i], V_{2^{(j)}}[i]\right)=$ $h_{3}\left(X_{3^{(j)}}[i], Y_{3^{(j)}}[i]\right), \quad V_{3^{(j)}}[i]=g_{3}\left(X_{3^{(j)}}[i]\right), \quad$ and $V_{2^{(j)}}[i]=g_{2}\left(X_{2^{(j)}}[i]\right)$. Step (b) follows, since the coding schemes of $S_{1^{(j)}} \mathrm{S}, S_{2^{(j)}} \mathrm{S}$, and $S_{3^{(j)}} \mathrm{S}$ are the same respectively, 
$Y_{1^{(j)}}[i] \mathrm{s}, Y_{2^{(j)}}[i] \mathrm{s}$, and $Y_{3^{(j)}}[i] \mathrm{s}$ have the same probability distribution respectively.

\section{Proof of part (b) for bound (35).}

$$
\begin{aligned}
& H\left(Y_{\Omega_{1}^{c}}^{n}\right)+\sum_{l=2}^{5} H\left(Y_{\Omega_{l}^{c} \cap \Omega_{l-1}}^{n} \mid W_{\mathcal{S} \backslash \Omega_{l}}, Y_{\Omega_{l-1}^{c}}^{n}\right) \\
& \leq H\left(Y_{1^{(3)}}^{n}\right)+H\left(Y_{3^{(1)}}^{n} \mid Y_{1^{(3)}}^{n}, X_{1^{(3)}}^{n}\right)+H\left(Y_{1^{(2)}}^{n} \mid Y_{3^{(1)}}^{n}, X_{3^{(1)}}^{n}\right) \\
& +H\left(Y_{2^{(1)}}^{n} \mid Y_{3^{1}}^{n}, X_{3^{1}}^{n}\right)+H\left(Y_{1^{(1)}}^{n} \mid Y_{2^{(1)}}^{n}, X_{2^{(1)}}^{n}, X_{3^{(1)}}^{n}\right) \\
& \stackrel{(a)}{\leq} \sum_{i=1}^{n} H\left(Y_{1^{(3)}}[i]\right)+H\left(Y_{3^{(1)}}[i] \mid V_{2^{(1)}}[i], V_{3^{(1)}}[i]\right) \\
& +\sum_{i=1}^{n} H\left(Y_{1^{(2)}}[i] \mid V_{1^{(2)}}[i], V_{2^{(1)}}[i], V_{3^{(1)}}[i]\right) \\
& +\sum_{i=1}^{n} H\left(Y_{2^{(1)}}[i] \mid V_{2^{(1)}}[i], V_{3^{(1)}}[i]\right) \\
& +\sum_{i=1}^{n} H\left(Y_{1^{(1)}}[i] \mid V_{1^{(1)}}[i], V_{2^{(1)}}[i], V_{3^{(1)}}[i]\right) \\
& \quad \sum_{i=1}^{(b)} H\left(Y_{1^{(1)}}[i]\right)+H\left(Y_{3^{(1)}}[i] \mid V_{2^{(1)}}[i], V_{3^{(1)}}[i]\right) \\
& +\sum_{i=1}^{n} H\left(Y_{1^{(1)}}[i] \mid V_{1^{(1)}}[i], V_{2^{(1)}}[i], V_{3^{(1)}}[i]\right) \\
& +\sum_{i=1}^{n} H\left(Y_{2^{(1)}}[i] \mid V_{2^{(1)}}[i], V_{3^{(1)}}[i]\right) \\
& +\sum_{i=1}^{n} H\left(Y_{1^{(1)}}[i] \mid V_{1^{(1)}}[i], V_{2^{(1)}}[i], V_{3^{(1)}}[i]\right)
\end{aligned}
$$

where $(a)$ follows from $V_{1^{(2)}}[i]=g_{1}\left(X_{1^{(2)}}[i]\right)$, $\left(V_{2^{(1)}}[i], V_{3^{(1)}}[i]\right)=h_{1}\left(X_{1^{(3)}}[i], Y_{1^{(3)}}[i]\right)$, $V_{3^{(1)}}[i]=g_{3}\left(X_{3^{(1)}}[i]\right), \quad V_{2^{(1)}}[i]=g_{2}\left(X_{2^{(1)}}[i]\right)$, $\left(V_{1^{(2)}}[i], V_{2^{(1)}}[i]\right)=h_{3}\left(X_{3^{(1)}}[i], Y_{3^{(1)}}[i]\right), \quad$ and $\left(V_{1^{(1)}}[i], V_{3^{(1)}}[i]\right)=h_{2}\left(X_{2^{(1)}}[i], Y_{2^{(1)}}[i]\right)$. Step $(b)$ follows, since the coding schemes of $S_{1(j)}$ and $S_{2^{(j)}}$ are the same respectively, $Y_{1^{(j)}}[i] \mathrm{s}$ and $Y_{2^{(j)}}[i] \mathrm{s}$ have the same probability distribution respectively.

\section{Proof of part (b) for bound (36).}

$$
\begin{aligned}
& H\left(Y_{\Omega_{1}^{c}}^{n}\right)+\sum_{l=2}^{5 k+2} H\left(Y_{\Omega_{l}^{c} \cap \Omega_{l-1}}^{n} \mid W_{\mathcal{S} \backslash \Omega_{l}}, Y_{\Omega_{l-1}^{c}}^{n}\right) \\
& \leq \sum_{j} H\left(Y_{1^{(3 j+2)}}^{n} \mid X_{2^{(j+1)}}^{n}\right)+H\left(Y_{3^{(j)}}^{n} \mid Y_{1^{(3 j+2)}}^{n}, X_{1^{(3 j+2)}}^{n}\right) \\
& +\sum_{j} H\left(Y_{2^{(j)}}^{n} \mid Y_{3^{(j)}}^{n}, X_{3^{(j)}}^{n}\right)+H\left(Y_{1^{(3 j+1)}}^{n} \mid Y_{3^{(j)}}^{n}, X_{3^{(j)}}^{n}, X_{2^{(j)}}^{n}\right) \\
& +\sum_{j} H\left(Y_{1^{(3 j)}}^{n} \mid Y_{2^{(j)}}^{n}, X_{2^{(j)}}^{n}, X_{3^{(j)}}^{n}\right) \\
& \stackrel{(a)}{\leq} \sum_{j} \sum_{i=1}^{n} H\left(Y_{1^{(3 j+2)}}[i] \mid V_{2^{(j+1)}}[i]\right)+H\left(Y_{3^{(j)}}[i] \mid V_{3^{(j)}}[i]\right) \\
& +\sum_{j} \sum_{i=1}^{n} H\left(Y_{2^{(j)}}[i] \mid V_{2^{(j)}}[i], V_{3^{(j)}}[i]\right)
\end{aligned}
$$

$+\sum_{j} \sum_{i=1}^{n} H\left(Y_{1^{(3 j+1)}}[i] \mid V_{1^{(3 j+1)}}[i], V_{2^{(j)}}[i], V_{3^{(j)}}[i]\right)$

$+\sum_{j} \sum_{i=1}^{n} H\left(Y_{1^{(3 j)}}[i] \mid V_{1^{(3 j)}}[i], V_{2^{(j)}}[i], V_{3^{(j)}}[i]\right)$

$\stackrel{(b)}{\leq} \sum_{i=1}^{n} k H\left(Y_{1^{(1)}}[i] \mid V_{2^{(1)}}[i]\right)+2 k H\left(Y_{1^{(1)}}[i] \mid V_{1^{(1)}}[i], V_{2^{(1)}}[i], V_{3^{(1)}}[i]\right)$

$+\sum_{i=1}^{n} k H\left(Y_{2^{(1)}}[i] \mid V_{2^{(1)}}[i], V_{3^{(1)}}[i]\right)+k H\left(Y_{3^{(1)}}[i] \mid V_{3^{(1)}}[i]\right)$

where step $(a)$ follows from $\left(V_{1^{(3 j)}}[i], V_{3^{(j)}}[i]\right)=$ $h_{2}\left(X_{2^{(j)}}[i], Y_{2^{(j)}}[i]\right), \quad\left(V_{2^{(j)}}[i], V_{3^{(j)}}[i]\right)=$ $h_{1}\left(X_{1^{(3 j+1)}}[i], Y_{1^{(3 j+1)}}[i]\right), \quad\left(V_{1^{(3 j+1)}}[i], V_{2^{(j)}}[i]\right)=$ $h_{3}\left(X_{3^{(j)}}[i], Y_{3^{(j)}}[i]\right), \quad V_{3^{(j)}}[i]=g_{3}\left(X_{3^{(j)}}[i]\right), \quad$ and $V_{2^{(j)}}[i]=g_{2}\left(X_{2^{(j)}}[i]\right)$. Step (b) follows, since the coding schemes of $S_{1^{(j)}} \mathrm{s}, S_{2^{(j)}} \mathrm{s}$, and $S_{3^{(j)}} \mathrm{s}$ are the same respectively, $Y_{1^{(j)}}[i] \mathrm{s}, Y_{2^{(j)}}[i] \mathrm{s}$, and $Y_{3^{(j)}}[i] \mathrm{s}$ have the same probability distribution respectively.

Proof of part (b) for bound (37).

$$
\begin{aligned}
& H\left(Y_{\Omega_{1}^{c}}^{n}\right)+\sum_{l=2}^{5} H\left(Y_{\Omega_{l}^{c}}^{n} \cap \Omega_{l-1} \mid W_{\mathcal{S} \backslash \Omega_{l}}, Y_{\Omega_{l-1}^{c}}^{n}\right) \\
& =H\left(Y_{1^{(2)}}^{n}\right)+H\left(Y_{3^{(1)}}^{n} \mid W_{1^{(2)}}, Y_{1^{(2)}}^{n}\right) \\
& +H\left(Y_{1^{(1)}}^{n} \mid W_{\left[3^{(1)}, 1^{(2)}\right]}, Y_{\left[3^{(1)}, 1^{(2)}\right]}^{n}\right) \\
& +H\left(Y_{2^{(2)}}^{n} \mid W_{\left[1^{(1)}, 3^{(1)}, 1^{(2)}\right]}, Y_{\left[1^{(1)}, 3^{(1)}, 1^{(2)}\right]}^{n}\right) \\
& +H\left(Y_{2^{(1)}}^{n} \mid W_{\left[2^{(2)}, 1^{(1)}, 3^{(1)}, 1^{(2)}\right]}, Y_{\left[2^{(2)}, 1^{(1)}, 3^{(1)}, 1^{(2)}\right]}^{n}\right) \\
& =H\left(Y_{1^{(2)}}^{n}\right)+H\left(Y_{3^{(1)}}^{n} \mid W_{1^{(2)}}, Y_{1^{(2)}}^{n}, X_{1^{(2)}}^{n}\right) \\
& +H\left(Y_{1^{(1)}}^{n} \mid W_{\left[3^{(1)}, 1^{(2)}\right]}, Y_{\left[3^{(1)}, 1^{(2)}\right]}^{n}, X_{\left[3^{(1)}, 1^{(2)}\right]}^{n}\right) \\
& +H\left(Y_{2^{(2)}}^{n} \mid W_{\left[1^{(1)}, 3^{(1)}, 1^{(2)}\right]}, Y_{\left[1^{(1)}, 3^{(1)}, 1^{(2)}\right]}^{n}, X_{\left[1^{(1)}, 3^{(1)}, 1^{(2)}\right]}^{n}\right) \\
& +H\left(Y_{2^{(1)}}^{n} \mid W_{\left[2^{(2)}, 1^{(1)}, 3^{(1)}, 1^{(2)}\right]}, Y_{\left[2^{(2)}, 1^{(1)}, 3^{(1)}, 1^{(2)}\right]}^{n}, X_{\left[2^{(2)}, 1^{(1)}, 3^{(1)}, 1^{(2)}\right]}^{n}\right) \\
& \leq H\left(Y_{1(2)}^{n}\right)+H\left(Y_{3^{(1)}}^{n} \mid Y_{1(2)}^{n}, X_{1(2)}^{n}\right) \\
& +H\left(Y_{1^{(1)}}^{n} \mid Y_{\left[3^{(1)}, 1^{(2)}\right]}^{n}, X_{\left[3^{(1)}, 1^{(2)}\right]}^{n}\right) \\
& +H\left(Y_{2^{(2)}}^{n} \mid Y_{\left[1^{(1)}, 3^{(1)}, 1^{(2)}\right]}^{n}, X_{\left[1^{(1)}, 3^{(1)}, 1^{(2)}\right]}^{n}\right) \\
& +H\left(Y_{2^{(1)}}^{n} \mid Y_{\left[2^{(2)}, 1^{(1)}, 3^{(1)}, 1^{(2)}\right]}^{n}, X_{\left[2^{(2)}, 1^{(1)}, 3^{(1)}, 1^{(2)}\right]}^{n}\right) \\
& =\sum_{i} H\left(Y_{1(2)}[i] \mid Y_{1(2)}^{i-1}\right) \\
& +\sum_{i} H\left(Y_{3^{(1)}}[i] \mid Y_{3(1)}^{i-1}, Y_{1(2)}^{n}, X_{1(2)}^{n}\right) \\
& +\sum_{i} H\left(Y_{1^{(1)}}[i] \mid Y_{1^{(1)}}^{i-1}, Y_{\left[3^{(1)}, 1^{(2)}\right]}^{n}, X_{\left[3^{(1)}, 1^{(2)}\right]}^{n}\right) \\
& +\sum_{i} H\left(Y_{2^{(2)}}[i] \mid Y_{2^{(2)}}^{i-1}, Y_{\left[1^{(1)}, 3^{(1)}, 1^{(2)}\right]}^{n}, X_{\left[1^{(1)}, 3^{(1)}, 1^{(2)}\right]}^{n}\right) \\
& +\sum_{i} H\left(Y_{2^{(1)}}[i] \mid Y_{2^{(1)}}^{i-1}, Y_{\left[2^{(2)}, 1^{(1)}, 3^{(1)}, 1^{(2)}\right]}^{n}, X_{\left[2^{(2)}, 1^{(1)}, 3^{(1)}, 1^{(2)}\right]}^{n}\right) \\
& \stackrel{(a)}{\leq} \sum_{i} H\left(Y_{1^{(2)}}[i]\right)+\sum_{i} H\left(Y_{3^{(1)}} \mid V_{3^{(1)}}[i], V_{2^{(2)}}[i]\right) \\
& +\sum_{i} H\left(Y_{1^{(1)}}[i] \mid V_{1^{(1)}}[i], V_{3^{(1)}}[i]\right) \\
& +\sum_{i} H\left(Y_{2^{(2)}}[i] \mid V_{2^{(2)}}[i], V_{1^{(1)}}[i], V_{3^{(1)}}[i]\right) \\
& +\sum_{i} H\left(Y_{2^{(1)}}[i] \mid V_{2^{(1)}}[i], V_{1^{(1)}}[i], V_{3^{(1)}}[i]\right)
\end{aligned}
$$




$$
\begin{aligned}
& \left.\stackrel{(b)}{\leq} \sum_{i} Y_{1^{(1)}}[i]\right)+\sum_{i} H\left(Y_{3^{(1)}} \mid V_{3^{(1)}}[i], V_{2^{(1)}}[i]\right) \\
& +\sum_{i} H\left(Y_{1^{(1)}}[i] \mid V_{1^{(1)}}[i], V_{3^{(1)}}[i]\right) \\
& +2 \sum_{i} H\left(Y_{2^{(1)}}[i] \mid V_{2^{(1)}}[i], V_{1^{(1)}}[i], V_{3^{(1)}}[i]\right)
\end{aligned}
$$

where step $(a)$ follows from $\left(V_{2^{(2)}}[i], V_{3^{(1)}}[i]\right)=$ $h_{1}\left(X_{1^{(2)}}[i], Y_{1^{(2)}}[i]\right), \quad\left(V_{1^{(1)}}[i], V_{2^{(2)}}[i]\right) \quad=$ $h_{3}\left(X_{3^{(1)}}[i], Y_{3^{(1)}}[i]\right), \quad V_{3^{(1)}}[i]=g_{3}\left(X_{3^{(1)}}[i]\right), \quad V_{1^{(1)}}[i]=$ $g_{1}\left(X_{1^{(1)}}[i]\right)$, and $\left(V_{2^{(1)}}[i], V_{3^{(1)}}[i]\right)=h_{1}\left(X_{1^{(1)}}[i], Y_{1^{(1)}}[i]\right)$ . Step $(b)$ follows, since the coding schemes of $S_{1^{(j)} \mathrm{S}}$ and $S_{2^{(j)}} \mathrm{S}$ are the same respectively, $Y_{1^{(j)}}[i] \mathrm{s}$ and $Y_{2^{(j)}}[i] \mathrm{s}$ have the same probability distribution respectively. Note that $V_{2^{(2)}}[i]$ and $V_{2^{(1)}}[i]$ also have the same probability distribution.

Proof of part (b) for bound (38).

$$
\begin{aligned}
& H\left(Y_{\Omega_{1}^{c}}^{n}\right)+\sum_{l=2}^{5} H\left(Y_{\Omega_{l}^{c} \cap \Omega_{l-1}}^{n} \mid W_{\mathcal{S} \backslash \Omega_{l}}, Y_{\Omega_{l-1}^{c}}^{n}\right) \\
& \leq H\left(Y_{1^{(2)}}^{n}\right)+H\left(Y_{2^{(2)}}^{n} \mid Y_{1^{(2)}}^{n}, X_{1^{(2)}}^{n}\right)+H\left(Y_{3^{(1)}}^{n} \mid Y_{1^{(2)}}^{n}, X_{1^{(2)}}^{n}\right) \\
& +H\left(Y_{2^{(1)}}^{n} \mid Y_{3^{1}}^{n}, X_{3^{1}}^{n}\right)+H\left(Y_{1^{(1)}}^{n} \mid Y_{2^{(1)}}^{n}, X_{2^{(1)}}^{n}, X_{3^{(1)}}^{n}\right) \\
& \quad \stackrel{(a)}{\leq} \sum_{i=1}^{n} H\left(Y_{1^{(2)}}[i]\right)+H\left(Y_{2^{(2)}}[i] \mid V_{2^{(2)}}[i], V_{3^{(1)}}[i]\right) \\
& +\sum_{i=1}^{n} H\left(Y_{3^{(1)}}[i] \mid V_{1^{(2)}}[i] V_{3^{(1)}}[i]\right)+H\left(Y_{2^{(1)}}[i] \mid V_{2^{(1)}}[i] V_{3^{(1)}}[i]\right) \\
& +\sum_{i=1}^{n} H\left(Y_{1^{(1)}}[i] \mid V_{1^{(1)}}[i], V_{2^{(1)}}[i], V_{3^{(1)}}[i]\right) \\
& \stackrel{(b)}{\leq} \sum_{i=1}^{n} H\left(Y_{1^{(1)}}[i]\right)+H\left(Y_{2^{(1)}}[i] \mid V_{1^{(1)}}[i], V_{2^{(1)}}[i], V_{3^{(1)}}[i]\right) \\
& +\sum_{i=1}^{n} H\left(Y_{3^{(1)}}[i] \mid V_{1^{(1)}}[i], V_{3^{(1)}}[i]\right)+H\left(Y_{2^{(1)}}[i] \mid V_{2^{(1)}}[i], V_{3^{(1)}}[i]\right) \\
& +\sum_{i=1}^{n} H\left(Y_{1^{(1)}}[i] \mid V_{1^{(1)}}[i], V_{2^{(1)}}[i], V_{3^{(1)}}[i]\right)
\end{aligned}
$$

where $(a)$ follows from $\left(V_{1^{(1)}}[i], V_{3^{(1)}}[i]\right)=$ $h_{2}\left(X_{2^{(1)}}[i], Y_{2^{(1)}}[i]\right), \quad\left(V_{1^{(2)}}[i], V_{3^{(1)}}[i]\right) \quad=$ $h_{2}\left(X_{2^{(2)}}[i], Y_{2^{(2)}}[i]\right), \quad V_{3^{(1)}}[i]=g_{3}\left(X_{3^{(1)}}[i]\right), \quad V_{2^{(1)}}[i]=$ $g_{2}\left(X_{2^{(1)}}[i]\right), V_{2^{(2)}}[i]=g_{2}\left(X_{2^{(2)}}[i]\right), V_{1^{(2)}}[i]=g_{2}\left(X_{1^{(2)}}[i]\right)$, $\left(V_{2^{(2)}}[i], V_{3^{(1)}}[i]\right) \quad=\quad h_{1}\left(X_{1^{(2)}}[i], Y_{1^{(2)}}[i]\right), \quad$ and $\left(V_{1^{(2)}}[i], V_{2^{(2)}}[i]\right)=h_{3}\left(X_{3^{(1)}}[i], Y_{3^{(1)}}[i]\right)$. Step $(b)$ follows, since the coding schemes of $S_{1^{(j)}}$ and $S_{2^{(j)}}$ are the same respectively, $Y_{1^{(j)}}[i] \mathrm{s}$ and $Y_{2^{(j)}}[i] \mathrm{s}$ have the same probability distribution respectively.

Proof of part (b) for bound (39).

$$
\begin{aligned}
& H\left(Y_{\Omega_{1}^{c}}^{n}\right)+\sum_{l=2}^{5} H\left(Y_{\Omega_{l}^{c}}^{n} \cap \Omega_{l-1} \mid W_{\mathcal{S} \backslash \Omega_{l}}, Y_{\Omega_{l-1}^{c}}^{n}\right) \\
& \leq H\left(Y_{1^{(2)}}^{n}\right)+H\left(Y_{3^{(1)}}^{n} \mid Y_{1^{(2)}}^{n}, X_{1^{(2)}}^{n}\right) \\
& +H\left(Y_{2^{(2)}}^{n} \mid Y_{1^{(2)}}^{n}, X_{1^{(2)}}^{n}, Y_{3^{(1)}}^{n}, X_{3^{(1)}}^{n}\right)+H\left(Y_{1^{(1)}}^{n} \mid Y_{3^{1}}^{n}, X_{3^{1}}^{n}\right) \\
& +H\left(Y_{2^{(1)}}^{n} \mid Y_{1^{(1)}}^{n}, X_{1^{(1)}}^{n}, X_{3^{(1)}}^{n}\right) \\
& \stackrel{(a)}{\leq} \sum_{i=1}^{n} H\left(Y_{1^{(2)}}[i]\right)+H\left(Y_{3^{(1)}}[i] \mid V_{3^{(1)}}[i]\right)
\end{aligned}
$$

$$
\begin{aligned}
& +\sum_{i=1}^{n} H\left(Y_{2^{(2)}}[i] \mid V_{1^{(1)}}[i] V_{2^{(2)}}[i] V_{3^{(1)}}[i]\right) \\
& +\sum_{i=1}^{n} H\left(Y_{1^{(1)}}[i] \mid V_{1^{(1)}}[i] V_{2^{(1)}}[i] V_{3^{(1)}}[i]\right) \\
& +\sum_{i=1}^{n} H\left(Y_{2^{(1)}}[i] \mid V_{1^{(1)}}[i] V_{2^{(1)}}[i] V_{3^{(1)}}[i]\right) \\
& \stackrel{(b)}{\leq} \sum_{i=1}^{n} H\left(Y_{1^{(1)}}[i]\right)+H\left(Y_{3^{(1)}}[i] \mid V_{3^{(1)}}[i]\right) \\
& +\sum_{i=1}^{n} H\left(Y_{2^{(1)}}[i] \mid V_{1^{(1)}}[i] V_{2^{(1)}}[i] V_{3^{(1)}}[i]\right) \\
& +\sum_{i=1}^{n} H\left(Y_{1^{(1)}}[i] \mid V_{1^{(1)}}[i] V_{2^{(1)}}[i] V_{3^{(1)}}[i]\right) \\
& +\sum_{i=1}^{n} H\left(Y_{2^{(1)}}[i] \mid V_{1^{(1)}}[i] V_{2^{(1)}}[i] V_{3^{(1)}}[i]\right)
\end{aligned}
$$

where $(a)$ follows from $\left(V_{2^{(2)}}[i], V_{3^{(1)}}[i]\right)=$ $h_{1}\left(X_{1^{(2)}}[i], Y_{1^{(2)}}[i]\right), \quad V_{3^{(1)}}[i]=g_{3}\left(X_{3^{(1)}}[i]\right), \quad V_{1^{(1)}}[i]=$ $g_{1}\left(X_{1^{(1)}}[i]\right), \quad\left(V_{1^{(1)}}[i], V_{2^{(1)}}[i]\right)=h_{3}\left(X_{3^{(1)}}[i], Y_{3^{(1)}}[i]\right)$, $\left(V_{2^{(1)}}[i], V_{3^{(1)}}[i]\right)=h_{1}\left(X_{1^{(1)}}[i], Y_{1^{(1)}}[i]\right), \quad=\quad$ and $\left(V_{1^{(1)}}[i], V_{3^{(1)}}[i]\right)=h_{2}\left(X_{2^{(2)}}[i], Y_{2^{(2)}}[i]\right)$. Step $(b)$ follows, since the coding schemes of $S_{1^{(j)}}$ and $S_{2^{(j)}}$ are the same respectively, $Y_{1^{(j)}}[i] \mathrm{s}$ and $Y_{2^{(j)}}[i] \mathrm{s}$ have the same probability distribution respectively.

Proof of part (b) for bound 40.

$$
\begin{aligned}
& H\left(Y_{\Omega_{1}^{c}}^{n}\right)+\sum_{l=2}^{5 k+2} H\left(Y_{\Omega_{l}^{c} \cap \Omega_{l-1}}^{n} \mid W_{\mathcal{S} \backslash \Omega_{l}}, Y_{\Omega_{l-1}^{c}}^{n}\right) \\
& \leq \sum_{j} H\left(Y_{1^{(2 j+1)}}^{n} \mid Y_{3^{(j+1)}}^{n} X_{3^{(j+1)}}^{n}\right) \\
& +\sum_{j} H\left(Y_{2^{(2 j+1)}}^{n} \mid Y_{1^{(2 j+1)}}^{n} X_{1^{(2 j+1)}}^{n}\right) \\
& +\sum_{j} H\left(Y_{3^{(j)}}^{n} \mid Y_{1(2 j+1)}^{n} X_{1(2 j+1)}^{n}\right) \\
& +\sum_{j} H\left(Y_{1^{(2 j)}}^{n} \mid Y_{2^{(2 j+1)}}^{n} X_{2^{(2 j+1)}}^{n} X_{3^{(j)}}^{n}\right) \\
& +\sum_{j} H\left(Y_{2^{(2 j)}}^{n} \mid Y_{3^{(j)}}^{n} X_{3^{(j)}}^{n} X_{1(2 j)}^{n}\right) \\
& \stackrel{(a)}{\leq} \sum_{j} \sum_{i=1}^{n} H\left(Y_{1^{(2 j+1)}}[i] \mid V_{1^{(2 j+1)}}[i]\right) \\
& +\sum_{j} \sum_{i=1}^{n} H\left(Y_{2^{(2 j+1)}}[i] \mid V_{2^{(2 j+1)}}[i] V_{3^{(j)}}[i]\right) \\
& +\sum_{j} \sum_{i=1}^{n} H\left(Y_{3^{(j)}}[i] \mid V_{3^{(j)}}[i]\right) \\
& +\sum_{j} \sum_{i=1}^{n} H\left(Y_{1^{(2 j)}}[i] \mid V_{1^{(2 j)}}[i], V_{2^{(2 j+1)}}[i], V_{3^{(j)}}[i]\right) \\
& +\sum_{j} \sum_{i=1}^{n} H\left(Y_{2^{(2 j)}}[i] \mid V_{1^{(2 j)}}[i], V_{2^{(2 j)}}[i], V_{3^{(j)}}[i]\right)
\end{aligned}
$$




$$
\begin{aligned}
& \stackrel{(b)}{\leq} \sum_{i=1}^{n} k H\left(Y_{1^{(1)}}[i] \mid V_{1^{(1)}}[i]\right)+k H\left(Y_{2^{(1)}}[i] \mid V_{2^{(1)}}[i] V_{3^{(1)}}[i]\right) \\
& +\sum_{i=1}^{n} k H\left(Y_{3^{(1)}}[i] \mid V_{3^{(1)}}[i]\right) \\
& +\sum_{i=1}^{n} H\left(Y_{1^{(1)}}[i] \mid V_{1^{(1)}}[i], V_{2^{(1)}}[i], V_{3^{(1)}}[i]\right) \\
& +\sum_{i=1}^{n} k H\left(Y_{2^{(1)}}[i] \mid V_{1^{(1)}}[i], V_{2^{(1)}}[i], V_{3^{(1)}}[i]\right)
\end{aligned}
$$

where step $(a)$ follows from $\left(V_{2^{(2 j+1)}}[i], V_{3^{(j)}}[i]\right)=$ $h_{1}\left(X_{1(2 j)}[i], Y_{1(2 j)}[i]\right), \quad\left(V_{1^{(2 j-1)}}[i], V_{2^{(2 j)}}[i]\right)$ $h_{3}\left(X_{3^{(j)}}[i], Y_{3^{(j)}}[i]\right), \quad\left(V_{1^{(2 j)}}[i], V_{3^{(j)}}[i]\right)$ $h_{2}\left(X_{2^{(2 j+1)}}[i], Y_{2^{(2 j+1)}}[i]\right), \quad\left(V_{2^{(2 j+1)}}[i], V_{3^{(j)}}[i]\right)=$ $h_{1}\left(X_{1^{(2 j+1)}}[i], Y_{1^{(2 j+1)}}[i]\right), \quad V_{3^{(j)}}[i]=g_{3}\left(X_{3^{(j)}}[i]\right)$, $V_{1^{(2 j)}}[i]=g_{1}\left(X_{1^{(2 j)}}[i]\right)$, and $V_{2^{(2 j+1)}}[i]=g_{2}\left(X_{2^{(2 j+1)}}[i]\right)$. Step $(b)$ follows, since the coding schemes of $S_{1^{(j)}} \mathrm{s}, S_{2^{(j)}} \mathrm{s}$, and $S_{3^{(j)}} \mathrm{s}$ are the same respectively, $Y_{1^{(j)}}[i] \mathrm{s}, Y_{2^{(j)}}[i] \mathrm{s}$, and $Y_{3^{(j)}}[i]$ s have the same probability distribution respectively.

\section{Proof of part (b) for bound (41).}

$$
\begin{aligned}
& H\left(Y_{\Omega_{1}^{c}}^{n}\right)+\sum_{l=2}^{5 k+2} H\left(Y_{\Omega_{l}^{c}}^{n} \cap \Omega_{l-1} \mid W_{\mathcal{S} \backslash \Omega_{l}}, Y_{\Omega_{l-1}^{c}}^{n}\right) \\
& \leq \sum_{j} H\left(Y_{2(2 j+1)}^{n} \mid Y_{1(2 j+3)}^{n} X_{1(2 j+3)}^{n}\right) \\
& +\sum_{j} H\left(Y_{1^{(2 j+1)}}^{n} \mid Y_{2^{(2 j+1)}}^{n} X_{2^{(2 j+1)}}^{n}\right) \\
& +\sum_{j} H\left(Y_{3^{(j)}}^{n} \mid Y_{2^{(2 j+1)}}^{n} X_{2^{(2 j+1)}}^{n}\right) \\
& +\sum_{j} H\left(Y_{1(2 j)}^{n} \mid Y_{3(j)}^{n} X_{3(j)}^{n}\right) \\
& +\sum_{j} H\left(Y_{2^{(2 j)}}^{n} \mid Y_{1^{(2 j)}}^{n} X_{1^{(2 j)}}^{n} X_{3^{(j)}}^{n} X_{1^{(2 j+1)}}^{n}\right) \\
& \stackrel{(a)}{\leq} \sum_{j} \sum_{i=1}^{n} H\left(Y_{2^{(2 j+1)}}[i] \mid V_{2^{(2 j+1)}}[i]\right) \\
& +\sum_{j} \sum_{i=1}^{n} H\left(Y_{1(2 j+1)}[i] \mid V_{1^{(2 j+1)}}[i] V_{3^{(j)}}[i]\right) \\
& +\sum_{j} \sum_{i=1}^{n} H\left(Y_{3^{(j)}}[i] \mid V_{2^{(2 j+1)}}[i] V_{3^{(j)}}[i]\right) \\
& +\sum_{j} \sum_{i=1}^{n} H\left(Y_{1^{(2 j)}}[i] \mid V_{1^{(2 j)}}[i] V_{3^{(j)}}[i]\right) \\
& +\sum_{j} \sum_{i=1}^{n} H\left(Y_{2^{(2 j)}}[i] \mid V_{1^{(2 j+1)}}[i], V_{2^{(2 j)}}[i], V_{3^{(j)}}[i]\right) \\
& \stackrel{(b)}{\leq} \sum_{i=1}^{n} k H\left(Y_{2^{(1)}}[i] \mid V_{2^{(1)}}[i]\right)+k H\left(Y_{1^{(1)}}[i] \mid V_{1^{(1)}}[i] V_{3^{(1)}}[i]\right) \\
& +\sum_{i=1}^{n} k H\left(Y_{3^{(1)}}[i] \mid V_{2^{(1)}}[i] V_{3^{(1)}}[i]\right) \\
& +\sum_{i=1}^{n} k H\left(Y_{1^{(1)}}[i] \mid V_{1^{(1)}}[i] V_{3^{(1)}}[i]\right)
\end{aligned}
$$

$$
+\sum_{i=1}^{n} k H\left(Y_{2^{(1)}}[i] \mid V_{1^{(1)}}[i], V_{2^{(1)}}[i], V_{3^{(1)}}[i]\right)
$$

where step $(a)$ follows from $\left(V_{2^{(2 j)}}[i], V_{3^{(j)}}[i]\right)=$ $h_{1}\left(X_{1^{(2 j)}}[i], Y_{1^{(2 j)}}[i]\right), \quad\left(V_{1^{(2 j)}}[i], V_{2^{(2 j+1)}}[i]\right) \quad\left(V_{1(2 j+1)}\right)=$ $h_{3}\left(X_{3^{(j)}}[i], Y_{3^{(j)}}[i]\right), \quad\left(V_{1(2 j+1)}[i], V_{3^{(j)}}[i]\right) \quad=$ $h_{2}\left(X_{2^{(2 j+1)}}[i], Y_{2^{(2 j+1)}}[i]\right), \quad\left(V_{2^{(2 j-1)}}[i], V_{3^{(j)}}[i]\right)=$ $h_{1}\left(X_{1(2 j+1)}[i], Y_{1^{(2 j+1)}}[i]\right), \quad V_{3^{(j)}}[i]=g_{3}\left(X_{3^{(j)}}[i]\right)$, $V_{1^{(2 j+1)}}[i]=g_{1}\left(X_{1^{(2 j+1)}}[i]\right)$, and $V_{2^{(2 j+1)}}[i]=g_{2}\left(X_{2^{(2 j+1)}}[i]\right)$. Step (b) follows, since the coding schemes of $S_{1^{(j)}} \mathrm{S}, S_{2^{(j)}} \mathrm{S}$, and $S_{3^{(j)}} \mathrm{s}$ are the same respectively, $Y_{1^{(j)}}[i] \mathrm{s}, Y_{2^{(j)}}[i] \mathrm{s}$, and $Y_{3^{(j)}}[i] \mathrm{s}$ have the same probability distribution respectively.

Proof of part (b) for bound (42).

$$
\begin{aligned}
& H\left(Y_{\Omega_{1}^{c}}^{n}\right)+\sum_{l=2}^{6} H\left(Y_{\Omega_{l}^{c}}^{n} \cap \Omega_{l-1} \mid W_{\mathcal{S} \backslash \Omega_{l}}, Y_{\Omega_{l-1}^{c}}^{n}\right) \\
& \leq H\left(Y_{1(3)}^{n}\right)+H\left(Y_{3^{(1)}}^{n} \mid Y_{1_{(3)}}^{n} X_{1(3)}^{n}\right) \\
& +H\left(Y_{2(2)}^{n} \mid Y_{1(3)}^{n}, X_{1(3)}^{n}, X_{3(1)}^{n}\right) \\
& +H\left(Y_{1^{(2)}}^{n} \mid Y_{2^{2}}^{n}, X_{2^{2}}^{n}, X_{3^{1}}^{n}\right)+H\left(Y_{2^{(1)}}^{n} \mid Y_{3^{(1)}}^{n}, X_{3^{(1)}}^{n}\right) \\
& +H\left(Y_{1^{(1)}}^{n} \mid Y_{2^{(1)}}^{n}, X_{2^{(1)}}^{n}, X_{3^{(1)}}^{n}\right) \\
& \stackrel{(a)}{\leq} \sum_{i=1}^{n} H\left(Y_{1^{(3)}}[i]\right)+H\left(Y_{3^{(1)}}[i] \mid V_{3^{(1)}}[i]\right) \\
& +\sum_{i=1}^{n} H\left(Y_{2^{(2)}}[i] \mid V_{2^{(2)}}[i] V_{3^{(1)}}[i]\right) \\
& +\sum_{i=1}^{n} H\left(Y_{1^{(2)}}[i] \mid V_{1^{(2)}}[i] V_{2^{(2)}}[i] V_{3^{(1)}}[i]\right) \\
& +\sum_{i=1}^{n} H\left(Y_{2^{(1)}}[i] \mid V_{1^{(1)}}[i] V_{2^{(1)}}[i] V_{3^{(1)}}[i]\right) \\
& +\sum_{i=1}^{n} H\left(Y_{1^{(1)}}[i] \mid V_{1^{(1)}}[i] V_{2^{(1)}}[i] V_{3^{(1)}}[i]\right)+n \epsilon_{n} \\
& \stackrel{(b)}{\leq} \sum_{i=1}^{n} H\left(Y_{1^{(1)}}[i]\right)+H\left(Y_{3^{(1)}}[i] \mid V_{3^{(1)}}[i]\right) \\
& +\sum_{i=1}^{n} H\left(Y_{2^{(1)}}[i] \mid V_{2^{(1)}}[i] V_{3^{(1)}}[i]\right) \\
& +\sum_{i=1}^{n} H\left(Y_{1^{(1)}}[i] \mid V_{1^{(1)}}[i] V_{2^{(1)}}[i] V_{3^{(1)}}[i]\right) \\
& +\sum_{i=1}^{n} H\left(Y_{2^{(1)}}[i] \mid V_{1^{(1)}}[i] V_{2^{(1)}}[i] V_{3^{(1)}}[i]\right) \\
& +\sum_{i=1}^{n} H\left(Y_{1^{(1)}}[i] \mid V_{1^{(1)}}[i] V_{2^{(1)}}[i] V_{3^{(1)}}[i]\right)+n \epsilon_{n}
\end{aligned}
$$

where $(a)$ follows from $V_{2^{(2)}}[i]=g_{2}\left(X_{2^{(2)}}[i]\right)$, $V_{3^{(1)}}[i]=g_{3}\left(X_{3^{(1)}}[i]\right), \quad V_{2^{(1)}}[i]=g_{2}\left(X_{2^{(1)}}[i]\right)$, $\left(V_{2^{(2)}}[i], V_{3^{(1)}}[i]\right) \quad=\quad h_{1}\left(X_{1^{(3)}}[i], Y_{1^{(3)}}[i]\right)$, $\left(V_{1^{(1)}}[i], V_{2^{(1)}}[i]\right)=h_{3}\left(X_{3^{(1)}}[i], Y_{3^{(1)}}[i]\right)$, $\left(V_{1^{(2)}}[i], V_{3^{(1)}}[i]\right)=h_{2}\left(X_{2^{(2)}}[i], Y_{2^{(2)}}[i]\right),\left(V_{1^{(1)}}[i], V_{3^{(1)}}[i]\right)=$ $h_{2}\left(X_{2^{(1)}}[i], Y_{2^{(1)}}[i]\right), \quad$ and $\quad\left(V_{1^{(1)}}[i], V_{3^{(1)}}[i]\right) \quad=$ $h_{2}\left(X_{2^{(1)}}[i], Y_{2^{(1)}}[i]\right)$. Step $(b)$ follows, since the coding schemes of $S_{1^{(j)}}$ and $S_{2^{(j)}}$ are the same respectively, $Y_{1^{(j)}}[i] \mathrm{s}$ and $Y_{2^{(j)}}[i] \mathrm{s}$ have the same probability distribution respectively. 
Proof of part (b) for bound (43).

$$
\begin{aligned}
& H\left(Y_{\Omega_{1}^{c}}^{n}\right)+\sum_{l=2}^{6} H\left(Y_{\Omega_{l}^{c}}^{n} \cap \Omega_{l-1} \mid W_{\mathcal{S} \backslash \Omega_{l}}, Y_{\Omega_{l-1}^{c}}^{n}\right) \\
& \leq H\left(Y_{1^{(3)}}^{n}\right)+H\left(Y_{3^{(1)}}^{n} \mid Y_{1^{(3)}}^{n}, X_{1^{(3)}}^{n}\right) \\
& +H\left(Y_{2^{(2)}}^{n} \mid Y_{1^{(3)}}^{n}, X_{1^{(3)}}^{n}, X_{3^{(1)}}^{n}\right) \\
& +H\left(Y_{1^{(2)}}^{n} \mid Y_{2^{2}}^{n}, X_{2^{2}}^{n}, X_{3^{1}}^{n}\right)+H\left(Y_{2^{(1)}}^{n} \mid Y_{3^{(1)}}^{n}, X_{3^{(1)}}^{n}\right) \\
& +H\left(Y_{1^{(1)}}^{n} \mid Y_{2^{(1)}}^{n}, X_{2^{(1)}}^{n}, X_{3^{(1)}}^{n}\right) \\
& \stackrel{(a)}{\leq} \sum_{i=1}^{n} H\left(Y_{1^{(3)}}[i]\right)+H\left(Y_{3^{(1)}}[i] \mid V_{1^{(3)}}[i], V_{3^{(1)}}[i]\right) \\
& +\sum_{i=1}^{n} H\left(Y_{2^{(2)}}[i] \mid V_{2^{(2)}}[i], V_{3^{(1)}}[i]\right) \\
& +\sum_{i=1}^{n} H\left(Y_{1^{(2)}}[i] \mid V_{1^{(2)}}[i], V_{2^{(2)}}[i], V_{3^{(1)}}[i]\right) \\
& +\sum_{i=1}^{n} H\left(Y_{2^{(1)}}[i] \mid V_{2^{(1)}}[i] V_{3^{(1)}}[i]\right) \\
& +\sum_{i=1}^{n} H\left(Y_{1^{(1)}}[i] \mid V_{1^{(1)}}[i], V_{2^{(1)}}[i], V_{3^{(1)}}[i]\right. \\
& \stackrel{(b)}{\leq} \sum_{i=1}^{n} H\left(Y_{1^{(1)}}[i]\right)+H\left(Y_{3^{(1)}}[i] \mid V_{1^{(1)}}[i], V_{3^{(1)}}[i]\right) \\
& +\sum_{i=1}^{n} H\left(Y_{2^{(1)}}[i] \mid V_{2^{(1)}}[i], V_{3^{(1)}}[i]\right) \\
& +\sum_{i=1}^{n} H\left(Y_{1^{(1)}}[i] \mid V_{1^{(1)}}[i], V_{2^{(1)}}[i], V_{3^{(1)}}[i]\right) \\
& +\sum_{i=1}^{n} H\left(Y_{2^{(1)}}[i] \mid V_{2^{(1)}}[i] V_{3^{(1)}}[i]\right) \\
& +\sum_{i=1}^{n} H\left(Y_{1^{(1)}}[i] \mid V_{1^{(1)}}[i], V_{2^{(1)}}[i], V_{3^{(1)}}[i]\right)
\end{aligned}
$$

where $(a)$ follows from $V_{2^{(2)}}[i]=g_{2}\left(X_{2^{(2)}}[i]\right)$, $V_{3^{(1)}}[i]=g_{3}\left(X_{3^{(1)}}[i]\right), \quad V_{2^{(1)}}[i]=g_{2}\left(X_{2^{(1)}}[i]\right)$, $\left(V_{2^{(2)}}[i], V_{3^{(1)}}[i]\right) \quad=\quad h_{1}\left(X_{1^{(3)}}[i], Y_{1^{(3)}}[i]\right)$, $\left(V_{1^{(1)}}[i], V_{2^{(1)}}[i]\right)=h_{3}\left(X_{3^{(1)}}[i], Y_{3^{(1)}}[i]\right)$, $\left(V_{1^{(2)}}[i], V_{3^{(1)}}[i]\right)=h_{2}\left(X_{2^{(2)}}[i], Y_{2^{(2)}}[i]\right),\left(V_{1^{(1)}}[i], V_{3^{(1)}}[i]\right)=$ $h_{2}\left(X_{2^{(1)}}[i], Y_{2^{(1)}}[i]\right), \quad$ and $\quad\left(V_{1^{(3)}}[i], V_{2^{(1)}}[i]\right) \quad=$ $h_{3}\left(X_{3^{(1)}}[i], Y_{3^{(1)}}[i]\right)$. Step (b) follows, since the coding schemes of $S_{1^{(j)}}$ and $S_{2^{(j)}}$ are the same respectively, $Y_{1^{(j)}}[i] \mathrm{s}$ and $Y_{2^{(j)}}[i] \mathrm{s}$ have the same probability distribution respectively.

Proof of part (b) for bound (44).

$$
\begin{aligned}
& H\left(Y_{\Omega_{1}^{c}}^{n}\right)+\sum_{l=2}^{6 k+2} H\left(Y_{\Omega_{l}^{c} \cap \Omega_{l-1}}^{n} \mid W_{\mathcal{S} \backslash \Omega_{l}}, Y_{\Omega_{l-1}^{c}}^{n}\right) \\
& \leq \sum_{j} H\left(Y_{1^{(3 j+2)}}^{n} \mid Y_{3^{(j+1)}}^{n} X_{3^{(j+1)}}^{n}\right)+H\left(Y_{2^{(2 j+1)}}^{n} \mid Y_{1^{(3 j+2)}}^{n} X_{1^{(3 j+2)}}^{n}\right) \\
& +\sum_{j} H\left(Y_{3^{(j)}}^{n} \mid Y_{1^{(3 j+2)}}^{n} X_{1^{(3 j+2)}}^{n}\right) \\
& +\sum_{j} H\left(Y_{1^{(3 j+1)}}^{n} \mid Y_{3^{(j)}}^{n} X_{3^{(j)}}^{n} Y_{2^{(2 j+1)}}^{n} X_{2^{(2 j+1)}}^{n}\right)
\end{aligned}
$$

$$
\begin{aligned}
& +\sum_{j} H\left(Y_{2^{(2 j)}}^{n} \mid Y_{1^{(3 j+1)}}^{n} X_{1^{(3 j+1)}}^{n} X_{3^{(j)}}^{n}\right) \\
& +\sum_{j} H\left(Y_{1^{(3 j)}}^{n} \mid Y_{2^{(2 j)}}^{n} X_{2^{(2 j)}}^{n} X_{3^{(j)}}^{n}\right) \\
& \stackrel{(a)}{\leq} \sum_{j} \sum_{i=1}^{n} H\left(Y_{1^{(3 j+2)}}[i] \mid V_{1^{(3 j+2)}}[i]\right) \\
& +\sum_{j} \sum_{i=1}^{n} H\left(Y_{2^{(2 j+1)}}[i] \mid V_{2^{(2 j+1)}}[i] V_{3^{(j)}}[i]\right) \\
& +\sum_{j} \sum_{i=1}^{n} H\left(Y_{3^{(j)}}[i] \mid V_{2^{(2 j+1)}}[i] V_{3^{(j)}}[i]\right) \\
& +\sum_{j} \sum_{i=1}^{n} H\left(Y_{1^{(3 j+1)}}[i] \mid V_{1^{(3 j+1)}}[i] V_{2^{(2 j)}}[i] V_{3^{(j)}}[i]\right) \\
& +\sum_{j} \sum_{i=1}^{n} H\left(Y_{2^{(2 j)}}[i] \mid V_{2^{(2 j)}}[i] V_{3^{(j)}}[i]\right) \\
& +\sum_{j} \sum_{i=1}^{n} H\left(Y_{1^{(3 j)}}[i] \mid V_{1^{(3 j)}}[i] V_{2^{(2 j)}}[i] V_{3^{(j)}}[i]\right) \\
& \stackrel{(b)}{\leq} \sum_{i=1}^{n} k H\left(Y_{1^{(1)}}[i] \mid V_{1^{(1)}}[i]\right) \\
& +\sum_{i=1}^{n} k H\left(Y_{3^{(1)}}[i] \mid V_{2^{(1)}}[i] V_{3^{(1)}}[i]\right) \\
& +\sum_{i=1}^{n} 2 k H\left(Y_{1^{(1)}}[i] \mid V_{1^{(1)}}[i] V_{2^{(1)}}[i] V_{3^{(1)}}[i]\right) \\
& +\sum_{i=1}^{n} 2 k H\left(Y_{2^{(1)}}[i] \mid V_{2^{(1)}}[i] V_{3^{(1)}}[i]\right)
\end{aligned}
$$

where step $(a)$ follows from $\left(V_{1^{(3 j)}}[i], V_{3^{(j)}}[i]\right)=$ $h_{2}\left(X_{2^{(2 j)}}[i], Y_{2^{(2 j)}}[i]\right), \quad\left(V_{2^{(2 j)}}[i], V_{3^{(j)}}[i]\right) \quad=$ $h_{1}\left(X_{1(3 j+1)}[i], Y_{1^{(3 j+1)}}[i]\right), \quad\left(V_{1^{(3 j-1)}}[i], V_{2^{(2 j)}}[i]\right)=$ $h_{3}\left(X_{3^{(j)}}[i], Y_{3^{(j)}}[i]\right), \quad\left(V_{1^{(3 j+1)}}[i], V_{3^{(j)}}[i]\right) \quad=$ $h_{2}\left(X_{2^{(2 j+1)}}[i], Y_{2^{(2 j+1)}}[i]\right), \quad\left(V_{2^{(2 j+1)}}[i], V_{3^{(j)}}[i]\right)=$ $h_{1}\left(X_{1^{(3 j+2)}}[i], Y_{1^{(3 j+2)}}[i]\right), \quad V_{3^{(j)}}[i]=g_{3}\left(X_{3^{(j)}}[i]\right)$, and $V_{2^{(2 j)}}[i]=g_{2}\left(X_{2^{(2 j)}}[i]\right)$. Step $(b)$ follows, since the coding schemes of $S_{1^{(j)}} \mathrm{S}, S_{2^{(j)}} \mathrm{S}$, and $S_{3^{(j)}}$ S are the same respectively, $Y_{1^{(j)}}[i] \mathrm{s}, Y_{2^{(j)}}[i] \mathrm{s}$, and $Y_{3^{(j)}}[i] \mathrm{s}$ have the same probability distribution respectively.

\section{Proof of part (b) for bound (45).}

$$
\begin{aligned}
& H\left(Y_{\Omega_{1}^{c}}^{n}\right)+\sum_{l=2}^{6} H\left(Y_{\Omega_{l}^{c} \cap \Omega_{l-1}}^{n} \mid W_{\mathcal{S} \backslash \Omega_{l}}, Y_{\Omega_{l-1}^{c}}^{n}\right) \\
& \leq H\left(Y_{2^{(2)}}^{n}\right)+H\left(Y_{1^{(3)}}^{n} \mid Y_{2^{(2)}}^{n}, X_{2^{(2)}}^{n}\right)+H\left(Y_{3^{(1)}}^{n} \mid Y_{1^{(3)}}^{n}, X_{1^{(3)}}^{n}\right) \\
& +H\left(Y_{1^{(2)}}^{n} \mid Y_{3^{1}}^{n}, X_{3^{1}}^{n}, X_{2^{2}}^{n}\right)+H\left(Y_{2^{(1)}}^{n} \mid Y_{3^{(1)}}^{n}, X_{3^{(1)}}^{n}\right) \\
& +H\left(Y_{1^{(1)}}^{n} \mid Y_{2^{(1)}}^{n}, X_{2^{(1)}}^{n}, X_{3^{(1)}}^{n}\right) \\
& \stackrel{(a)}{\leq} \sum_{i=1}^{n} H\left(Y_{2^{(2)}}[i]\right)+H\left(Y_{1^{(3)}}[i] \mid V_{1^{(3)}}[i], V_{2^{(2)}}[i] V_{3^{(1)}}[i]\right) \\
& +\sum_{i=1}^{n} H\left(Y_{3^{(1)}}[i] \mid V_{3^{(1)}}[i]\right)+H\left(Y_{1^{(2)}}[i] \mid V_{1^{(2)}}[i] V_{2^{(2)}}[i] V_{3^{(1)}}[i]\right) \\
& +\sum_{i=1}^{n} H\left(Y_{2^{(1)}}[i] \mid V_{2^{(1)}}[i] V_{3^{(1)}}[i]\right)+H\left(Y_{1^{(1)}}[i] \mid V_{1^{(1)}}[i] V_{2^{(1)}}[i] V_{3^{(1)}}[i]\right)
\end{aligned}
$$




$$
\begin{aligned}
& \stackrel{(b)}{\leq} \sum_{i=1}^{n} H\left(Y_{2^{(1)}}[i]\right)+H\left(Y_{1^{(1)}}[i] \mid V_{1^{(1)}}[i] V_{2^{(1)}}[i] V_{3^{(1)}}[i]\right) \\
& +\sum_{i=1}^{n} H\left(Y_{3^{(1)}}[i] \mid V_{3^{(1)}}[i]\right)+H\left(Y_{1^{(1)}}[i] \mid V_{1^{(1)}}[i] V_{2^{(1)}}[i] V_{3^{(1)}}[i]\right) \\
& +\sum_{i=1}^{n} H\left(Y_{2^{(1)}}[i] \mid V_{2^{(1)}}[i] V_{3^{(1)}}[i]\right)+H\left(Y_{1^{(1)}}[i] \mid V_{1^{(1)}}[i] V_{2^{(1)}}[i] V_{3^{(1)}}[i]\right)
\end{aligned}
$$

where $(a)$ follows from $V_{2^{(2)}}[i]=g_{2}\left(X_{2^{(2)}}[i]\right)$, $V_{3^{(1)}}[i]=g_{3}\left(X_{3^{(1)}}[i]\right), \quad V_{2^{(1)}}[i]=g_{2}\left(X_{2^{(1)}}[i]\right)$, $\left(V_{1(1)}[i], V_{3(1)}[i]\right) \quad h_{2}\left(X_{2^{(1)}}[i], Y_{2^{(1)}}[i]\right)$, $\left(V_{1^{(2)}}[i], V_{2^{(1)}}[i]\right)=h_{3}\left(X_{3^{(1)}}[i], Y_{3^{(1)}}[i]\right),\left(V_{2^{(2)}}[i], V_{3^{(1)}}[i]\right)=$ $h_{1}\left(X_{1^{(3)}}[i], Y_{1(3)}[i]\right)$, and $\left(V_{1^{(3)}}[i], V_{3^{(1)}}[i]\right)=$ $h_{2}\left(X_{2^{(2)}}[i], Y_{2^{(2)}}[i]\right)$. Step $(b)$ follows, since the coding schemes of $S_{1^{(j)}}$ and $S_{2^{(j)}}$ are the same respectively, $Y_{1^{(j)}}[i] \mathrm{s}$ and $Y_{2^{(j)}}[i] \mathrm{s}$ have the same probability distribution respectively.

\section{Proof of part (b) for bound (46).}

$$
\begin{aligned}
& H\left(Y_{\Omega_{1}^{c}}^{n}\right)+\sum_{l=2}^{7} H\left(Y_{\Omega_{l}^{c}}^{n} \cap \Omega_{l-1} \mid W_{\mathcal{S} \backslash \Omega_{l}}, Y_{\Omega_{l-1}^{c}}^{n}\right) \\
& \leq H\left(Y_{1^{(4)}}^{n}\right)+H\left(Y_{2^{(2)}}^{n} \mid Y_{1^{(4)}}^{n}, X_{1^{(4)}}^{n}\right)+H\left(Y_{1^{(3)}}^{n} \mid Y_{2^{(2)}}^{n}, X_{2^{(2)}}^{n}\right) \\
& +H\left(Y_{3^{(1)}}^{n} \mid Y_{1^{3}}^{n}, X_{1^{3}}^{n}\right)+H\left(Y_{1^{(2)}}^{n} \mid Y_{3^{(1)}}^{n}, X_{\left.2^{(2)}\right)}^{n}, X_{3^{(1)}}^{n}\right) \\
& +H\left(Y_{2^{(1)}}^{n} \mid Y_{3^{(1)}}^{n}, X_{3^{(1)}}^{n}\right)+H\left(Y_{1^{(1)}}^{n} \mid Y_{2^{1}}^{n}, X_{2^{1}}^{n}, X_{3^{(1)}}^{n}\right) \\
& +(a) \sum_{i=1}^{n} H\left(Y_{1^{(4)}}[i]\right)+H\left(Y_{2^{(2)}}[i] \mid V_{2^{(2)}}[i], V_{3^{(1)}}[i]\right) \\
& +\sum_{i=1}^{n} H\left(Y_{1^{(3)}}[i] \mid V_{1^{(3)}}[i], V_{2^{(2)}}[i], V_{3^{(1)}}[i]\right)+H\left(Y_{\left.3^{(1)}\right)}[i] \mid V_{3^{(1)}}[i]\right) \\
& +\sum_{i=1}^{n} H\left(Y_{1^{(2)}}[i] \mid V_{1^{(2)}}[i], V_{2^{(2)}}[i], V_{3^{(1)}}[i]\right)+H\left(Y_{2^{(1)}}[i] \mid V_{2^{(1)}}[i], V_{3^{(1)}}[i]\right) \\
& +\sum_{i=1}^{n} H\left(Y_{1^{(1)}}[i] \mid V_{1^{(1)}}[i], V_{2^{(1)}}[i], V_{3^{(1)}}[i]\right) \\
& \quad \sum_{i=1}^{(b)} H\left(Y_{1^{(1)}}[i]\right)+2 H\left(Y_{2^{(1)}}[i] \mid V_{2^{(1)}}[i], V_{3^{(1)}}[i]\right) \\
& +\sum_{i=1}^{n} 3 H\left(Y_{1^{(1)}}[i] \mid V_{1^{(1)}}[i], V_{2^{(1)}}[i], V_{3^{(1)}}[i]\right)+H\left(Y_{3^{(1)}}[i] \mid V_{3^{(1)}}[i]\right)
\end{aligned}
$$

where $(a)$ follows from $V_{2^{(2)}}[i]=g_{2}\left(X_{2^{(2)}}[i]\right)$, $V_{3^{(1)}}[i]=g_{3}\left(X_{3^{(1)}}[i]\right), \quad V_{2^{(1)}}[i]=g_{2}\left(X_{2^{(1)}}[i]\right)$, $\left(V_{1^{(1)}}[i], V_{3^{(1)}}[i]\right)=h_{2}\left(X_{2^{(1)}}[i], Y_{2^{(1)}}[i]\right)$, $\left(V_{1^{(2)}}[i], V_{2^{(1)}}[i]\right)=h_{3}\left(X_{3^{(1)}}[i], Y_{3^{(1)}}[i]\right)$, $\left(V_{2^{(2)}}[i], V_{3^{(1)}}[i]\right)=h_{1}\left(X_{1^{(3)}}[i], Y_{1^{(3)}}[i]\right),\left(V_{1^{(3)}}[i], V_{3^{(1)}}[i]\right)=$ $h_{2}\left(X_{2^{(2)}}[i], Y_{2^{(2)}}[i]\right), \quad$ and $\quad\left(V_{2^{(2)}}[i], V_{3^{(1)}}[i]\right) \quad=$ $h_{1}\left(X_{1^{(4)}}[i], Y_{1^{(4)}}[i]\right)$. Step (b) follows, since the coding schemes of $S_{1^{(j)}}$ and $S_{2^{(j)}}$ are the same respectively, $Y_{1^{(j)}}[i] \mathrm{s}$ and $Y_{2^{(j)}}[i] \mathrm{s}$ have the same probability distribution respectively.

\section{REFERENCES}

[1] T. M. Cover and J. A. Thomas, Elements of information theory. John Wiley \& Sons, 2012.

[2] L. R. Ford and D. R. Fulkerson, "Maximal fow through a network," Canadian Journal of Mathematics, vol. 8, pp. 399-404, 1956.

[3] R. Ahlswede, N. Cai, S.-Y. Li, and R. Yeung, "Network information flow," Information Theory, IEEE Transactions on, vol. 46, no. 4, pp. 1204-1216, Jul 2000.
[4] A. Avestimehr, S. Diggavi, and D. Tse, "Wireless network information flow: A deterministic approach," Information Theory, IEEE Transactions on, vol. 57, no. 4, pp. 1872-1905, April 2011.

[5] A. Gamal and M. Costa, "The capacity region of a class of deterministic interference channels (corresp.)," Information Theory, IEEE Transactions on, vol. 28, no. 2, pp. 343-346, Mar 1982.

[6] G. Kramer, "Genie-aided outer bounds on the capacity of interference channels," in Information Theory, 2001. Proceedings. 2001 IEEE International Symposium on, 2001, p. 103.

[7] S. U. Kamath, D. N. Tse, and V. Anantharam, "Generalized network sharing outer bound and the two-unicast problem," in Network Coding (NetCod), 2011 International Symposium on. IEEE, 2011, pp. 1-6.

[8] I. Shomorony and A. S. Avestimehr, "A generalized cut-set bound for deterministic multi-flow networks and its applications," Information Theory, IEEE International Symposium on, 2014.

[9] X. Yan, J. Yang, and Z. Zhang, "An outer bound for multisource multisink network coding with minimum cost consideration," IEEE/ACM Transactions on Networking (TON), vol. 14, no. SI, pp. 2373-2385, 2006.

[10] T. Gou and S. A. Jafar, "Sum capacity of a class of symmetric simo gaussian interference channels within," Information Theory, IEEE Transactions on, vol. 57, no. 4, pp. 1932-1958, 2011.

[11] H. Te Sun and K. Kobayashi, "A new achievable rate region for the interference channel," IEEE transactions on information theory, vol. 27, no. 1, pp. 49-60, 1981.

[12] I. Shomorony, "Fundemental of multi-hop multi-flow wireless networks," in Ph.D. Dissertation, ECE, Cornell Univ., August 2014. 